\title{
Supplementary Material for Manuscript
}

The cycloadditions were typically conducted using 1 mmol of the $\beta$ chloroacrylamide dipolarophile as a $0.05 \mathrm{M}$ solution in ether. In some instances, 1-3 mL of acetone was required to solubilise the dipolarophile (see Experimental). Diazoethane and diazomethane were both generated at $-20^{\circ} \mathrm{C}$, and an ethereal solution of the $\beta$ chloroacrylamide was then added to an excess of the dipole ( 7 equivalents) at $-20{ }^{\circ} \mathrm{C}$, the solution was allowed to slowly return to room temperature and was then stirred for 4-6 hours. As phenyldiazomethane was generated at $-50{ }^{\circ} \mathrm{C}$, an ethereal solution of the $\beta$ chloroacrylamide was added to the freshly prepared phenyldiazomethane (10 equivalents) at $-50{ }^{\circ} \mathrm{C}$, the solution was allowed to slowly return to room temperature and was then stirred for 16 hours. All cycloadditions in which trimethylsilyldiazomethane was employed as the dipole were conducted at room temperature, and the reaction time was dependant upon the nature of the dipolarophile; cycloadditions with the sulfoxide derivatives were complete within 6 hours using 5 equivalents of the dipole, while employment of the sulfide derivatives required reaction times of up to 48 hours and up to 15 equivalents of the dipole was necessary. The pyrazoline cycloadducts isolated from the cycloadditions of diazoalkanes to the $\beta$-chloroacrylamides are polar compounds and are very poorly soluble in the majority of organic solvents; in most instances, DMSO- $d_{6}$ was required to record the ${ }^{13} \mathrm{C}$ NMR spectra. This property did, however, facilitate isolation of the cycloadducts as the reaction mixture could simply be filtered at the end of the reaction. The pyrazoline and pyrazole cycloaddducts synthesised during this work are all novel compounds.

The dipolarophilic reactivity of the sulfoxide derivatives of the $\beta$ chloroacrylamides towards diazoethane as the 1,3-dipole was explored first, with initial investigations focusing on the benzylsulfinyl $\beta$-chloroacrylamides, and in particular the $N$-4-fluorophenyl derivative 7. A solution of 7 in ether and acetone (a small quantity of acetone was required to solubilise 7) was added dropwise to an excess of an ethereal solution of diazoethane at $-20{ }^{\circ} \mathrm{C}$. The reaction solution was allowed to return slowly to room temperature and a white solid precipitated out of solution as the reaction proceeded. Following stirring at room temperature for 4 hours, the solvent and excess diazoethane were evaporated under reduced pressure to yield the crude pyrazolines as a $1: 0.22$ mixture of the 2 diastereomers 32a and 32b (Scheme 1). 


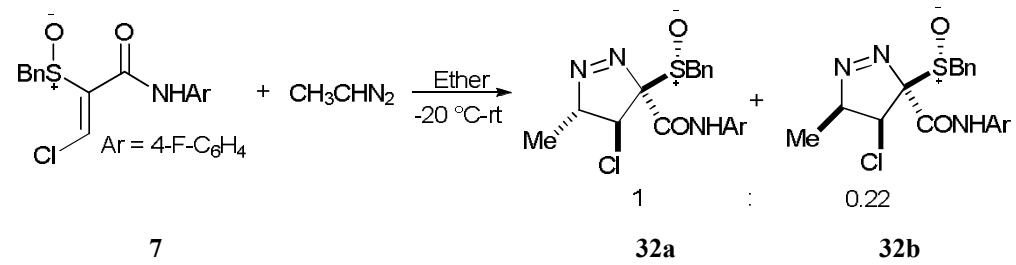

Scheme 1

Purification of the crude cycloadducts proved to be problematic in the early stages. When purification by column chromatography on silica gel was attempted, decomposition of the cycloadducts occurred and a very low recovery of the purified diasteromers resulted. For all subsequent pyrazoline cycloadducts that necessitated chromatographic purification, neutral alumina was used. Hot recrystallisation from solvents such as chloroform and ethyl acetate was also attempted, but the cycloadducts did not survive these conditions and decomposition was still observed. To circumvent decomposition problems, it was decided to isolate the product by filtration, instead of removing the solvent and excess dipole at the end of the reaction by concentration at reduced pressure. The reaction was repeated and again a white solid precipitated out of solution as the reaction proceeded. After stirring at room temperature for 4 hours and then open to the air for a further 0.5 hours to allow excess diazoethane to evaporate, the reaction solution was filtered to give a $1: 0.21$ mixture of the pyrazolines $\mathbf{3 2 a}$ and $\mathbf{3 2} \mathbf{b}$. ${ }^{1} \mathrm{H}$ NMR spectroscopic analysis of the white solid indicated that the cycloadducts were isolated in a pure state, cleaner than by concentration of the reaction mixture, and in a very good yield of $94 \%$.

For the diazoethane cycloadditions, the pyrazolines derived from the $\beta$ chloroacrylamides at the sulfide level of oxidation had greater solubility in most organic solvents than the sulfoxide analogues; it was possible to record the ${ }^{13} \mathrm{C}$ NMR spectra in $\mathrm{CDCl}_{3}$. However, as a result of the greater solubility the isolation of pyrazoline cycloadducts at the sulfide level in pure form (with the exception of 19 and 20) was less trivial than for the corresponding sulfoxide derivatives, which could be recovered by filtration of the product mixture.

The pyrazoles 51, 58-61 are very polar compounds, and use of DMSO- $d_{6}$ is required to record the ${ }^{1} \mathrm{H}$ and ${ }^{13} \mathrm{C}$ NMR spectra.

In cycloadditions of trimethylsilyldiazomethane with the $\alpha$-thio- $\beta$ chloroacrylamides, the consumption of the sulfide was monitored by TLC analysis, and after $5 \mathrm{~h}$ and $24 \mathrm{~h}$ additional portions of five equivalents of trimethylsilyldiazomethane (15 equivalents in total) had to be added to the reaction mixture to force the cycloaddition 
to completion. After stirring for $48 \mathrm{~h}$, there was no evidence of any starting material remaining by TLC analysis.

For cycloadditions of diazomethane with $\alpha$-sulfinyl- $\beta$-chloroacrylamides, a solution of the sulfoxide in ether (a small amount of acetone was also required to solubilise the benzylsulfinyl derivatives) was added dropwise to an excess of an ethereal solution of diazomethane at $-20^{\circ} \mathrm{C}$. The solution was allowed to slowly return to room temperature while stirring for 4 hours. For the benzylsulfinyl and $N$-benzyl- $n$ butylsulfinyl derivatives, a white solid precipitated out of solution as the reaction proceeded and following filtration, each of the pyrazoline cycloadducts 66, 67, 68 and 69 was isolated as a single diastereomer. ${ }^{1} \mathrm{H}$ NMR spectroscopic analysis of the white solid collected by filtration indicated that the cycloadducts were pure.

Examination of the structure of the rearranged pyrazole 63 reveals a number of interesting features. In the solid state, the pyrazole $\mathbf{6 3}$ exists as the tautomer with the carboxamide group at the 3-position of the heterocycle rather than the 5-position (Figure 1). As discussed with the pyrazole 51, it is believed that this tautomer is stabilised by an intramolecular hydrogen bond between the amide $\mathrm{NH}$ and $\mathrm{N}(2)$ of the heterocycle. The 4,5-substituted tautomer may be present in the solution state.
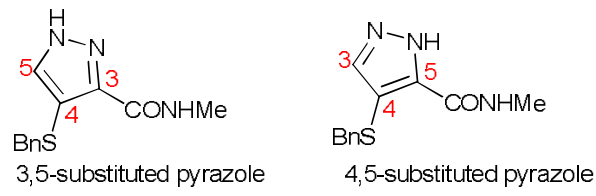

Figure 1

The hydrogen bonding networks are illustrated in Figure 2. There is an intramolecular hydrogen bond between the $\mathrm{NH}$ of the amide and the $\mathrm{N}(2)$ of the heterocycle with a bond length of $2.48 \AA$, an intermolecular hydrogen bond between the $\mathrm{N}(1) \mathrm{H}$ of the heterocycle and the carbonyl group with a bond length of $1.86 \AA$, and an intermolecular hydrogen bond between the $\mathrm{N}(2)$ of the heterocycle and the amide $\mathrm{NH}$ with a bond length of $2.17 \AA$. 


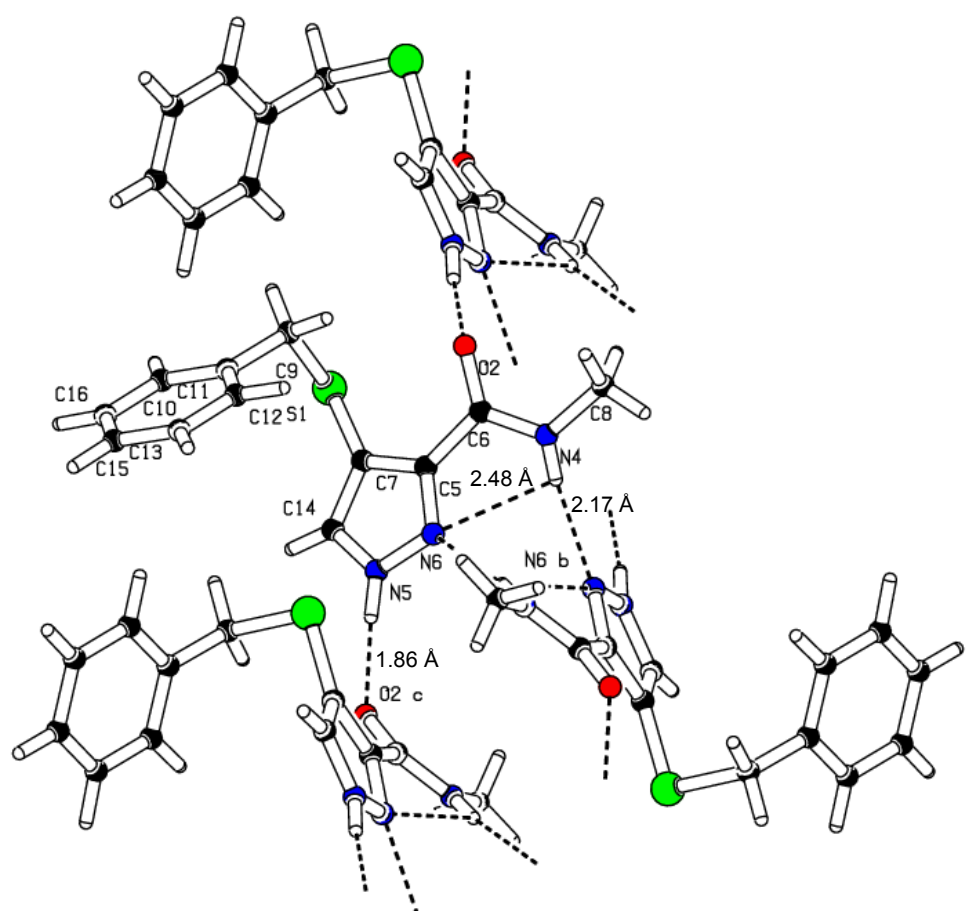

Figure 2

When the cycloaddition of phenyldiazomethane with the $N$-methyl substituted $\beta$ chloroacrylamide 9 was originally carried out, a very poor yield of $10 \%$ was obtained for the pyrazoline $\mathbf{7 7}$ on filtration. The mother liquor was then concentrated to yield a red oil with a complex ${ }^{1} \mathrm{H}$ NMR spectrum, and on purification of this residue by chromatography on silica gel the pyrazole 82 was isolated in $13 \%$ yield. The cycloaddition was then repeated under identical conditions and on filtration of the reaction mixture, the pyrazoline 77 was isolated in $37 \%$ yield on this occasion. The pyrazoline 77 was sufficiently soluble in $\mathrm{CDCl}_{3}$ to record the ${ }^{1} \mathrm{H}$ and ${ }^{13} \mathrm{C} \mathrm{NMR}$ spectrum in this solvent, with no evidence of decomposition. The ${ }^{1} \mathrm{H}$ NMR spectrum of 77 was also recorded in DMSO- $d_{6}$, and approximately $7 \%$ of the sample had decomposed to the pyrazole 82 [characteristic signals for 82 were evident at $\delta_{\mathrm{H}} 2.78 \mathrm{ppm}\left(\mathrm{NHCH}_{3}\right)$ and $\delta_{\mathrm{H}} 8.27 \mathrm{ppm}$ $(\mathrm{NH})]$. The NMR spectra of the pyrazolines in $\mathrm{CDCl}_{3}$ and DMSO- $d_{6}$ suggest different conformations, presumably due to different hydrogen-bonding systems.

The benzenesulfinyl substituted $\beta$-chloroacrylamides 16 and 14 were reacted with freshly prepared phenyldiazomethane, and following stirring at room temperature for 16 hours, the reaction mixtures were concentrated to give the crude products. After purification by column chromatography on silica gel, the pyrazoles $\mathbf{7 9}$ and 80 were isolated in yields of $56 \%$ and $79 \%$ respectively. Precipitates formed as the cycloaddition 
of the benzenesulfinyl derivative $\mathbf{1 5}$ and the $n$-butylsulfinyl derivatives $\mathbf{2 7}$ and $\mathbf{3 1}$ with phenyldiazomethane proceeded, which were isolated by filtration through a sintered glass funnel following stirring at room temperature for 16 hours, to yield the pyrazoles $\mathbf{8 2}, \mathbf{7 9}$ and $\mathbf{8 0}$ as white solids.

\section{Spectroscopic Interpretation of the Cycloadducts}

The pyrazolines 32-37 and 44-50 have a characteristic absorption in the 1532 $1552 \mathrm{~cm}^{-1}$ region of the IR spectra due to stretching of the $\mathrm{N}=\mathrm{N}$ in the pyrazoline ring. ${ }^{1,2}$ The ${ }^{1} \mathrm{H}$ NMR spectra of the pyrazolines 32-37 and 44-50 show some very interesting patterns. The methyl group attached to $\mathrm{C}(5)$ appears as a doublet at $\delta_{\mathrm{H}} 1.46-1.66 \mathrm{ppm}$ for the major diastereomer, whereas the corresponding signal appears further downfield for the minor diastereomer at $\delta_{\mathrm{H}} 1.75-1.87 \mathrm{ppm}$. Another feature in each instance is that for the minor diastereomer, the signal in the ${ }^{1} \mathrm{H}$ NMR spectra for the proton attached to $\mathrm{C}(5)$ appears further upfield than that of the proton attached to $\mathrm{C}(4)$, whereas for the major diastereomer the signal for the proton attached to $\mathrm{C}(5)$ appears further downfield than the signal for the $\mathrm{C}(4)$ proton (for example, see Figure 3).

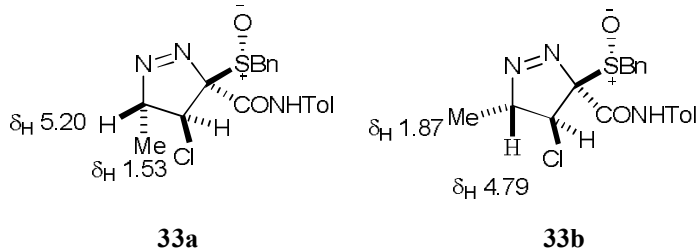

Figure 3

The regiochemistry of the 1,3-dipolar cycloadditions with diazoethane was confirmed by the appearance of the doublet of quartets for the proton attached to $\mathrm{C}(5)$ in the ${ }^{1} \mathrm{H}$ NMR spectra due to coupling to the methyl group at the 5-position and the proton attached to the carbon at the 4-position, and a doublet for the proton attached to $\mathrm{C}(4)$. If the opposite regioisomer was formed, then the signal for $\mathrm{C}(5) \mathrm{H}$ would appear as a quartet due to coupling to the methyl group only and the signal for $\mathrm{C}(3) \mathrm{HCl}$ would be evident as a singlet (Figure 4). 

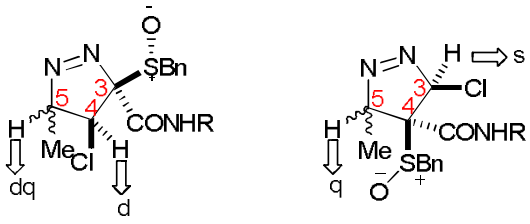

Figure 4

The endo/exo assignment of the diazoethane derived pyrazoline cycloadducts was confirmed by NOE experiments at $600 \mathrm{MHz}$ on the $N$-tolyl substituted pyrazoline 33. For the minor diastereomer 33b, irradiation of $\mathrm{C}(5) \mathrm{CH}_{3}$ at $1.86 \mathrm{ppm}$ resulted in an enhancement of $\mathrm{C}(5) H$ but not $\mathrm{C}(4) H$ [b, Figure 5], irradiation of $\mathrm{C}(5) H$ at $4.78 \mathrm{ppm}$ led to an enhancement of $\mathrm{C}(4) \mathrm{H}$ and $\mathrm{C}(5) \mathrm{CH}_{3}$ [c, Figure 5], while irradiation of $\mathrm{C}(4) \mathrm{H}$ at $5.32 \mathrm{ppm}$ led to an enhancement of $\mathrm{C}(5) \mathrm{H}$ but not $\mathrm{C}(5) \mathrm{CH}_{3}$ [d, Figure 5]. These results are consistent with the exo-diasteromer.

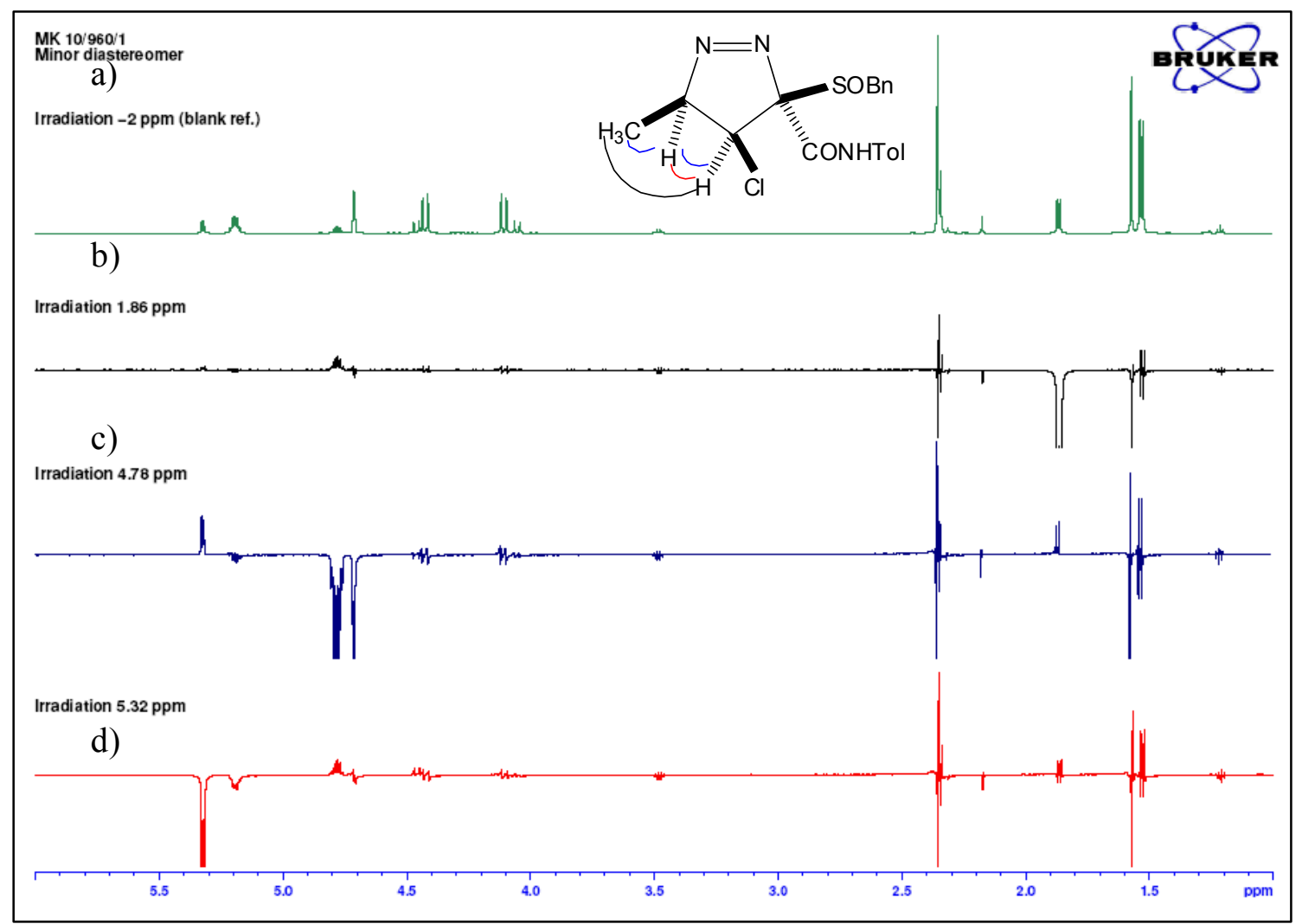

Figure 5 NOE spectra of $33 \boldsymbol{b}$ recorded at $600 \mathrm{MHz}$ in $\mathrm{CDCl}_{3}$ 
The NOE experiment for the major diastereomer 33a supported the assignment, although it was less convincing than the assignment of the minor due to the observation of enhancement between $\mathrm{C}(4) H$ and $\mathrm{C}(5) H$. Irradiation of the methyl group attached to $\mathrm{C}(5)$ at $1.53 \mathrm{ppm}$ led to an enhancement of $\mathrm{C}(4) H$ and $\mathrm{C}(5) H$ [b, Figure 6]. Irradiation of $\mathrm{C}(4) \mathrm{H}$ at $4.71 \mathrm{ppm}$ led to an enhancement of $\mathrm{C}(5) \mathrm{CH}_{3}$ [c, Figure 6], while irradiation of $\mathrm{C}(5) H$ at $5.19 \mathrm{ppm}$ led to an enhancement of $\mathrm{C}(5) \mathrm{CH}_{3}$ [d, Figure 6]. These results are consistent with the endo-diastereomer. However, irradiation of $\mathrm{C}(4) H$ and $\mathrm{C}(5) H$ led to an enhancement of the other signal, indicating the proximity of these protons in space despite their trans orientation.

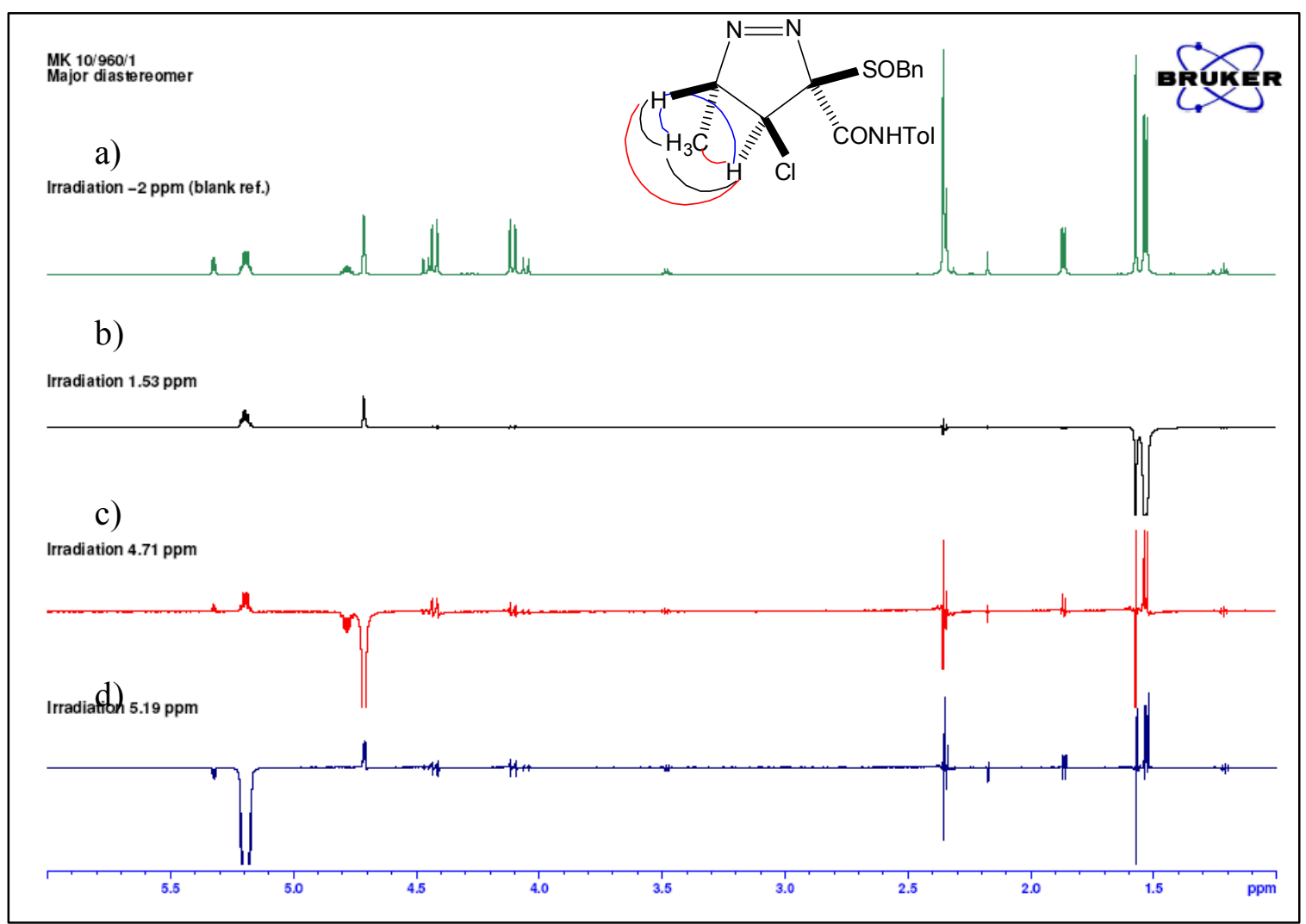

Figure $6 \mathrm{NOE}$ spectra of 33 a recorded at $600 \mathrm{MHz}$ in $\mathrm{CDCl}_{3}$

For the diazoethane derived pyrazoles, a characteristic sharp singlet is seen in the ${ }^{1} \mathrm{H}$ NMR spectra in the region $\delta_{\mathrm{H}} 6.43-6.68 \mathrm{ppm}$ for the proton attached to $\mathrm{C}(4)$, with the corresponding signal in the ${ }^{13} \mathrm{C}$ NMR spectra observed at $\delta_{\mathrm{C}} 104.3-105.7 \mathrm{ppm}$. The 
absorption frequency of the carbonyl stretch in the IR spectra of the pyrazoles is reduced by $15-20 \mathrm{~cm}^{-1}$ when compared to the sulfoxide precursors. ${ }^{3}$ This is due to the delocalisation of the lone pair of the pyrazole nitrogen into the carbonyl group (Figure 7). Two NH stretches (the amide $\mathrm{NH}$ and the $\mathrm{NH}$ of the pyrazole ring) were evident in the IR spectra of the pyrazoles in the region $3110-3390 \mathrm{~cm}^{-1}$.

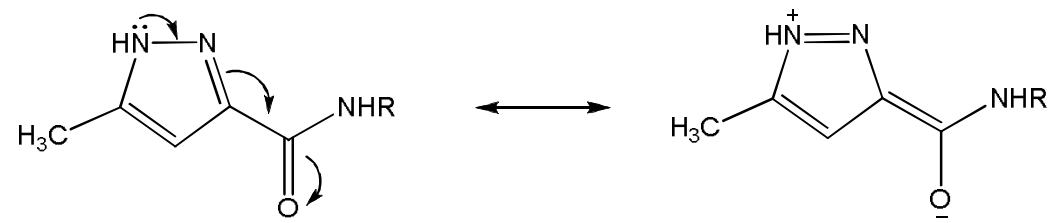

Figure 7

Similar patterns were evident in the ${ }^{1} \mathrm{H}$ NMR spectrum of the pyrazolines 55a and $\mathbf{5 5 b}$ derived from the $\beta$-chloroacrylate 54 as those observed for the $\beta$-chloroacrylamide derived pyrazolines; the methyl group attached to $\mathrm{C}(5)$ appears as a doublet at $\delta_{\mathrm{H}} 1.47$ ppm for the major diastereomer and the corresponding signal for the minor diastereomer appears further downfield at $\delta_{\mathrm{H}} 1.78 \mathrm{ppm}$. For the minor diastereomer, the resonance in the ${ }^{1} \mathrm{H}$ NMR spectrum for the proton attached to $\mathrm{C}(5)$ appears further upfield than that of the proton attached to $\mathrm{C}(4)$, whereas for the major diastereomer the signal for the proton attached to $C(5)$ appears further downfield than the signal for the $C(4)$ proton. The major diastereomer was thus tentatively assigned as endo and the minor diastereomer was assigned as exo.

Another interesting feature of the ${ }^{1} \mathrm{H}$ NMR spectrum of $\mathbf{5 5 a}$ and $\mathbf{5 5 b}$ is the difference in the chemical shift of the methoxy protons; for the minor diastereomer, a $3 \mathrm{H}$ singlet is evident at $\delta_{\mathrm{H}} 3.34 \mathrm{ppm}$, whereas the corresponding signal in the major diastereomer appears much further downfield at $\delta_{\mathrm{H}} 3.62 \mathrm{ppm}$, signifying major conformational differences between the major and minor diastereomers.

Two characteristic broad signals are evident in the ${ }^{1} \mathrm{H}$ NMR spectra of the pyrazoles 51, 58-61 for the proton attached to $C(4)$ and the proton attached to $C(5)$; the $\mathrm{C}(4) \mathrm{H}$ signal appears in the region $\delta_{\mathrm{H}} 6.66-6.79 \mathrm{ppm}$ and the $\mathrm{C}(5) \mathrm{H}$ signal appears at $\delta_{\mathrm{H}}$ 7.74-7.88 ppm. In most cases a broad singlet is observed, but on a few occasions 
coupling between the $\mathrm{C}(4)$ and $\mathrm{C}(5)$ protons leads to fine splitting, and a broad doublet is evident, with a coupling constant of approximately $2 \mathrm{~Hz}$. The analogous signals for $\mathrm{C}(4)$ and $\mathrm{C}(5)$ in the ${ }^{13} \mathrm{C}$ NMR spectra are at $\delta_{\mathrm{C}} 104.7-106.0 \mathrm{ppm}$ for $\mathrm{C}(4)$ and $\delta_{\mathrm{C}} 129.8-131.5$ ppm for $\mathrm{C}(5)$ (Figure 8).

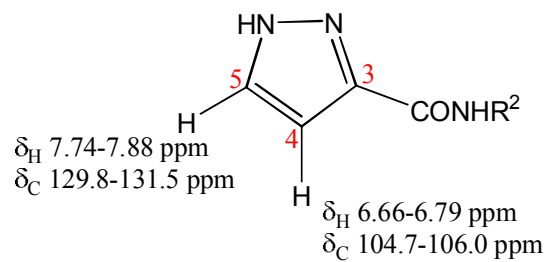

Figure 8

In each instance, the signal for the proton attached to $\mathrm{C}(5)$ is slightly broader in the ${ }^{1} \mathrm{H}$ NMR spectra than the corresponding signal for the proton attached to $\mathrm{C}(4)$. Likewise, the signal for $\mathrm{C}(5)$ is always broader than the signal for $\mathrm{C}(4)$ in the ${ }^{13} \mathrm{C} N \mathrm{NMR}$ spectra; the quadrupolar moment of the adjacent ${ }^{14} \mathrm{~N}$ may be a contributing factor here. Significant broadening of the $C(5)$ signal and the signal for the quaternary carbon at the 3-position of the heterocycle is observed in the ${ }^{13} \mathrm{C}$ NMR spectrum for the $\mathrm{N}$ methylamido pyrazole 61.

In the ${ }^{13} \mathrm{C}$ NMR spectra of the rearranged pyrazoles $62, \mathbf{6 3}$ and $\mathbf{6 5}$, broad signals are observed for the carbons at the 3-position and 5-position of the heterocycle. The quadrupolar moment of ${ }^{14} \mathrm{~N}$ may be contributing to this broadening effect. Interestingly, sharp signals for $\mathrm{C}(3)$ and $\mathrm{C}(5)$ are observed in the ${ }^{13} \mathrm{C}$ NMR spectrum for the pyrazole 64. The ${ }^{13} \mathrm{C}$ NMR spectra of $\mathbf{6 2}, \mathbf{6 3}-\mathbf{6 5}$ also contain a quaternary carbon signal at $\delta_{\mathrm{C}} 107-$ $109 \mathrm{ppm}$, characteristic of the carbon at the 4-position of the heterocycle.

The ${ }^{1} \mathrm{H}$ NMR spectra of $\mathbf{6 4}$ and $\mathbf{6 5}$ were recorded in both $\mathrm{CDCl}_{3}$ and DMSO- $d_{6}$, and the signals for the amide $\mathrm{NH}$ and the $\mathrm{NH}$ of the heterocycle appear further downfield in DMSO- $d_{6}$ (Table 1). Hydrogen bonding may account for these observations, as hydrogen bonding decreases the electron density around the proton and thus moves the proton to higher frequency. In $\mathrm{CDCl}_{3}$, both the amide $\mathrm{NH}$ and $\mathrm{NH}$ of the heterocycle may participate in hydrogen bonding. When recorded in DMSO- $d_{6}$, the solvent may affect the 
intermolecular hydrogen bonding network, and intermolecular hydrogen bonding with DMSO- $d_{6}$ may occur.

Table $1 \delta_{H}$ values for $\mathrm{NH}$ protons of $\mathbf{6 4}$

\begin{tabular}{c|cc} 
& $\boldsymbol{\delta}_{\mathbf{H}}\left(\mathbf{C D C l}_{\mathbf{3}}\right) \mathbf{p p m}$ & $\boldsymbol{\delta}_{\mathbf{H}}\left(\mathbf{D M S O}-\mathbf{d}_{\mathbf{6}}\right) \mathbf{p p m}$ \\
\hline amide $\mathrm{NH}$ & 9.44 & 10.06 \\
$\mathrm{~N}(1) \mathrm{H}$ & 11.33 & 13.75
\end{tabular}

In the ${ }^{13} \mathrm{C}$ NMR spectra of the diazomethane derived pyrazolines 66 and $\mathbf{6 7}$ (recorded in $\mathrm{CDCl}_{3}$ ), the signal for the carbon attached to the chlorine [C(4)] appears at $\delta_{\mathrm{C}} 51-52 \mathrm{ppm}$ and the signal for $\mathrm{C}(5)$ appears at $\delta_{\mathrm{C}} 87-88 \mathrm{ppm}$. It was anticipated that the signal for $\mathrm{C}(4)$ would experience a more significant deshielding effect and that its signal would appear further downfield. The observed effect indicates that the diazo group is more electron withdrawing than chlorine (Figure 9).

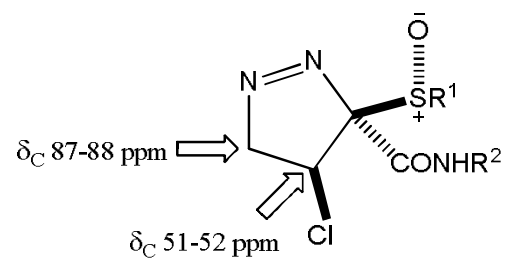

Figure 9

The diazomethane derived pyrazolines 66-69 had a characteristic absorption in the $1540-1550 \mathrm{~cm}^{-1}$ region of the IR spectra due to stretching of the $\mathrm{N}=\mathrm{N}$ in the pyrazoline ring. ${ }^{1,2}$

The electron-withdrawing effect of the diazo group is again evident in the ${ }^{13} \mathrm{C}$ NMR spectra of the pyrazolines $\mathbf{7 0}$ and $\mathbf{7 3}$ (recorded in DMSO- $d_{6}$ ), with the signal for the carbon attached to the chlorine $[\mathrm{C}(4)]$ appearing at $\delta_{\mathrm{C}} 57 \mathrm{ppm}$ and the signal for $\mathrm{C}(5)$ evident at $\delta_{\mathrm{C}} 84 \mathrm{ppm}$.

For the pyrazoline 77, two characteristic doublets were evident in the ${ }^{1} \mathrm{H}$ NMR spectrum at $\delta_{\mathrm{H}} 4.69$ and $5.94 \mathrm{ppm}$ for $\mathrm{C}(4) \mathrm{H}$ and $\mathrm{C}(5) \mathrm{H}$. The deshielding effect of the diazo group on the carbon at the 5-position was again evident in the ${ }^{13} \mathrm{C}$ NMR spectrum, with the signal for the carbon at the 4-position appearing at $\delta_{\mathrm{C}} 59.3 \mathrm{ppm}$ and the carbon at 
the 5-position appearing at $\delta_{\mathrm{C}} 99.8 \mathrm{ppm}$. The structure of the pyrazoline was further confirmed by the presence of an absorption at $1532 \mathrm{~cm}^{-1}$, characteristic of the $\mathrm{N}=\mathrm{N}$ stretch in the pyrazoline system. ${ }^{1,2}$

The structure of the $N$-tolylamido pyrazoline $\mathbf{7 6}$ was confirmed by the presence of two characteristic doublets at $\delta_{\mathrm{H}} 4.83$ and $6.03 \mathrm{ppm}$ in the ${ }^{1} \mathrm{H}$ NMR spectrum when recorded in $\mathrm{CDCl}_{3}$ and by the presence of the $\mathrm{N}=\mathrm{N}$ absorption at $1525 \mathrm{~cm}^{-1}$ in the IR spectrum. Due to the poor solubility of 76 in $\mathrm{CDCl}_{3}, \mathrm{DMSO}-d_{6}$ was necessary to record the ${ }^{13} \mathrm{C}$ NMR spectrum. The ${ }^{1} \mathrm{H}$ NMR spectrum of $\mathbf{7 6}$ was also recorded in DMSO- $d_{6}$ prior to the ${ }^{13} \mathrm{C}$ NMR spectrum, and there was some evidence for decomposition to the pyrazole 79, confirmed by the comparison of the additional signals with a sample of $\mathbf{7 9}$ which was independently synthesised later. When the ${ }^{13} \mathrm{C}$ NMR spectrum was recorded in DMSO- $d_{6}$, total decomposition to the pyrazole 79 had occurred.

As the $N$-benzylamido pyrazoline 78 was insoluble in $\mathrm{CDCl}_{3}$, its ${ }^{1} \mathrm{H}$ NMR spectrum was recorded in DMSO- $d_{6}$; approximately $53 \%$ of the sample had decomposed to the corresponding pyrazole 80, confirmed by the agreement of the decomposition product with a genuine sample of the pyrazole 80. It was thus not possible to obtain the ${ }^{13} \mathrm{C}$ NMR spectrum of $\mathbf{7 6}$ or $\mathbf{7 8}$. The IR spectrum of $\mathbf{7 8}$ contained the characteristic $\mathrm{N}=\mathrm{N}$ stretch at $1525 \mathrm{~cm}^{-1}$ and accurate elemental analysis was also obtained, thereby confirming the pyrazoline structure of $\mathbf{7 8}$ prior to dissolution in DMSO- $d_{6}$.

As mentioned above, two distinctive doublets were evident in the ${ }^{1} \mathrm{H}$ NMR spectra of 76-78, at $\delta_{\mathrm{H}} 4.85-5.12 \mathrm{ppm}$ and $\delta_{\mathrm{H}} 5.53-5.59 \mathrm{ppm}$ in DMSO- $d_{6}$ and at $\delta_{\mathrm{H}}$ 4.69-4.83 ppm and $\delta_{\mathrm{H}} 5.94-6.03 \mathrm{ppm}$ in $\mathrm{CDCl}_{3}$. The upfield signal was assigned as the proton on the carbon attached to the chlorine $[\mathrm{C}(4) \mathrm{H}]$ and the downfield signal was assigned as the $\mathrm{C}(5) \mathrm{H}$ proton. The electron-withdrawing effect of the diazo group on the 5-position of the pyrazoline system was seen earlier for the diazomethane derived pyrazolines, and in the phenyldiazomethane derived pyrazolines the $\mathrm{C}(5) \mathrm{H}$ is also benzylic, leading to a further deshielding of this proton. Interestingly, when the ${ }^{1} \mathrm{H}$ NMR spectra were recorded in $\mathrm{CDCl}_{3}$, the two doublets were further apart. Also, the values of the coupling constants between the protons attached to $\mathrm{C}(4)$ and $\mathrm{C}(5)$ are very different in the two solvents; in $\mathrm{CDCl}_{3}$, coupling constant values of $6.3-6.8 \mathrm{~Hz}$ are observed whereas the corresponding values in DMSO- $d_{6}$ are 8.7-9.0 Hz. Evidently, the pyrazolines adopt 
different conformations in $\mathrm{CDCl}_{3}$ and DMSO- $d_{6}$, presumably due to different hydrogen bonding systems.

A distinctive doublet is observed in the ${ }^{1} \mathrm{H}$ NMR spectra of 79, 80, 82 at approximately $\delta_{\mathrm{H}} 7.80 \mathrm{ppm}$ (when recorded in DMSO- $d_{6}$ ), assigned as the ortho-protons on the phenyl ring attached to the 5-position (Figure 10). Tensmeyer and Ainsworth have attributed this ortho-shift phenomenon to the magnetic anisotropy of the neighbouring ring; the ortho-phenyl protons reside preferentially near the plane of the pyrazole ring and are shifted downfield by the magnetic field of the pyrazole ring current. ${ }^{4}$

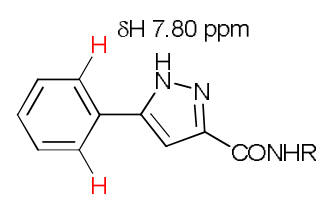

Figure 10

There is a dramatic difference in the position of the NH signal of the carboxamide in the ${ }^{1} \mathrm{H}$ NMR spectra of the pyrazoles $\mathbf{7 9 ,}, \mathbf{8 0}, \mathbf{8 2}$ when recorded in $\mathrm{CDCl}_{3}$ and DMSO$d_{6}$, with the NH signal appearing further downfield by $\sim 1.5 \mathrm{ppm}$ in DMSO- $d_{6}$. This may be due to a change in the nature of the hydrogen bonding network from intramolecular hydrogen bonding in $\mathrm{CDCl}_{3}$ to intermolecular hydrogen bonding with DMSO- $d_{6}$.

The values for the coupling constants of the $\mathrm{C}(4) \mathrm{H}$ and $\mathrm{C}(5) \mathrm{H}$ coupling in $\mathbf{8 6}$ are identical $\left(7.6 \mathrm{~Hz}\right.$ ) in $\mathrm{CDCl}_{3}$ and $\mathrm{DMSO}_{-} d_{6}$ (for the corresponding sulfoxide-derived pyrazolines a larger coupling constant is observed in DMSO- $d_{6}$ ), implying that the pyrazoline adopts similar conformations in $\mathrm{CDCl}_{3}$ and DMSO- $d_{6}$, in contrast to what was seen above for the sulfoxide derived pyrazolines.

\section{Experimental}

$\left(3 R^{*}, 4 R^{*}, 5 R^{*}, S_{\mathrm{S}} *\right)-3-($ Benzylsulfinyl)-4-chloro-4,5-dihydro-5-methyl- $N-(4-$

methylphenyl)-3H-pyrazole-3-carboxamide $33 \mathrm{a}$ and $\left(3 R^{*}, 4 R^{*}, 5 S^{*}, S_{\mathrm{S}}^{*}\right)-3$ (benzylsulfinyl)-4-chloro-4,5-dihydro-5-methyl- $\mathrm{N}$-(4-methylphenyl)-3 $\mathrm{H}$-pyrazole-3carboxamide $33 b$

An excess of an ethereal solution of diazoethane [prepared from $N$-ethyl- $N$-nitrosourea 91 $(0.94 \mathrm{~g}, 8.0 \mathrm{mmol})]$ was added to a solution of $\mathrm{N}$-(4-methylphenyl)-Z-3-chloro-2- 
(benzylsulfinyl)propenamide $8(0.40 \mathrm{~g}, 1.2 \mathrm{mmol})$ in ether $(40 \mathrm{~mL})$ cooled in an ice-salt bath while stirring. The solution was allowed to return slowly to room temperature while stirring for $4 \mathrm{~h}$. A precipitate formed as the reaction progressed. The products were collected by filtration through a sintered glass funnel (grade 3 ) to give 33a and $\mathbf{3 3 b} \mathbf{3} \mathbf{3} \mathbf{a}$ : 33b 1:0.27 by ${ }^{1} \mathrm{H}$ NMR spectroscopy) as a white solid (0.39 g, 84\%), mp 158-160 ${ }^{\circ} \mathrm{C}$; (Found C, 58.34; $\mathrm{H}, 5.26 ; \mathrm{N}, 10.46 ; \mathrm{S}, 8.22, \mathrm{Cl}, 9.70 . \mathrm{C}_{19} \mathrm{H}_{20} \mathrm{ClN}_{3} \mathrm{O}_{2} \mathrm{~S}$ requires $\mathrm{C}, 58.53$; $\mathrm{H}, 5.17$; N , 10.78; S, 8.22, Cl, 9.09\%); $v_{\max } / \mathrm{cm}^{-1}(\mathrm{KBr}) 3292(\mathrm{NH}), 3026(\mathrm{CH}), 1671$ (CO), 1609, $1543(\mathrm{~N}=\mathrm{N}), 1519,1456,1407$;

Major diastereomer 33a: $\delta_{\mathrm{H}}\left(300 \mathrm{MHz}, \mathrm{CDCl}_{3}\right) 1.53\left[3 \mathrm{H}, \mathrm{d}, J 7.5, \mathrm{C}(5) \mathrm{CH} H_{3}\right], 2.35(3 \mathrm{H}, \mathrm{s}$, $\left.\mathrm{ArCH}_{3}\right), 4.11\left(1 \mathrm{H}, \mathrm{d}, \mathrm{A}\right.$ of $\mathrm{AB}$ system, $\left.J_{\mathrm{AB}} 12.7, \mathrm{SCH}_{2}\right), 4.43\left(1 \mathrm{H}, \mathrm{d}, \mathrm{B}\right.$ of AB system, $J_{\mathrm{AB}}$ 12.7, $\left.\mathrm{SCH}_{2}\right), 4.71[1 \mathrm{H}, \mathrm{d}, J 4.0, \mathrm{C}(4) H], 5.20[1 \mathrm{H}, \mathrm{dq}, J 7.5,4.0, \mathrm{C}(5) H], 7.13-7.55(9 \mathrm{H}$, $\mathrm{m}, \operatorname{Ar} H)^{*}, 8.83(1 \mathrm{H}$, br s, $\mathrm{NH})$.

$\delta_{\mathrm{C}}\left(75.5 \mathrm{MHz}, \mathrm{DMSO}-d_{6}\right)$ (signals for major diastereomer 33a only detected) 15.8 $\left[\mathrm{C}(5) \mathrm{CH}_{3}\right], 20.9\left(\mathrm{ArCH}_{3}\right), 54.2\left(\mathrm{SCH}_{2}\right), 61.4[C(5) \mathrm{H}], 90.0[C(4) \mathrm{H}], 107.2[C(3)], 122.0$ (aromatic $C H$ ), 128.7 (aromatic $C H$ or aromatic $C), 129.2,129.4,130.9(3 \times$ aromatic $C \mathrm{H}$ ), 131.3 (aromatic $C \mathrm{H}$ or aromatic $C$ ), 134.5, 135.1 (aromatic $C$ ), $162.3(\mathrm{CO}$ );

Minor diastereomer 33b: $\delta_{\mathrm{H}}\left(300 \mathrm{MHz}, \mathrm{CDCl}_{3}\right) 1.87\left[3 \mathrm{H}, \mathrm{d}, J 7.3, \mathrm{C}(5) \mathrm{CH} H_{3}\right], 2.34(3 \mathrm{H}, \mathrm{s}$, $\left.\mathrm{ArCH}_{3}\right), 4.05\left(1 \mathrm{H}, \mathrm{d}, \mathrm{A}\right.$ of $\mathrm{AB}$ system, $\left.J_{\mathrm{AB}} 12.8, \mathrm{SC} H_{2}\right), 4.46\left(1 \mathrm{H}, \mathrm{d}, \mathrm{B}\right.$ of AB system, $J_{\mathrm{AB}}$ 12.8, $\left.\mathrm{SCH}_{2}\right), 4.75-4.82[1 \mathrm{H}, \mathrm{m}, \mathrm{C}(5) H], 5.32[1 \mathrm{H}, \mathrm{d}, J 5.6, \mathrm{C}(4) H], 7.13-7.55(9 \mathrm{H}, \mathrm{m}$, $\operatorname{Ar} H)^{*}, 9.06(1 \mathrm{H}$, br s, $\mathrm{NH})$.

*The aromatic signals were indistinguishable for the two diastereomers.

HRMS (ES+): Exact mass calculated for $\mathrm{C}_{19} \mathrm{H}_{21} \mathrm{NO}_{2} \mathrm{~S}^{35} \mathrm{Cl}\left[(\mathrm{M}+\mathrm{H})^{+}-\mathrm{N}_{2}\right], 362.0982$. Found $362.0973 ; \mathrm{m} / \mathrm{z}(\mathrm{ES}+) 392.1\left\{\left[\left(\mathrm{C}_{19} \mathrm{H}_{20} \mathrm{~N}_{3} \mathrm{O}_{2} \mathrm{~S}^{37} \mathrm{Cl}\right)+\mathrm{H}^{+}\right], \quad 6 \%\right\}, \quad 390.1$ $\left\{\left[\left(\mathrm{C}_{19} \mathrm{H}_{20} \mathrm{~N}_{3} \mathrm{O}_{2} \mathrm{~S}^{35} \mathrm{Cl}\right)+\mathrm{H}^{+}\right], \quad 12 \%\right\}, \quad 364.2 \quad\left\{\left[\left(\mathrm{C}_{19} \mathrm{H}_{20} \mathrm{NO}_{2} \mathrm{~S}^{37} \mathrm{Cl}\right)+\mathrm{H}^{+}\right], \quad 20 \%\right\}, \quad 362.2$ $\left\{\left[\left(\mathrm{C}_{19} \mathrm{H}_{20} \mathrm{NO}_{2} \mathrm{~S}^{35} \mathrm{Cl}\right)+\mathrm{H}^{+}\right], 46 \%\right\}, 216(100 \%)$.

$\left(3 R^{*}, 4 R^{*}, 5 R^{*}, S_{\mathrm{S}} *\right)-3-($ Benzylsulfinyl)-4-chloro-4,5-dihydro- $N, 5$-dimethyl-3Hpyrazole-3-carboxamide $34 \mathrm{a}$ and $\left(3 R^{*}, 4 R^{*}, 5 S^{*}, S_{\mathrm{S}} *\right)-3$-(benzylsulfinyl)-4-chloro-4,5dihydro- $N, 5$-dimethyl-3H-pyrazole-3-carboxamide 34b

An excess of an ethereal solution of diazoethane [prepared from $N$-ethyl- $N$-nitrosourea 91 $(0.96 \mathrm{~g}, 8.2 \mathrm{mmol})]$ was added to a solution of $N$-methyl-Z-3-chloro-2- 
(benzylsulfinyl)propenamide $9(0.30 \mathrm{~g}, 1.2 \mathrm{mmol})$ in ether $(30 \mathrm{~mL})$ and acetone $(5 \mathrm{~mL})$ cooled in an ice-salt bath while stirring. The solution was allowed to return slowly to room temperature and while stirring for $4 \mathrm{~h}$. A precipitate formed as the reaction progressed. The products were collected by filtration through a sintered glass funnel (grade 3) to give 34a and 34b (34a:34b 1:0.22 by ${ }^{1} \mathrm{H}$ NMR spectroscopy) as a white solid (0.24 g, 43\%), mp 83-84 ${ }^{\circ} \mathrm{C}$; (Found C, 48.79; H, 5.08; N, 13.14. $\mathrm{C}_{13} \mathrm{H}_{16} \mathrm{ClN}_{3} \mathrm{O}_{2} \mathrm{~S}$ requires C, 49.76; H, 5.14; N, 13.39\%); $v_{\max } / \mathrm{cm}^{-1}(\mathrm{KBr}) 3346(\mathrm{NH}), 3030(\mathrm{CH}), 2976$ $(\mathrm{CH}), 1669(\mathrm{CO}), 1542(\mathrm{~N}=\mathrm{N}$ stretch), $1044(\mathrm{SO})$;

Major diastereomer 34a: $\delta_{\mathrm{H}}\left(300 \mathrm{MHz}, \mathrm{CDCl}_{3}\right) 1.47\left[3 \mathrm{H}, \mathrm{d}, J 7.5, \mathrm{C}(5) \mathrm{CH}_{3}\right], 3.00(3 \mathrm{H}, \mathrm{d}$, $J$ 5.0, $\left.\mathrm{NHCH}_{3}\right), 4.04\left(1 \mathrm{H}, \mathrm{d}, \mathrm{A}\right.$ of AB system, $\left.J_{\mathrm{AB}} 12.8, \mathrm{SCH}_{2}\right), 4.39(1 \mathrm{H}, \mathrm{d}, \mathrm{B}$ of $\mathrm{AB}$ system, $\left.J_{\mathrm{AB}} 12.8, \mathrm{SCH} H_{2}\right), 4.61[1 \mathrm{H}, \mathrm{d}, J 4.0, \mathrm{C}(4) H], 5.13[1 \mathrm{H}, \mathrm{dq}, J 7.4,4.0, \mathrm{C}(5) H]$, $6.91(1 \mathrm{H}$, br s, $\mathrm{N} H), 7.28-7.43(5 \mathrm{H}, \mathrm{m}, \mathrm{Ar} H)^{*} ; \delta_{\mathrm{C}}\left(75.5 \mathrm{MHz}, \mathrm{DMSO}-d_{6}\right) 15.8\left[\mathrm{CH}_{3}\right.$, $\left.\mathrm{C}(5) \mathrm{CH}_{3}\right], 26.6\left(\mathrm{CH}_{3}, \mathrm{NHCH}_{3}\right), 54.2\left(\mathrm{CH}_{2}, \mathrm{SCH}_{2}\right), 61.1[\mathrm{CH}, \mathrm{C}(5) \mathrm{H}], 90.2[\mathrm{CH}, C(4) \mathrm{H}]$, $106.8[\mathrm{C}, C(3)], 128.6,129.1,130.8(3 \times \mathrm{CH}$, aromatic $\mathrm{CH}), 131.4,131.5(2 \times \mathrm{C}$, aromatic $C), 164.0(\mathrm{C}, C \mathrm{O})$.

Minor diastereomer 34b: $\delta_{\mathrm{H}}\left(300 \mathrm{MHz}, \mathrm{CDCl}_{3}\right) 1.84\left[3 \mathrm{H}, \mathrm{d}, J 7.3, \mathrm{C}(5) \mathrm{CH}_{3}\right], 2.92(3 \mathrm{H}$, d, $\left.J 4.9, \mathrm{NHCH}_{3}\right), 3.98\left(1 \mathrm{H}, \mathrm{d}, \mathrm{A}\right.$ of AB system, $\left.J_{\mathrm{AB}} 12.9, \mathrm{SCH} H_{2}\right), 4.44(1 \mathrm{H}, \mathrm{d}, \mathrm{B}$ of $\mathrm{AB}$ system, $\left.J_{\mathrm{AB}} 12.9, \mathrm{SCH}_{2}\right), 4.60-4.70[1 \mathrm{H}, \mathrm{m}, \mathrm{C}(5) H], 5.25[1 \mathrm{H}, \mathrm{d}, J 5.4, \mathrm{C}(4) H], 6.96(1 \mathrm{H}$, br s, NH), 7.28-7.43 (5H, m, ArH)*; $\delta_{\mathrm{C}}\left(75.5 \mathrm{MHz}, \mathrm{DMSO}-d_{6}\right) 14.3\left[\mathrm{CH}_{3}, \mathrm{C}(5) \mathrm{CH}_{3}\right]$, $26.8\left(\mathrm{CH}_{3}, \mathrm{NHCH}_{3}\right), 55.3\left(\mathrm{CH}_{2}, \mathrm{SCH}_{2}\right), 59.3[\mathrm{CH}, \mathrm{C}(5) \mathrm{H}], 86.5[\mathrm{CH}, \mathrm{C}(4) \mathrm{H}], 109.9[\mathrm{C}$, $C(3)$ ], $162.6(\mathrm{C}, C \mathrm{C})$ (the aromatic signals were not observed for the minor diastereomer).

HRMS (ES+): Exact mass calculated for $\mathrm{C}_{13} \mathrm{H}_{17} \mathrm{NO}_{2} \mathrm{~S}^{35} \mathrm{Cl}\left[(\mathrm{M}+\mathrm{H})^{+}-\mathrm{N}_{2}\right], 286.0669$. Found 286.0675; m/z (ES+) $316.1 \quad\left\{\left[\left(\mathrm{C}_{13} \mathrm{H}_{16} \mathrm{~N}_{3} \mathrm{O}_{2} \mathrm{~S}^{37} \mathrm{Cl}\right)+\mathrm{H}^{+}\right], \quad 12 \%\right\}, \quad 314.1$ $\left\{\left[\left(\mathrm{C}_{13} \mathrm{H}_{16} \mathrm{~N}_{3} \mathrm{O}_{2} \mathrm{~S}^{35} \mathrm{Cl}\right)+\mathrm{H}^{+}\right], \quad 26 \%\right\}, \quad 288.1 \quad\left\{\left[\left(\mathrm{C}_{13} \mathrm{H}_{16} \mathrm{NO}_{2} \mathrm{~S}^{37} \mathrm{Cl}\right)+\mathrm{H}^{+}\right], \quad 24 \%\right\}, \quad 366.2$ $\left\{\left[\left(\mathrm{C}_{13} \mathrm{H}_{16} \mathrm{NO}_{2} \mathrm{~S}^{35} \mathrm{Cl}\right)+\mathrm{H}^{+}\right], 58 \%\right\}, 231.2(100 \%)$.

This compound decomposed readily at room temperature to give the pyrazole $\mathbf{4 0}$, with characteristic peaks at $\delta_{\mathrm{H}}\left(300 \mathrm{MHz}, \mathrm{DMSO}-d_{6}\right) 2.25\left[3 \mathrm{H}, \mathrm{s}, \mathrm{C}(5) \mathrm{CH}_{3}\right], 2.73(3 \mathrm{H}, \mathrm{s}$, $\left.\mathrm{NHCH}_{3}\right), 6.40[1 \mathrm{H}, \mathrm{s}, \mathrm{C}(4) H], 8.07(1 \mathrm{H}$, br s, $\mathrm{NH}$ of carboxamide), in agreement with data for pure $\mathbf{4 0}$ (see below). 
$\left(3 R^{*}, 4 R *, 5 R *, S_{\mathrm{S}} *\right)-N$-benzyl-3-(benzylsulfinyl)-4-chloro-4,5-dihydro-5-methyl-3Hpyrazole-3-carboxamide $35 \mathrm{a}$ and $\left(3 R^{*}, 4 R^{*}, 5 S^{*}, S_{\mathrm{S}} *\right)-N$-benzyl-3-(benzylsulfinyl)-4chloro-4,5-dihydro-5-methyl-3H-pyrazole-3-carboxamide $35 \mathrm{~b}$

An excess of an ethereal solution of diazoethane [prepared from $N$-ethyl- $N$-nitrosourea 91 $(0.75 \mathrm{~g}, 6.4 \mathrm{mmol})]$ was added to a solution of $N$-benzyl-Z-3-chloro-2(benzylsulfinyl)propenamide $10(0.30 \mathrm{~g}, 0.9 \mathrm{mmol})$ in ether $(30 \mathrm{~mL})$ cooled in an ice-salt bath while stirring. The solution was allowed to return slowly to room temperature while stirring for $4 \mathrm{~h}$. A precipitate formed as the reaction progressed. The products were collected by filtration through a sintered glass funnel (grade 3 ) to give $\mathbf{3 5 a}$ and $\mathbf{3 5} \mathbf{b}$ (35a:35b 1:0.33 by ${ }^{1} \mathrm{H}$ NMR spectroscopy) as a white solid (0.27 g, 77\%), mp 100-101 ${ }^{\circ} \mathrm{C} ; v_{\max } / \mathrm{cm}^{-1}(\mathrm{KBr}) 3350(\mathrm{NH}), 3043(\mathrm{CH}), 2975(\mathrm{CH}), 1666,1537(\mathrm{~N}=\mathrm{N}$ stretch), 1496, $1455,1260,1048$ (SO);

Major diastereomer 35a: $\delta_{\mathrm{H}}\left(300 \mathrm{MHz}, \mathrm{CDCl}_{3}\right) 1.46\left[3 \mathrm{H}, \mathrm{d}, J 7.5, \mathrm{C}(5) \mathrm{CH} H_{3}\right], 3.91(1 \mathrm{H}, \mathrm{d}$, A of AB system, $\left.J_{\mathrm{AB}} 12.8, \mathrm{SCH}_{2}\right), 4.24\left(1 \mathrm{H}, \mathrm{d}, \mathrm{B}\right.$ of $\mathrm{AB}$ system, $\left.J_{\mathrm{AB}} 12.8, \mathrm{SCH}_{2}\right), 4.40-$ $4.71\left[3 \mathrm{H}, \mathrm{m}, \mathrm{NHCH}_{2} \& \mathrm{C}(4) H\right.$ (observed as a doublet at 4.62, J 4.0)], $5.13[1 \mathrm{H}, \mathrm{dq}, J 7.5$, 4.0, $\mathrm{C}(5) H], 7.16-7.50(11 \mathrm{H}, \mathrm{m}, \mathrm{NH} \& \mathrm{Ar} H)^{*} ; \delta_{\mathrm{C}}\left(75.5 \mathrm{MHz}, \mathrm{DMSO}-d_{6}\right) 15.8\left[\mathrm{CH}_{3}\right.$, $\left.\mathrm{C}(5) \mathrm{CH}_{3}\right], 43.3\left(\mathrm{CH}_{2}, \mathrm{NHCH}_{2}\right), 54.4\left(\mathrm{CH}_{2}, \mathrm{SCH}_{2}\right), 61.0[\mathrm{CH}, \mathrm{C}(5) \mathrm{H}], 90.4[\mathrm{CH}, C(4) \mathrm{H}]$, $106.9[\mathrm{C}, C(3)], 163.5(\mathrm{C}, C \mathrm{O})^{\S}$.

Minor diastereomer 35b: $\delta_{\mathrm{H}}\left(300 \mathrm{MHz}, \mathrm{CDCl}_{3}\right) 1.84\left[3 \mathrm{H}, \mathrm{d}, J 7.3, \mathrm{C}(5) \mathrm{CH}_{3}\right], 3.85(1 \mathrm{H}$, d, A of AB system, $\left.J_{\mathrm{AB}} 12.8, \mathrm{SCH}_{2}\right), 4.32\left(1 \mathrm{H}, \mathrm{d}, \mathrm{B}\right.$ of AB system, $\left.J_{\mathrm{AB}} 12.8, \mathrm{SC} H_{2}\right), 4.40-$ $4.71\left[3 \mathrm{H}, \mathrm{m}, \mathrm{NHCH}_{2} \& \mathrm{C}(5) H\right], 5.26[1 \mathrm{H}, \mathrm{d}, J 5.5, \mathrm{C}(4) H], 7.16-7.40(10 \mathrm{H}, \mathrm{m}, \mathrm{ArH})$ *, $7.61(1 \mathrm{H}$, br s, $\mathrm{N} H) ; \delta_{\mathrm{C}}\left(75.5 \mathrm{MHz}, \mathrm{DMSO}-d_{6}\right) 14.2\left[\mathrm{CH}_{3}, \mathrm{C}(5) \mathrm{CH}_{3}\right], 43.5\left(\mathrm{CH}_{2}\right.$, $\left.\mathrm{NHCH}_{2}\right), 55.2\left(\mathrm{CH}_{2}, \mathrm{SCH}_{2}\right), 59.2[\mathrm{CH}, \mathrm{C}(5) \mathrm{H}], 86.7[\mathrm{CH}, \mathrm{C}(4) \mathrm{H}], 109.8[\mathrm{C}, \mathrm{C}(3)], 162.0$ $(\mathrm{C}, \mathrm{CO})^{\S}$.

*The aromatic signals were indistinguishable for the two diastereomers in the ${ }^{1} \mathrm{H}$ NMR. ${ }^{\S}$ The aromatic signals were not distinguishable for the two diastereomers in the ${ }^{13} \mathrm{C}$ NMR and were seen at $\delta_{\mathrm{C}} 127.4,127.5,128.1,128.3,128.7,128.8,129.1,129.2,130.6,130.7$ (aromatic $C \mathrm{H}$ ), 131.3, 138.7 (aromatic $C$ ).

HRMS (ES+): Exact mass calculated for $\mathrm{C}_{19} \mathrm{H}_{21} \mathrm{NO}_{2} \mathrm{~S}^{35} \mathrm{Cl}\left[(\mathrm{M}+\mathrm{H})^{+}-\mathrm{N}_{2}\right], 362.0982$. Found 362.0989; m/z $(\mathrm{ES}+) 392.2\left\{\left[\left(\mathrm{C}_{19} \mathrm{H}_{20} \mathrm{~N}_{3} \mathrm{O}_{2} \mathrm{~S}^{37} \mathrm{Cl}\right)+\mathrm{H}^{+}\right], \quad 14 \%\right\}, \quad 390.2$ 
$\left\{\left[\left(\mathrm{C}_{19} \mathrm{H}_{20} \mathrm{~N}_{3} \mathrm{O}_{2} \mathrm{~S}^{35} \mathrm{Cl}\right)+\mathrm{H}^{+}\right], \quad 32 \%\right\}, \quad 364.2 \quad\left\{\left[\left(\mathrm{C}_{19} \mathrm{H}_{20} \mathrm{NO}_{2} \mathrm{~S}^{37} \mathrm{Cl}\right)+\mathrm{H}^{+}\right], \quad 42 \%\right\}, \quad 362.2$ $\left\{\left[\left(\mathrm{C}_{19} \mathrm{H}_{20} \mathrm{NO}_{2} \mathrm{~S}^{35} \mathrm{Cl}\right)+\mathrm{H}^{+}\right], 100 \%\right\}, 216.2(78 \%)$.

$\left(3 R^{*}, 4 R^{*}, 5 R^{*}, S_{\mathrm{S}}^{*}\right)-3$-(Benzylsulfinyl)- $N$ - $n$-butyl-4-chloro-4,5-dihydro-5-methyl-3Hpyrazole-3-carboxamide 36 a and $\left(3 R^{*}, 4 R^{*}, 5 S^{*}, S_{\mathrm{S}} *\right)-3$-(benzylsulfinyl)- $N$-n-butyl-4chloro-4,5-dihydro-5-methyl-3H-pyrazole-3-carboxamide $36 \mathrm{~b}$

An excess of an ethereal solution of diazoethane [prepared from $N$-ethyl- $N$-nitrosourea 91 (0.90 g, $7.7 \mathrm{mmol})]$ was added to a solution of $N$ - $n$-butyl-Z-3-chloro-2(benzylsulfinyl)propenamide $11(0.33 \mathrm{~g}, 1.1 \mathrm{mmol})$ in ether $(30 \mathrm{~mL})$ cooled in an ice-salt bath while stirring. The solution was allowed to return slowly to room temperature while stirring for $4 \mathrm{~h}$. A precipitate formed as the reaction progressed. The products were collected by filtration through a sintered glass funnel (grade 3 ) to give $\mathbf{3 6 a}$ and $\mathbf{3 6} \mathbf{b}$ (36a:36b 1:0.11 by ${ }^{1} \mathrm{H}$ NMR spectroscopy) as a white solid (0.13 g, 33\%), mp 69-70 ${ }^{\circ} \mathrm{C}$; (Found $\mathrm{C}, 48.15 ; \mathrm{H}, 5.56 ; \mathrm{N}, 10.28 . \mathrm{C}_{16} \mathrm{H}_{22} \mathrm{ClN}_{3} \mathrm{O}_{2} \mathrm{~S}$ requires $\mathrm{C}, 54.00 ; \mathrm{H}, 6.23 ; \mathrm{N}$, $11.81 \%$ )-as this compound decomposed readily at room temperature, it was not possible to get accurate micro analysis; $v_{\max } / \mathrm{cm}^{-1}(\mathrm{KBr}) 3345(\mathrm{NH}), 3029(\mathrm{CH}), 2978(\mathrm{CH}), 1662$ (CO), 1538 ( $\mathrm{N}=\mathrm{N}$ stretch), 1456, 1260, 1228, 1049 (SO);

Major diastereomer 36a: $\delta_{\mathrm{H}}\left(300 \mathrm{MHz}, \mathrm{CDCl}_{3}\right) 0.96\left[3 \mathrm{H}, \mathrm{t}, J\right.$ 7.3, $\left.\mathrm{C}\left(4^{\prime}\right) H\right], 1.32-1.52$ $\left[7 \mathrm{H}, \mathrm{m}, \mathrm{C}\left(3^{\prime}\right) \mathrm{H}_{2}, \mathrm{C}\left(2^{\prime}\right) \mathrm{H}_{2} \& \mathrm{C}(5) \mathrm{CH}_{3} ; \mathrm{C}(5) \mathrm{CH}_{3}\right.$ could be distinguished as a doublet at 1.47 ppm, $J$ 7.5], 3.27-3.55 (2H, sym m, $\left.\mathrm{NHCH}_{2}\right), 4.05\left(1 \mathrm{H}, \mathrm{d}\right.$, A of AB system, $J_{\mathrm{AB}}$ 12.8, $\left.\mathrm{SCH}_{2}\right), 4.38\left(1 \mathrm{H}, \mathrm{d}, \mathrm{B}\right.$ of $\mathrm{AB}$ system, $\left.J_{\mathrm{AB}} 12.8, \mathrm{SCH}_{2}\right), 4.62[1 \mathrm{H}, \mathrm{d}, J 3.8, \mathrm{C}(4) H]$, 5.06-5.20 [1H, m, C(5)H], $6.96\left(1 \mathrm{H}\right.$, br s, NH), 7.18-7.52 (5H, m, ArH); $\delta_{\mathrm{C}}(75.5 \mathrm{MHz}$, DMSO- $d_{6}$ ) (signals for major diastereomer 36a only detected) $14.0\left[C\left(4^{\prime}\right) \mathrm{H}_{3}\right], 15.9$ $\left[C(5) \mathrm{H}_{3}\right], 19.9\left[C\left(3^{\prime}\right) \mathrm{H}_{2}\right], 31.0\left[C\left(2^{\prime}\right) \mathrm{H}_{2}\right], 40.1\left[\mathrm{NHCH}_{2}\right], 54.3\left(\mathrm{SCH}_{2}\right), 61.2[C(5) \mathrm{H}], 90.2$ $[C(4) \mathrm{H}], 107.0[C(3)], 128.7,129.2,130.7,131.5$ (aromatic $C H$ \& aromatic $C$ ), 163.5 $(\mathrm{CO})$.

Minor diastereomer 36b: $\delta_{\mathrm{H}}\left(300 \mathrm{MHz}, \mathrm{CDCl}_{3}\right) 1.84\left[3 \mathrm{H}, \mathrm{d}, J 7.0, \mathrm{C}(5) \mathrm{CH}_{3}\right], 5.25[1 \mathrm{H}$, d, $J 4.7, \mathrm{C}(4) H]$.

HRMS (ES+): Exact mass calculated for $\mathrm{C}_{16} \mathrm{H}_{23} \mathrm{NO}_{2} \mathrm{~S}^{35} \mathrm{Cl}\left[(\mathrm{M}+\mathrm{H})^{+}-\mathrm{N}_{2}\right], 328.1138$. Found $328.1145 ; \mathrm{m} / \mathrm{z}(\mathrm{ES}+) 358.2\left\{\left[\left(\mathrm{C}_{16} \mathrm{H}_{22} \mathrm{~N}_{3} \mathrm{O}_{2} \mathrm{~S}^{37} \mathrm{Cl}\right)+\mathrm{H}^{+}\right], \quad 18 \%\right\}, \quad 356.2$ 
$\left\{\left[\left(\mathrm{C}_{16} \mathrm{H}_{22} \mathrm{~N}_{3} \mathrm{O}_{2} \mathrm{~S}^{35} \mathrm{Cl}\right)+\mathrm{H}^{+}\right], \quad 44 \%\right\}, \quad 330.2 \quad\left\{\left[\left(\mathrm{C}_{16} \mathrm{H}_{22} \mathrm{NO}_{2} \mathrm{~S}^{37} \mathrm{Cl}\right)+\mathrm{H}^{+}\right], \quad 36 \%\right\}, \quad 328.2$ $\left\{\left[\left(\mathrm{C}_{16} \mathrm{H}_{22} \mathrm{NO}_{2} \mathrm{~S}^{35} \mathrm{Cl}\right)+\mathrm{H}^{+}\right], 92 \%\right\}, 182.2(100 \%)$.

$\left(3 R *, 4 R *, 5 R *, S_{\mathrm{S}} *\right)-3-($ Benzylsulfinyl)-4-chloro-4,5-dihydro-5-methyl-3H-pyrazole-3carboxamide 37 a and $\left(3 R^{*}, 4 R *, 5 S^{*}, S_{\mathrm{S}} *\right)-3$-(benzylsulfinyl)-4-chloro-4,5-dihydro-5methyl-3H-pyrazole-3-carboxamide $37 \mathrm{~b}$

An excess of an ethereal solution of diazoethane [prepared from $N$-ethyl- $N$-nitrosourea 91 (1.23 g, 10.5 mmol)] was added to a solution of Z-3-chloro-2(benzylsulfinyl)propenamide $12(0.34 \mathrm{~g}, 1.4 \mathrm{mmol})$ in ether $(30 \mathrm{~mL})$ cooled in an ice-salt bath while stirring. The solution was allowed to return slowly to room temperature while stirring for $4 \mathrm{~h}$. A precipitate formed as the reaction progressed. The products were collected by filtration through a sintered glass funnel (grade 3 ) to give $\mathbf{3 7 a}$ and $\mathbf{3 7} \mathbf{b}$ (37a:37b 1:0.20 by ${ }^{1} \mathrm{H}$ NMR spectroscopy) as a white solid (0.32 g, 75\%), mp 92-93 ${ }^{\circ} \mathrm{C}$; $v_{\max } / \mathrm{cm}^{-1}(\mathrm{KBr}) 3379(\mathrm{NH}), 3028(\mathrm{CH}), 2975(\mathrm{CH}), 1670(\mathrm{CO}), 1538(\mathrm{~N}=\mathrm{N}), 1498$, $1457,1375,1232,1044$ (SO);

Major diastereomer 37a: $\delta_{\mathrm{H}}\left(300 \mathrm{MHz}, \mathrm{CDCl}_{3}\right) 1.50\left[3 \mathrm{H}, \mathrm{d}, J 7.5, \mathrm{C}(5) \mathrm{CH}_{3}\right], 4.14(1 \mathrm{H}, \mathrm{d}$, A of $\mathrm{AB}$ system, $\left.J_{\mathrm{AB}} 12.6, \mathrm{SCH}_{2}\right), 4.42\left(1 \mathrm{H}, \mathrm{d}, \mathrm{B}\right.$ of $\mathrm{AB}$ system, $\left.J_{\mathrm{AB}} 12.8, \mathrm{SCH}_{2}\right), 4.58$ $[1 \mathrm{H}, \mathrm{d}, J 4.0, \mathrm{C}(4) H], 5.09-5.16[1 \mathrm{H}, \mathrm{m}, \mathrm{C}(5) H], 5.89(1 \mathrm{H}, \mathrm{br} \mathrm{s}, \mathrm{N} H), 6.98(1 \mathrm{H}$, br s, $\mathrm{N} H), 7.32-7.48(5 \mathrm{H}, \mathrm{m}, \mathrm{Ar} H)^{*} ; \delta_{\mathrm{C}}\left(75.5 \mathrm{MHz}, \mathrm{DMSO}-d_{6}\right) 15.9\left[C(5) \mathrm{H}_{3}\right], 54.3\left(\mathrm{SCH}_{2}\right)$, $61.2[\mathrm{CH}, C(5) \mathrm{H}], 90.1[\mathrm{CH}, C(4) \mathrm{H}], 106.9[C(3)], 128.7,129.2,130.8(3 \times \mathrm{CH}, 3 \times$ aromatic $\mathrm{CH}$ ), 131.6 (aromatic $C), 165.8(\mathrm{C}, \mathrm{CO})$.

Minor diastereomer 37b: $\delta_{\mathrm{H}}\left(300 \mathrm{MHz}, \mathrm{CDCl}_{3}\right) 1.85\left[3 \mathrm{H}, \mathrm{d}, J 7.3, \mathrm{C}(5) \mathrm{CH}_{3}\right], 4.07(1 \mathrm{H}$, d, A of $\mathrm{AB}$ system, $J_{\mathrm{AB}} 12.8$, one of $\left.\mathrm{SCH}_{2}\right), 4.45\left(1 \mathrm{H}, \mathrm{d}, \mathrm{B}\right.$ of AB system, $J_{\mathrm{AB}} 13.0$, one of $\left.\mathrm{SCH}_{2}\right), 4.61-4.74[1 \mathrm{H}, \mathrm{m}, \mathrm{C}(5) H], 5.21[1 \mathrm{H}, \mathrm{d}, J 5.4, \mathrm{C}(4) H], 5.78(1 \mathrm{H}, \mathrm{br}$ s, NH), 7.15 $(1 \mathrm{H}$, br s, $\mathrm{N} H), 7.32-7.48(5 \mathrm{H}, \mathrm{m}, \mathrm{Ar} H)^{*} ; \delta_{\mathrm{C}}\left(75.5 \mathrm{MHz}, \mathrm{DMSO}-d_{6}\right) 14.3\left[C(5) \mathrm{H}_{3}\right], 55.4$ $\left(\mathrm{SCH}_{2}\right), 59.3[\mathrm{CH}, C(5) \mathrm{H}], 86.5[\mathrm{CH}, \mathrm{C}(4) \mathrm{H}], 164.1(\mathrm{C}, \mathrm{CO})$ (aromatic signals not observed for minor diastereomer).

HRMS (ES+): Exact mass calculated for $\mathrm{C}_{12} \mathrm{H}_{15} \mathrm{NO}_{2} \mathrm{~S}^{35} \mathrm{Cl}\left[(\mathrm{M}+\mathrm{H})^{+}-\mathrm{N}_{2}\right], 272.0512$. Found $272.0525 ; \mathrm{m} / \mathrm{z}(\mathrm{ES}+) 274.1\left\{\left[\left(\mathrm{C}_{12} \mathrm{H}_{14} \mathrm{NO}_{2} \mathrm{~S}^{37} \mathrm{Cl}\right)+\mathrm{H}^{+}\right], \quad 4 \%\right\}, \quad 272.1$ $\left\{\left[\left(\mathrm{C}_{12} \mathrm{H}_{14} \mathrm{NO}_{2} \mathrm{~S}^{35} \mathrm{Cl}\right)+\mathrm{H}^{+}\right], 12 \%\right\}$. 


\section{$N$-Benzyl-5-methyl-1H-pyrazole-3-carboxamide 39}

An excess of an ethereal solution of diazoethane [prepared from $N$-ethyl- $N$-nitrosourea 91 $(0.80 \mathrm{~g}, 6.8 \mathrm{mmol})]$ was added to a solution of $N$-benzyl-Z-3-chloro-2(benzenesulfinyl)propenamide $14(0.31 \mathrm{~g}, 1.0 \mathrm{mmol})$ in ether $(30 \mathrm{~mL})$ and acetone $(10$ $\mathrm{mL}$ ) cooled in an ice-salt bath while stirring. The reaction solution was allowed to return slowly to room temperature while stirring for $6 \mathrm{~h}$. A white solid precipitated out of solution as the reaction progressed. The product was collected by filtration through a sintered glass funnel (grade 3 ) to give the pyrazole 39 as a yellow solid $(0.12 \mathrm{~g}, 58 \%), \mathrm{mp}$ 140-142 ${ }^{\circ} \mathrm{C}$ (As this compound decomposed at room temperature it was not possible to get accurate micro analysis); $v_{\max } / \mathrm{cm}^{-1}(\mathrm{KBr}) 3184(\mathrm{NH}), 3113(\mathrm{NH}), 3029(\mathrm{CH}), 2920$ $(\mathrm{CH}), 1646(\mathrm{CO}), 1562,1492,1434,1293 ; \delta_{\mathrm{H}}\left(300 \mathrm{MHz}, \mathrm{DMSO}-d_{6}\right) 2.26[3 \mathrm{H}, \mathrm{s}$, $\left.\mathrm{C}(5) \mathrm{CH}_{3}\right], 4.42\left(2 \mathrm{H}, \mathrm{d}, J 6.3, \mathrm{NHCH}_{2}\right), 6.46[1 \mathrm{H}, \mathrm{s}, \mathrm{C}(4) H], 7.15-7.44(5 \mathrm{H}, \mathrm{m}, \mathrm{ArH}), 8.72$ $\left(1 \mathrm{H}\right.$, br t, $\mathrm{NH}$ of carboxamide); $\delta_{\mathrm{H}}\left(300 \mathrm{MHz}, \mathrm{CDCl}_{3}\right)(39$ is very poorly soluble in $\left.\mathrm{CDCl}_{3}\right) 2.44\left[3 \mathrm{H}, \mathrm{s}, \mathrm{C}(5) \mathrm{CH}_{3}\right], 4.61\left(2 \mathrm{H}, \mathrm{d}, J 5.9, \mathrm{NHCH}_{2}\right), 6.68[1 \mathrm{H}, \mathrm{s}, \mathrm{C}(4) H], 7.26-$ $7.37(5 \mathrm{H}, \mathrm{m}, \mathrm{Ar} H), 7.64(1 \mathrm{H}$, br t, $\mathrm{N} H$ of carboxamide $)$, a broad signal was also observed at $2.01 \mathrm{ppm}$ (water signal normally observed at $1.60 \mathrm{ppm}) ; \delta_{\mathrm{C}}\left(75.5 \mathrm{MHz}, \mathrm{DMSO}-d_{6}\right)$ $11.1\left[\mathrm{CH}_{3}, \mathrm{C}(5) \mathrm{CH}_{3}\right], 42.2\left(\mathrm{CH}_{2}, \mathrm{NHCH}_{2}\right), 104.5[\mathrm{CH}, \mathrm{C}(4) \mathrm{H}], 127.0,127.6,128.5(3 \times$ $\mathrm{CH}, 3 \times$ aromatic $\mathrm{CH}), 140.2(\mathrm{C}$, aromatic $C), 141.4,145.6[2 \times \mathrm{C}, C(3) \& C(5)], 161.7$ (C, CO); HRMS (ES+): Exact mass calculated for $\mathrm{C}_{12} \mathrm{H}_{14} \mathrm{~N}_{3} \mathrm{O}[\mathrm{M}+\mathrm{H}]^{+}, 216.1137$. Found 216.1136; m/z (ES+) $216.2\left\{\left[\left(\mathrm{C}_{12} \mathrm{H}_{13} \mathrm{~N}_{3} \mathrm{O}\right)+\mathrm{H}^{+}\right], 100 \%\right\}, 90.9(16 \%)$.

\section{N,5-dimethyl-1H-pyrazole-3-carboxamide 40}

An excess of an ethereal solution of diazoethane [prepared from $N$-ethyl- $N$-nitrosourea 91 $(1.01 \mathrm{~g}, 8.7 \mathrm{mmol})]$ was added to a solution of $N$-methyl-Z-3-chloro-2(benzenesulfinyl)propenamide $15(0.30 \mathrm{~g}, 1.2 \mathrm{mmol})$ in ether $(30 \mathrm{~mL})$ and acetone (5 $\mathrm{mL}$ ) cooled in an ice-salt bath while stirring. The reaction solution was allowed to slowly return to room temperature while stirring for $2 \mathrm{~h}$. The solvent was removed by evaporation at reduced pressure to give $\mathbf{4 0}$ as a yellow oil. Recrystallisation from chloroform gave the pyrazole 40 as a white solid $(0.12 \mathrm{~g}, 71 \%), \mathrm{mp} 115-117{ }^{\circ} \mathrm{C}$; $v_{\max } / \mathrm{cm}^{-1}(\mathrm{KBr}) 3430(\mathrm{br}, \mathrm{NH}), 3088(\mathrm{CH}), 2956(\mathrm{CH}), 1649(\mathrm{CO}), 1569,1407,1261 ; \delta_{\mathrm{H}}$ (300 MHz, DMSO-d $\left.d_{6}\right) 2.25\left[3 \mathrm{H}, \mathrm{s}, \mathrm{C}(5) \mathrm{CH}_{3}\right], 2.73\left(3 \mathrm{H}, \mathrm{s}, \mathrm{NHCH}_{3}\right), 6.43[1 \mathrm{H}, \mathrm{s}, \mathrm{C}(4) H]$, 
$8.15(1 \mathrm{H}$, br s, $\mathrm{N} H$ of carboxamide), a broad signal for water was also observed at 5.85 ppm due to exchange with $\mathrm{N}(1) H$ (water signal normally observed at $3.40 \mathrm{ppm}) ; \delta_{\mathrm{C}}(75.5$ $\left.\mathrm{MHz}, \mathrm{DMSO}-d_{6}\right) 11.1\left[\mathrm{CH}_{3}, \mathrm{C}(5) \mathrm{CH}_{3}\right], 25.8\left(\mathrm{CH}_{3}, \mathrm{NHCH}_{3}\right), 104.3[\mathrm{CH}, \mathrm{C}(4) \mathrm{H}], 141.4$, $145.6[2 \times \mathrm{C}, C(3) \& C(5)], 162.0(\mathrm{C}, C \mathrm{O})$; HRMS (ES+): Exact mass calculated for $\mathrm{C}_{6} \mathrm{H}_{10} \mathrm{~N}_{3} \mathrm{O}[\mathrm{M}+\mathrm{H}]^{+}, 140.0824$. Found $140.0829 ; \mathrm{m} / \mathrm{z}(\mathrm{ES}+) 140.1\left\{\left[\left(\mathrm{C}_{6} \mathrm{H}_{9} \mathrm{~N}_{3} \mathrm{O}\right)+\mathrm{H}^{+}\right]\right.$, $100 \%\}$.

Elemental analysis resulted in an underestimation of the carbon, hydrogen and nitrogen content, possibly indicating decomposition or the presence of an inorganic impurity.

\section{5-Methyl- $N$-(4-methylphenyl)-1 $H$-pyrazole-3-carboxamide 41}

An excess of an ethereal solution of diazoethane [prepared from $N$-ethyl- $N$-nitrosourea 91 (0.62 $\mathrm{g}, 5.3 \mathrm{mmol})]$ was added to a solution of $N$-(4-methylphenyl)-Z-3-chloro-2(benzenesulfinyl)propenamide $16(0.24 \mathrm{~g}, 0.8 \mathrm{mmol})$ in ether $(30 \mathrm{~mL})$ and acetone $(5$ $\mathrm{mL}$ ) cooled in an ice-salt bath while stirring. The reaction solution was allowed to return slowly to room temperature while stirring for $4 \mathrm{~h}$. A white solid precipitated out of solution as the reaction progressed. The product was collected by filtration through a sintered glass funnel (grade 3 ) to give the pyrazole $\mathbf{4 1}$ as a low melting white solid (0.11 $\mathrm{g}, 67 \%) ; v_{\max } / \mathrm{cm}^{-1}(\mathrm{KBr}) 3320(\mathrm{NH}), 3135(\mathrm{NH}), 1664(\mathrm{CO}), 1603,1556,1317 ; \delta_{\mathrm{H}}(300$ $\left.\mathrm{MHz}, \mathrm{DMSO}-d_{6}\right) 2.27$ [3H, s, C(5)CH $\mathrm{CH}_{3}$ or $\mathrm{ArCH}_{3}$ ], 2.30 [3H, s, C(5) $\mathrm{CH}_{3}$ or $\mathrm{ArCH}_{3}$ ], 6.55 [1H, s, C(4)H], $7.13(2 \mathrm{H}, \mathrm{d}, J 7.8, \operatorname{Ar} H), 7.68(2 \mathrm{H}, \mathrm{d}, J 7.8, \operatorname{Ar} H), 9.88(1 \mathrm{H}, \mathrm{br}$ s, NH of carboxamide), a broad water signal was also observed at $3.77 \mathrm{ppm}$ due to exchange with $\mathrm{N}(1) H$ (water signal normally observed at $3.40 \mathrm{ppm}) ; \delta_{\mathrm{H}}\left(300 \mathrm{MHz}, \mathrm{CDCl}_{3}\right) 2.33[3 \mathrm{H}, \mathrm{s}$, $\mathrm{C}(5) \mathrm{CH}_{3}$ or $\left.\mathrm{ArCH}_{3}\right], 2.39\left[3 \mathrm{H}, \mathrm{s}, \mathrm{C}(5) \mathrm{CH}_{3}\right.$ or $\left.\mathrm{ArCH}_{3}\right], 6.67[1 \mathrm{H}, \mathrm{s}, \mathrm{C}(4) H], 7.16(2 \mathrm{H}, \mathrm{d}, J$ 8.2, $\operatorname{ArH}), 7.58(2 \mathrm{H}, \mathrm{d}, J$ 8.2, $\mathrm{ArH}), 8.60(1 \mathrm{H}, \mathrm{br} \mathrm{s}, \mathrm{NH}$ of carboxamide), 9.86 [br s, $\mathrm{N}(1) H$, integrates for less than $1 \mathrm{H}] ; \delta_{\mathrm{C}}\left(75.5 \mathrm{MHz}, \mathrm{DMSO}-d_{6}\right) 11.1\left[\mathrm{CH}_{3}, \mathrm{C}(5) \mathrm{CH}_{3}\right], 20.8$ $\left(\mathrm{CH}_{3}, \mathrm{NHCH}_{3}\right), 105.0[\mathrm{CH}, \mathrm{C}(4) \mathrm{H}], 120.4,129.3(2 \times \mathrm{CH}, 2 \times$ aromatic $\mathrm{CH})$, 132.6, $136.6(2 \times \mathrm{C}, 2 \times$ aromatic $C), 141.5,146.2[2 \times \mathrm{C}, C(3) \& C(5)], 160.4(\mathrm{C}, C \mathrm{O}) ; \mathrm{HRMS}$ (ES+): Exact mass calculated for $\mathrm{C}_{12} \mathrm{H}_{14} \mathrm{~N}_{3} \mathrm{O}[\mathrm{M}+\mathrm{H}]^{+}, 216.1137$. Found $216.1138 ; \mathrm{m} / \mathrm{z}$ $(\mathrm{ES}+) 216.2\left\{\left[\left(\mathrm{C}_{12} \mathrm{H}_{13} \mathrm{~N}_{3} \mathrm{O}\right)+\mathrm{H}^{+}\right], 100 \%\right\}$.

Elemental analysis resulted in an underestimation of the carbon, hydrogen and nitrogen content, possibly indicating decomposition or the presence of an inorganic impurity. 


\section{$\mathrm{N}$-n-Butyl-5-methyl-1 $\mathrm{H}$-pyrazole-3-carboxamide 42}

An excess of an ethereal solution of diazoethane [prepared from $N$-ethyl- $N$-nitrosourea 91 $(0.85 \mathrm{~g}, 7.3 \mathrm{mmol})]$ was added to a solution of $N$ - $n$-butyl-Z-3-chloro-2(benzenesulfinyl)propenamide $17(0.30 \mathrm{~g}, 1.0 \mathrm{mmol})$ in ether $(30 \mathrm{~mL})$ cooled in an icesalt bath while stirring. The reaction solution was allowed to return slowly to room temperature while stirring for $6 \mathrm{~h}$. The solvent was removed by evaporation at reduced pressure to give $\mathbf{4 2}$ as a colourless oil. Following purification by column chromatography using hexane: ethyl acetate (gradient elution $20-80 \%$ ethyl acetate) as eluent, the pyrazole 42 was obtained as a white solid (0.05 g, 27\%), mp 122-123 ${ }^{\circ} \mathrm{C}$; (Found C, 59.64; H, 8.40; N, 22.65. $\mathrm{C}_{9} \mathrm{H}_{15} \mathrm{~N}_{3} \mathrm{O}$ requires $\left.\mathrm{C}, 59.64 ; \mathrm{H}, 8.34 ; \mathrm{N}, 23.19 \%\right) ; v_{\max } / \mathrm{cm}^{-1}(\mathrm{KBr}) 3390$ $(\mathrm{NH}), 3186(\mathrm{NH}), 2962(\mathrm{CH}), 1632(\mathrm{CO}), 1589,1459,1407,1260 ; \delta_{\mathrm{H}}\left(300 \mathrm{MHz}, \mathrm{CDCl}_{3}\right)$ $0.94\left[3 \mathrm{H}, \mathrm{t}, J 7.4, \mathrm{C}\left(4^{\prime}\right) H_{3}\right], 1.31-1.49$ [2H, m, C(3') $\left.H_{2}\right], 1.51-1.64\left[2 \mathrm{H}, \mathrm{m}, \mathrm{C}\left(2^{\prime}\right) H_{2}\right], 2.35$ $\left[3 \mathrm{H}, \mathrm{s}, \mathrm{C}(5) \mathrm{CH}_{3}\right], 3.42$ [2H, overlapping dt (appears as a q), $J$ 7.0, 7.0, $\mathrm{NHCH}_{2}$ ], 6.56 $[1 \mathrm{H}, \mathrm{s}, \mathrm{C}(4) H], 6.79(1 \mathrm{H}$, br s, $\mathrm{N} H$ of carboxamide), a broad signal for water was also observed at $\sim 2$ ppm due to exchange with $\mathrm{N}(1) H$ (water signal normally observed at 1.60 ppm); $\delta_{\mathrm{C}}\left(75.5 \mathrm{MHz}, \mathrm{CDCl}_{3}\right) 11.5\left[\mathrm{CH}_{3}, \mathrm{C}(5) \mathrm{CH}_{3}\right], 14.2\left[\mathrm{CH}_{3}, \mathrm{C}\left(4^{\prime}\right) H_{3}\right], 20.5\left[\mathrm{CH}_{2}\right.$, $\left.\mathrm{C}\left(3^{\prime}\right) H_{2}\right], 32.1\left[\mathrm{CH}_{2}, \mathrm{C}\left(2^{\prime}\right) H_{2}\right], 39.3\left(\mathrm{CH}_{2}, \mathrm{NHCH}_{2}\right), 105.2[\mathrm{CH}, \mathrm{C}(4) \mathrm{H}], 141.8,147.1$ [2 $\times \mathrm{C}, C(3) \& C(5)], 162.8(\mathrm{C}, C \mathrm{O})$; HRMS (ES+): Exact mass calculated for $\mathrm{C}_{9} \mathrm{H}_{16} \mathrm{~N}_{3} \mathrm{O}$ $[\mathrm{M}+\mathrm{H}]^{+}, 182.1293$. Found 182.1292; m/z (ES+) $182.2\left\{\left[\left(\mathrm{C}_{9} \mathrm{H}_{15} \mathrm{~N}_{3} \mathrm{O}\right)+\mathrm{H}^{+}\right], 100 \%\right\}$.

\section{5-Methyl- $N$-phenyl-1 $H$-pyrazole-3-carboxamide 43}

An excess of an ethereal solution of diazoethane [prepared from $N$-ethyl- $N$-nitrosourea 91 (1.31 g, $11.2 \mathrm{mmol})]$ was added to a solution of $N$-phenyl-Z-3-chloro-2(benzenesulfinyl)propenamide $18(0.49 \mathrm{~g}, 1.6 \mathrm{mmol})$ in ether $(25 \mathrm{~mL})$ cooled in an icesalt bath while stirring. The reaction solution was allowed to return slowly to room temperature while stirring for $6 \mathrm{~h}$. The solvent was removed by evaporation at reduced pressure to give $\mathbf{4 3}$ as a colourless oil. Following purification by column chromatography using hexane: ethyl acetate (gradient elution $10-80 \%$ ethyl acetate) as eluent, the pyrazole 43 was obtained as a yellow solid $(0.21 \mathrm{~g}, 64 \%), \mathrm{mp} 93-95{ }^{\circ} \mathrm{C} ; v_{\max } / \mathrm{cm}^{-1}(\mathrm{KBr}) 3376$ $(\mathrm{NH}), 3199(\mathrm{NH}), 3137(\mathrm{CH}), 2921(\mathrm{CH}), 1658(\mathrm{CO}), 1597,1540,1440,1317 ; \delta_{\mathrm{H}}(300$ 
$\left.\mathrm{MHz}_{\mathrm{CDCl}}\right) 2.39$ [3H, s, C(5)CH $\left.\mathrm{CH}_{3}\right], 6.68[1 \mathrm{H}, \mathrm{s}, \mathrm{C}(4) H], 7.05-7.19(1 \mathrm{H}, \mathrm{m}, \mathrm{ArH}), 7.30-$ $7.43(2 \mathrm{H}, \mathrm{m}, \mathrm{Ar} H), 7.62-7.74(2 \mathrm{H}, \mathrm{m}, \mathrm{Ar} H), 8.76\left(1 \mathrm{H}, \mathrm{br} \mathrm{s}, \mathrm{N} H\right.$ of carboxamide); $\delta_{\mathrm{C}}(75.5$ $\left.\mathrm{MHz}, \mathrm{CDCl}_{3}\right) 11.4\left[\mathrm{CH}_{3}, \mathrm{C}(5) \mathrm{CH}_{3}\right], 105.7[\mathrm{CH}, \mathrm{C}(4) \mathrm{H}], 120.1,124.5,129.4(3 \times \mathrm{CH}, 3 \times$ aromatic $\mathrm{CH}), 138.2(\mathrm{C}$, aromatic $C), 160.5(\mathrm{C}, \mathrm{CO})^{*}$; HRMS (ES+): Exact mass calculated for $\mathrm{C}_{11} \mathrm{H}_{12} \mathrm{~N}_{3} \mathrm{O}[\mathrm{M}+\mathrm{H}]^{+}$, 202.0980. Found 202.0987; m/z (ES+) 202.2 $\left\{\left[\left(\mathrm{C}_{11} \mathrm{H}_{11} \mathrm{~N}_{3} \mathrm{O}\right)+\mathrm{H}^{+}\right], 100 \%\right\}$.

${ }^{*} \mathrm{C}(3)$ and $\mathrm{C}(5)$ were not detected in the ${ }^{13} \mathrm{C}$ NMR spectrum.

$\left(3 R^{*}, 4 R^{*}, 5 R^{*}\right)-3$-(Benzylthio)-4-chloro- $N$-(4-fluorophenyl)-4,5-dihydro-5-methyl-

$3 H$-pyrazole-3-carboxamide 44 a and $\left(3 R^{*}, 4 R^{*}, 5 S^{*}\right)-3$-(benzylthio)-4-chloro- $N$-(4fluorophenyl)-4,5-dihydro-5-methyl-3 $\mathrm{H}$-pyrazole-3-carboxamide 44b

An excess of an ethereal solution of diazoethane [prepared from $N$-ethyl- $N$-nitrosourea 91 $(0.71 \mathrm{~g}, 6.1 \mathrm{mmol})]$ was added to a solution of $\mathrm{N}$-(4-fluorophenyl)-Z-3-chloro-2(benzylthio)propenamide $19(0.28 \mathrm{~g}, 0.9 \mathrm{mmol})$ in ether $(25 \mathrm{~mL})$ cooled in an ice-salt bath while stirring. The solution was allowed to return slowly to room temperature while stirring for $6 \mathrm{~h}$. A precipitate formed as the reaction progressed. The products were collected by filtration through a sintered glass funnel (grade 3 ) to give $\mathbf{4 4 a}$ and $\mathbf{4 4 b}$ (44a:44b 1:0.08 by ${ }^{1} \mathrm{H}$ NMR spectroscopy) as a white solid (0.24 g, 74\%), mp 220-221 ${ }^{\circ} \mathrm{C} ; v_{\max } / \mathrm{cm}^{-1}(\mathrm{KBr}) 3241(\mathrm{NH}), 1663(\mathrm{CO}), 1526(\mathrm{~N}=\mathrm{N}$ stretch), 1507;

Major diastereomer 44a: $\delta_{\mathrm{H}}\left(300 \mathrm{MHz}, \mathrm{CDCl}_{3}\right) 1.66\left[3 \mathrm{H}, \mathrm{d}, J 7.3, \mathrm{C}(5) \mathrm{CH} \mathrm{H}_{3}\right], 4.04(1 \mathrm{H}, \mathrm{d}$, A of $\mathrm{AB}$ system, $\left.J_{\mathrm{AB}} 12.1, \mathrm{SC} H_{2}\right), 4.10\left(1 \mathrm{H}, \mathrm{d}, \mathrm{B}\right.$ of $\mathrm{AB}$ system, $\left.J_{\mathrm{AB}} 12.2, \mathrm{SCH}_{2}\right), 4.21$ $[1 \mathrm{H}, \mathrm{d}, J \text { 7.4, C(4)H], 4.64-4.78 [1H, m, C(5)H], 6.97-7.09 (2H, m, } \operatorname{Ar} H)^{*}, 7.16-7.52$ $(7 \mathrm{H}, \mathrm{m}, \mathrm{Ar} H)^{*}, 8.64(1 \mathrm{H}$, br s, $\mathrm{NH})$.

*These signals were indistinguishable for the two diastereomers.

$\delta_{\mathrm{C}}\left(75.5 \mathrm{MHz}, \mathrm{CDCl}_{3}\right)$ (signals for major diastereomer $44 \mathrm{a}$ only detected) $13.7\left[\mathrm{CH}_{3}\right.$, $\left.\mathrm{C}(5) \mathrm{CH}_{3}\right], 33.2\left(\mathrm{CH}_{2}, \mathrm{SCH}_{2}\right), 59.5[\mathrm{CH}, \mathrm{C}(5) \mathrm{H}], 89.4[\mathrm{CH}, \mathrm{C}(4) \mathrm{H}], 96.2[\mathrm{C}, \mathrm{C}(3)], 114.0$ $\left[\mathrm{CH}, \mathrm{d},{ }^{2} J_{\mathrm{CF}} 22\right.$, aromatic $\left.C\left(3^{\prime}\right) \mathrm{H}\right], 119.8\left[\mathrm{CH}, \mathrm{d},{ }^{3} J_{\mathrm{CF}} 8\right.$, aromatic $\left.C\left(2^{\prime}\right) \mathrm{H}\right], 126.0,127.0$, 127.3, $(3 \times \mathrm{CH}, 3 \times$ aromatic $\mathrm{CH}), 130.8,133.6(2 \times \mathrm{C}, 2 \times$ aromatic $C), 158.0\left[\mathrm{C}, \mathrm{d},{ }^{1} J_{\mathrm{CF}}\right.$ 245, aromatic $\left.C\left(4^{\prime}\right)\right], 162.5(\mathrm{C}, C \mathrm{O})$. 
Minor diastereomer 44b: $\delta_{\mathrm{H}}\left(300 \mathrm{MHz}, \mathrm{CDCl}_{3}\right) 1.77\left[3 \mathrm{H}, \mathrm{d}, J 7.4, \mathrm{C}(5) \mathrm{CH}_{3}\right], 4.15(2 \mathrm{H}$, d, $\left.J 3.4, \mathrm{SCH}_{2}\right), 4.64-4.78[1 \mathrm{H}, \mathrm{m}, \mathrm{C}(5) H]^{*}, 4.98[1 \mathrm{H}, \mathrm{d}, J 6.5, \mathrm{C}(4) H], 6.97-7.09(2 \mathrm{H}, \mathrm{m}$, $\operatorname{Ar} H)^{*}, 7.16-7.52(7 \mathrm{H}, \mathrm{m}, \operatorname{Ar} H)^{*}, 8.37(1 \mathrm{H}$, br s, NH).

*These signals were indistinguishable for the two diastereomers.

HRMS (ES+): Exact mass calculated for $\mathrm{C}_{18} \mathrm{H}_{18} \mathrm{NOS}^{35} \mathrm{ClF}\left[(\mathrm{M}+\mathrm{H})^{+}-\mathrm{N}_{2}\right], 350.0782$. Found $350.0783 ; \mathrm{m} / \mathrm{z}(\mathrm{ES}+) 352.1\left\{\left[\left(\mathrm{C}_{18} \mathrm{H}_{17} \mathrm{NOS}^{37} \mathrm{ClF}\right)+\mathrm{H}^{+}\right], \quad 12 \%\right\}, \quad 350.2$ $\left\{\left[\left(\mathrm{C}_{18} \mathrm{H}_{17} \mathrm{NOS}^{35} \mathrm{ClF}\right)+\mathrm{H}^{+}\right], 26 \%\right\}$.

Elemental analysis resulted in a significant underestimation of the carbon, hydrogen and nitrogen content, possibly indicating decomposition or the presence of an inorganic impurity.

\section{$\left(3 R^{*}, 4 R *, 5 R *\right)-3-($ Phenylthio)-4-chloro- $N$-(4-fluorophenyl)-4,5-dihydro-5-methyl-}

$3 H$-pyrazole-3-carboxamide 47 a and $\left(3 R^{*}, 4 R^{*}, 5 S^{*}\right)-3$-(phenylthio)-4-chloro- $N$-(4fluorophenyl)-4,5-dihydro-5-methyl-3 $\mathrm{H}$-pyrazole-3-carboxamide $47 \mathrm{~b}$

An excess of an ethereal solution of diazoethane [prepared from $N$-ethyl- $N$-nitrosourea 91 $(0.79 \mathrm{~g}, 6.7 \mathrm{mmol})]$ was added to a solution of $\mathrm{N}$-(4-fluorophenyl)-Z-3-chloro-2(phenylthio)propenamide $22(0.30 \mathrm{~g}, 1.0 \mathrm{mmol})$ in ether $(25 \mathrm{~mL})$ cooled in an ice-salt bath while stirring. The reaction solution was allowed to return slowly to room temperature while stirring for $6 \mathrm{~h}$. The solvent was removed by concentration at reduced pressure to give the crude products $47 \mathbf{a}$ and $\mathbf{4 7} \mathbf{b}\left(\mathbf{4 7 a : 4 7 b} 1: 0.28\right.$ by ${ }^{1} \mathrm{H}$ NMR spectroscopy) as a pale yellow oil. Crystallisation from ether/hexane gave $47 \mathbf{a}$ and $\mathbf{4 7 b}$ (47a:47b 1:0.20 by ${ }^{1} \mathrm{H}$ NMR spectroscopy) as a white solid $(0.26 \mathrm{~g}, 75 \%), \mathrm{mp} 87-88^{\circ} \mathrm{C}$; (Found C, 56.07; H, 4.22; N, 11.51; S, 8.50; Cl, 9.97; F, 5.57. $\mathrm{C}_{17} \mathrm{H}_{15} \mathrm{ClFN}_{3} \mathrm{OS}$ requires C, 56.12; H, 4.16; N, 11.55; S, 8.81; Cl, 9.74; F, 5.22\%); $v_{\max } / \mathrm{cm}^{-1}(\mathrm{KBr}) 3288(\mathrm{NH})$, $3014(\mathrm{CH}), 2980(\mathrm{CH}), 1681(\mathrm{CO}), 1516(\mathrm{~N}=\mathrm{N}$ stretch);

Major diastereomer 47a: $\delta_{\mathrm{H}}\left(300 \mathrm{MHz}, \mathrm{CDCl}_{3}\right) 1.57\left[3 \mathrm{H}, \mathrm{d}, J 7.3, \mathrm{C}(5) \mathrm{CH}_{3}\right], 4.28[1 \mathrm{H}, \mathrm{d}$, $J$ 6.7, C(4)H], 4.57-4.67 [1H, sym m, C(5)H], 6.91-6.97, 7.13-7.18, 7.29-7.48, 7.66-7.85 $(10 \mathrm{H}, \mathrm{m}, \mathrm{ArH} \& \mathrm{NH})^{*} ; \delta_{\mathrm{C}}\left(75.5 \mathrm{MHz}, \mathrm{CDCl}_{3}\right) 16.0\left[\mathrm{CH}_{3}, \mathrm{C}(5) \mathrm{CH}_{3}\right], 60.7[\mathrm{CH}, \mathrm{C}(5) \mathrm{H}]$, $91.6[\mathrm{CH}, C(4) \mathrm{H}], 98.7[\mathrm{C}, C(3)]^{\S}, 116.0\left[\mathrm{CH}, \mathrm{d},{ }^{2} J_{\mathrm{CF}} 23 \text {, aromatic } C\left(3^{\prime}\right) \mathrm{H}\right]^{\S}, 122.1[\mathrm{CH}$, $\mathrm{d},{ }^{3} J_{\mathrm{CF}} 8$, aromatic $\left.C\left(2^{\prime}\right) \mathrm{H}\right]^{\S}, 128.3(\mathrm{C} \text {, aromatic } C)^{\S}, 129.6,130.9(2 \times \mathrm{CH}, 2 \times$ aromatic 
$C \mathrm{H}), 132.7(\mathrm{C} \text {, aromatic } C)^{\S}, 138.0(\mathrm{CH}$, aromatic $C \mathrm{H}), 160.1\left[\mathrm{C}, \mathrm{d},{ }^{1} J_{\mathrm{CF}} 245\right.$, aromatic $\left.C\left(4^{\prime}\right)\right]^{\S}, 164.5(\mathrm{C}, C \mathrm{O})^{\S}$.

Minor diastereomer 47b: $\delta_{\mathrm{H}}\left(300 \mathrm{MHz}, \mathrm{CDCl}_{3}\right) 1.80\left[3 \mathrm{H}, \mathrm{d}, J 7.3, \mathrm{C}(5) \mathrm{CH}_{3}\right], 4.46-4.55$ $[1 \mathrm{H}$, sym m, C $(5) H], 5.06[1 \mathrm{H}, \mathrm{d}, J 5.9, \mathrm{C}(4) H], 6.91-7.85(10 \mathrm{H}, \mathrm{m}, \mathrm{ArH} \& \mathrm{~N} H)^{*} ; \delta_{\mathrm{C}}$ $\left(75.5 \mathrm{MHz}, \mathrm{CDCl}_{3}\right) 14.1\left[\mathrm{CH}_{3}, \mathrm{C}(5) \mathrm{CH}_{3}\right], 61.2[\mathrm{CH}, \mathrm{C}(5) \mathrm{H}], 86.2[\mathrm{CH}, \mathrm{C}(4) \mathrm{H}], 129.7$, $130.7(2 \times \mathrm{CH}, 2 \times$ aromatic $\mathrm{CH}), 137.4(\mathrm{CH}$, aromatic $\mathrm{CH})$.

*These signals were indistinguishable for the two diastereomers.

${ }^{\S}$ The analogous signals were not detected for the minor diastereomer.

HRMS (ES+): Exact mass calculated for $\mathrm{C}_{17} \mathrm{H}_{16} \mathrm{NOS}^{35} \mathrm{ClF}\left[(\mathrm{M}+\mathrm{H})^{+}-\mathrm{N}_{2}\right], 336.0625$. Found $336.0630 ; \mathrm{m} / \mathrm{z} \quad(\mathrm{ES}+) \quad 338.2 \quad\left\{\left[\left(\mathrm{C}_{17} \mathrm{H}_{15} \mathrm{NOS}^{37} \mathrm{ClF}\right)+\mathrm{H}^{+}\right], \quad 44 \%\right\}, \quad 336.2$ $\left\{\left[\left(\mathrm{C}_{17} \mathrm{H}_{15} \mathrm{NOS}{ }^{35} \mathrm{ClF}\right)+\mathrm{H}^{+}\right], 100 \%\right\}, 220.2(16 \%), 88.0(48 \%)$.

\section{1,5-Dimethyl- $N$-phenyl-1 $H$-pyrazol-3-carboxamide $52^{5}$}

5-Methyl- $N$-phenyl-1 $H$-pyrazole-3-carboxamide $43(0.12 \mathrm{~g}, 0.6 \mathrm{mmol})$ in acetonitrile (15 $\mathrm{mL})$ was added to a stirring solution of potassium carbonate $(0.12 \mathrm{~g}, 0.8 \mathrm{mmol})$ in acetonitrile $(5 \mathrm{~mL})$. Methyl iodide $(0.05 \mathrm{~mL}, 0.8 \mathrm{mmol})$ was added slowly via a syringe. Following stirring under nitrogen at room temperature for $16 \mathrm{~h}$, the reaction mixture was filtered through a sintered glass funnel (grade 3) to remove the excess potassium carbonate. The solid was washed with dichloromethane $(10 \mathrm{~mL})$ and the solvent was then removed from the filtrate by evaporation under reduced pressure to give $\mathbf{5 2}$ as an orange oil. Following purification by column chromatography using hexane: ethyl acetate (gradient elution $20-80 \%$ ethyl acetate) as eluent, the pyrazole $\mathbf{5 2}$ was obtained as a white solid (0.07 g, 62\%), mp 135-137 ${ }^{\circ} \mathrm{C}$ (Lit., ${ }^{5} 142-144{ }^{\circ} \mathrm{C}$ ); (Found C, 65.52; H, 5.91; N, 18.84. $\mathrm{C}_{12} \mathrm{H}_{13} \mathrm{~N}_{3} \mathrm{O}$ requires $\left.\mathrm{C}, 66.96 ; \mathrm{H}, 6.09 ; \mathrm{N}, 19.52 \%\right) ; v_{\max } / \mathrm{cm}^{-1}(\mathrm{KBr}) 3281(\mathrm{NH})$, $1660(\mathrm{CO}), 1596,1538,1495,1429,1319 ; \delta_{\mathrm{H}}\left(300 \mathrm{MHz}, \mathrm{CDCl}_{3}\right) 2.32\left[3 \mathrm{H}, \mathrm{s}, \mathrm{C}(5) \mathrm{CH}_{3}\right]$, $3.83\left(3 \mathrm{H}, \mathrm{s}, \mathrm{NCH}_{3}\right), 6.64[1 \mathrm{H}, \mathrm{s}, \mathrm{C}(4) H], 7.05-7.16(1 \mathrm{H}, \mathrm{m}, \mathrm{ArH}), 7.29-7.41(2 \mathrm{H}, \mathrm{m}$, $\operatorname{Ar} H), 7.63-7.74(2 \mathrm{H}, \mathrm{m}, \mathrm{Ar} H), 8.65\left(1 \mathrm{H}, \mathrm{br} \mathrm{s}, \mathrm{NH}\right.$ of carboxamide); $\delta_{\mathrm{C}}(75.5 \mathrm{MHz}$, $\left.\mathrm{CDCl}_{3}\right) 11.7\left[\mathrm{CH}_{3}, \mathrm{C}(5) \mathrm{CH}_{3}\right], 37.0\left(\mathrm{CH}_{3}, \mathrm{NCH}_{3}\right), 106.8[\mathrm{CH}, \mathrm{C}(4) \mathrm{H}], 119.9,124.2,129.4$ $(3 \times \mathrm{CH}, 3 \times$ aromatic $C \mathrm{H}), 138.5(\mathrm{C}$, aromatic $C), 141.1,145.6[2 \times \mathrm{C}, C(3) \& C(5)]$, $160.5(\mathrm{C}, \mathrm{CO})$; HRMS (ES+): Exact mass calculated for $\mathrm{C}_{12} \mathrm{H}_{14} \mathrm{~N}_{3} \mathrm{O}[\mathrm{M}+\mathrm{H}]^{+}, 216.1137$. Found 216.1137; m/z (ES+) $216.2\left\{\left[\left(\mathrm{C}_{12} \mathrm{H}_{13} \mathrm{~N}_{3} \mathrm{O}\right)+\mathrm{H}^{+}\right], 100 \%\right\}$. 
$\left(3 R^{*}, 4 R^{*}, 5 R^{*}\right)-3-($ Benzylthio)-4-chloro-4,5-dihydro-5-methyl- $N$-(4-methylphenyl)$3 H$-pyrazole-3-carboxamide 45 a a n d $\left(3 R^{*}, 4 R *, 5 S^{*}\right)$-3-(benzylthio)-4-chloro-4,5dihydro-5-methyl- $N$-(4-methylphenyl)-3H-pyrazole-3-carboxamide $45 b$

An excess of an ethereal solution of diazoethane [prepared from $N$-ethyl- $N$-nitrosourea 91 $(0.71 \mathrm{~g}, 6.1 \mathrm{mmol})]$ was added to a solution of $\mathrm{N}$-(4-methylphenyl)-Z-3-chloro-2(benzylthio)propenamide $20(0.28 \mathrm{~g}, 0.9 \mathrm{mmol})$ in ether $(20 \mathrm{~mL})$ cooled in an ice-salt bath while stirring. The solution was allowed to return slowly to room temperature while stirring for $6 \mathrm{~h}$. A precipitate formed as the reaction progressed. The products were collected by filtration through a sintered glass funnel (grade 3 ) to give $\mathbf{4 5 a}$ and $\mathbf{4 5 b}$ (45a:45b 1:0.04 by ${ }^{1} \mathrm{H}$ NMR spectroscopy) as a white solid (0.22 g, 68\%), mp 114-115 ${ }^{\circ} \mathrm{C} ; v_{\max } / \mathrm{cm}^{-1}(\mathrm{KBr}) 3350(\mathrm{NH}), 3017(\mathrm{CH}), 1676(\mathrm{CO}), 1594,1520(\mathrm{~N}=\mathrm{N}$ stretch);

Major diastereomer 45a: $\delta_{\mathrm{H}}\left(300 \mathrm{MHz}, \mathrm{CDCl}_{3}\right) 1.65\left[3 \mathrm{H}, \mathrm{d}, J 7.3, \mathrm{C}(5) \mathrm{CH}_{3}\right], 2.34(3 \mathrm{H}, \mathrm{s}$, $\left.\mathrm{ArCH}_{3}\right), 4.08\left(2 \mathrm{H}, \mathrm{s}, \mathrm{SCH} H_{2}\right), 4.24[1 \mathrm{H}, \mathrm{d}, J 7.2, \mathrm{C}(4) H], 4.64-4.78[1 \mathrm{H}, \mathrm{m}, \mathrm{C}(5) H], 7.15-$ $7.61(9 \mathrm{H}, \mathrm{m}, \mathrm{Ar} H)^{*}, 8.63(1 \mathrm{H}, \mathrm{br} \mathrm{s}, \mathrm{N} H)$.

$\delta_{\mathrm{C}}\left(75.5 \mathrm{MHz}, \mathrm{CDCl}_{3}\right)$ (signals for major diastereomer $45 \mathrm{a}$ only detected) $16.0\left[\mathrm{CH}_{3}\right.$, $\left.\mathrm{C}(5) \mathrm{CH}_{3}\right], 21.3\left(\mathrm{CH}_{3}, \mathrm{ArCH} \mathrm{H}_{3}\right), 35.5\left(\mathrm{CH}_{2}, \mathrm{SCH}_{2}\right), 61.7[\mathrm{CH}, \mathrm{C}(5) \mathrm{H}], 91.7[\mathrm{CH}, \mathrm{C}(4) \mathrm{H}]$, $98.8[\mathrm{C}, C(3)], 120.3,128.2,129.2,129.6,130.0(5 \times \mathrm{CH}, 5 \times$ aromatic $\mathrm{CH}), 134.5$, 135.5, $135.8(3 \times \mathrm{C}, 3 \times$ aromatic $C), 164.6(\mathrm{C}, \mathrm{CO})$;

Minor diastereomer 45b: $\delta_{\mathrm{H}}\left(300 \mathrm{MHz}, \mathrm{CDCl}_{3}\right) 1.76\left[3 \mathrm{H}, \mathrm{d}, J 7.4, \mathrm{C}(5) \mathrm{CH}_{3}\right], 2.34(3 \mathrm{H}, \mathrm{s}$, $\left.\mathrm{ArCH}_{3}\right), 4.15(2 \mathrm{H}, \mathrm{s}, \mathrm{SCH}), 4.64-4.78[1 \mathrm{H}, \mathrm{m}, \mathrm{C}(5) H]^{*}, 5.00[1 \mathrm{H}, \mathrm{d}, J 6.4, \mathrm{C}(4) H], 7.15-$ $7.61(9 \mathrm{H}, \mathrm{m}, \mathrm{Ar} H)^{*}, 8.33(1 \mathrm{H}, \mathrm{br} \mathrm{s}, \mathrm{NH})$.

*These signals were indistinguishable for the two diastereomers.

HRMS (ES+): Exact mass calculated for $\mathrm{C}_{19} \mathrm{H}_{21} \mathrm{NOS}^{35} \mathrm{Cl}\left[(\mathrm{M}+\mathrm{H})^{+}-\mathrm{N}_{2}\right], 346.1032$. Found $346.1031 ; \mathrm{m} / \mathrm{z}(\mathrm{ES}+) 348.0\left\{\left[\left(\mathrm{C}_{19} \mathrm{H}_{20} \mathrm{NOS}^{37} \mathrm{Cl}\right)+\mathrm{H}^{+}\right], \quad 36 \%\right\}, \quad 346.0$ $\left\{\left[\left(\mathrm{C}_{19} \mathrm{H}_{20} \mathrm{NOS}^{35} \mathrm{Cl}\right)+\mathrm{H}^{+}\right], 88 \%\right\}, 244.1(100 \%)$.

Elemental analysis resulted in an underestimation of the carbon, hydrogen and nitrogen content, possibly indicating decomposition or the presence of an inorganic impurity. 
$\left(3 R^{*}, 4 R^{*}, 5 R^{*}\right)-3-($ Benzylthio)- $N$-n-butyl-4-chloro-4,5-dihydro-5-methyl-3Hpyrazole-3-carboxamide $46 \mathrm{a}$ and $\left(3 R^{*}, 4 R^{*}, 5 S^{*}\right)-3$-(benzylthio)- $N$-n-butyl-4-chloro4,5-dihydro-5-methyl-3H-pyrazole-3-carboxamide 46b

An excess of an ethereal solution of diazoethane [prepared from $N$-ethyl- $N$-nitrosourea 91 $(0.97 \mathrm{~g}, 8.3 \mathrm{mmol})]$ was added to a solution of $N$ - $n$-butyl-Z-3-chloro-2(benzylthio)propenamide $21(0.34 \mathrm{~g}, 1.2 \mathrm{mmol})$ in ether $(25 \mathrm{~mL})$ cooled in an ice-salt bath while stirring. The reaction solution was allowed to return slowly to room temperature while stirring for $6 \mathrm{~h}$. The solvent was removed by evaporation at reduced pressure to give the crude products 46a and 46b (46a:46b 1:0.16 by ${ }^{1} \mathrm{H}$ NMR spectroscopy) as a colourless oil. Crystallisation from ether/hexane gave 46a and 46b (46a:46b 1:0.10 by ${ }^{1} \mathrm{H}$ NMR spectroscopy) as a white solid (0.17g, 42\%), mp 61-62 ${ }^{\circ} \mathrm{C}$; $v_{\max } / \mathrm{cm}^{-1}(\mathrm{KBr}) 3355(\mathrm{NH}), 3017(\mathrm{CH}), 2953(\mathrm{CH}), 1664(\mathrm{CO}), 1521(\mathrm{~N}=\mathrm{N}$ stretch); Major diastereomer 46a: $\delta_{\mathrm{H}}\left(300 \mathrm{MHz}, \mathrm{CDCl}_{3}\right)$ 0.86-0.99 [3H, m, $\left.J 7.4, \mathrm{C}\left(4^{\prime}\right) H_{3}\right]^{*}, 1.23-$ $1.68\left[7 \mathrm{H}, \mathrm{m}, \mathrm{C}\left(3^{\prime}\right) \mathrm{H}_{2}, \mathrm{C}\left(2^{\prime}\right) \mathrm{H}_{2} \& \mathrm{C}(5) \mathrm{CH}_{3}\right]^{*}, 3.16-3.38\left(2 \mathrm{H}, \mathrm{m}, \mathrm{NHCH}_{2}\right)^{*}, 3.99(1 \mathrm{H}, \mathrm{d}, \mathrm{A}$ of $\mathrm{AB}$ system, $\left.J_{\mathrm{AB}} 11.5, \mathrm{SCH}_{2}\right), 4.03\left(1 \mathrm{H}, \mathrm{d}, \mathrm{B} \text { of } \mathrm{AB} \text { system, } J_{\mathrm{AB}} 11.7, \mathrm{SCH}\right)_{2}, 4.17[1 \mathrm{H}$, d, J 7.0, C(4)H], 4.56-4.72 [1H, m, C $(5) H]^{*}, 6.89(1 \mathrm{H}$, br s, NH), 7.22-7.44 (5H, m, $\operatorname{Ar} H)^{*} ; \delta_{\mathrm{C}}\left(75.5 \mathrm{MHz}, \mathrm{CDCl}_{3}\right) 12.0,13.9\left[2 \times \mathrm{CH}_{3}, C\left(4^{\prime}\right) \mathrm{H}_{3} \& C(5) \mathrm{H}_{3}\right], 18.4\left[\mathrm{CH}_{2}\right.$, $\left.C\left(3^{\prime}\right) \mathrm{H}_{2}\right], 29.7\left[\mathrm{CH}_{2}, C\left(2^{\prime}\right) \mathrm{H}_{2}\right], 33.3^{\S}, 38.2\left(2 \times \mathrm{CH}_{2}, \mathrm{SCH}_{2} \& \mathrm{NHCH}_{2}\right), 59.8[\mathrm{CH}$, $C(5) \mathrm{H}]^{\S}, 89.5[\mathrm{CH}, C(4) \mathrm{H}]^{\S}, 96.5[C(3)]^{\S}, 164.6(\mathrm{C}, C \mathrm{O})^{\S}$.

Minor diastereomer 46b: $\delta_{\mathrm{H}}\left(300 \mathrm{MHz}, \mathrm{CDCl}_{3}\right)$ 0.86-0.99 [3H, m, $J$ 7.4, C(4') $\left.H_{3}\right]^{*}, 1.23-$ $1.68\left[4 \mathrm{H}, \mathrm{m}, \mathrm{C}\left(3^{\prime}\right) H_{2} \& \mathrm{C}\left(2^{\prime}\right) H_{2}\right]^{*}, 1.75\left[3 \mathrm{H}, \mathrm{d}, J 7.3, \mathrm{C}(5) \mathrm{CH}_{3}\right], 3.16-3.38(2 \mathrm{H}, \mathrm{m}$, $\left.\mathrm{NHCH}_{2}\right)^{*}, 4.10\left(2 \mathrm{H}, \mathrm{d}, J 3.4, \mathrm{SCH}_{2}\right), 4.56-4.72[1 \mathrm{H}, \mathrm{m}, \mathrm{C}(5) H]^{*}, 4.92[1 \mathrm{H}, \mathrm{d}, J 6.0$, $\mathrm{C}(4) H], 6.59(1 \mathrm{H}$, br s, $\mathrm{NH}), 7.22-7.44(5 \mathrm{H}, \mathrm{m}, \mathrm{Ar} H)^{*} ; \delta_{\mathrm{C}}\left(75.5 \mathrm{MHz}, \mathrm{CDCl}_{3}\right) 12.1\left[\mathrm{CH}_{3}\right.$, $C\left(4^{\prime}\right) \mathrm{H}_{3}$ or $\left.C(5) \mathrm{H}_{3}\right], 18.5\left[\mathrm{CH}_{2}, C\left(3^{\prime}\right) \mathrm{H}_{2}\right], 29.5\left[\mathrm{CH}_{2}, C\left(2^{\prime}\right) \mathrm{H}_{2}\right], 37.8\left(\mathrm{CH}_{2}, \mathrm{SCH}_{2}\right)$.

*These signals were indistinguishable for the two diastereomers in the ${ }^{1} \mathrm{H}$ NMR spectrum.

${ }^{\S}$ The analogous signals were not detected for the minor diastereomer.

The aromatic signals were not distinguished in the ${ }^{13} \mathrm{C}$ NMR for the two diastereomers and were seen at $\delta_{\mathrm{C}} 126.1,126.4,127.1,127.2,127.6(5 \times \mathrm{CH}, 5 \times$ aromatic $\mathrm{CH}), 134.0$ $(\mathrm{C}$, aromatic $C)$. 
HRMS (ES+): Exact mass calculated for $\mathrm{C}_{16} \mathrm{H}_{23} \mathrm{NOS}^{35} \mathrm{Cl}\left[(\mathrm{M}+\mathrm{H})^{+}-\mathrm{N}_{2}\right], 312.1189$. Found $312.1193 ; \mathrm{m} / \mathrm{z}(\mathrm{ES}+) 314.2\left\{\left[\left(\mathrm{C}_{16} \mathrm{H}_{22} \mathrm{NOS}^{37} \mathrm{Cl}\right)+\mathrm{H}^{+}\right], \quad 42 \%\right\}, \quad 312.2$ $\left\{\left[\left(\mathrm{C}_{16} \mathrm{H}_{22} \mathrm{NOS}^{35} \mathrm{Cl}\right)+\mathrm{H}^{+}\right], 100 \%\right\}, 214.1(10 \%)$.

$\left(3 R^{*}, 4 R^{*}, 5 R^{*}\right)-N$-Benzyl-3-(phenylthio)-4-chloro-4,5-dihydro-5-methyl-3Hpyrazole-3-carboxamide $48 \mathrm{a}$ and $\left(3 R^{*}, 4 R^{*}, 5 S^{*}\right)-N$-benzyl-3-(phenylthio)-4-chloro4,5-dihydro-5-methyl-3 $\mathrm{H}$-pyrazole-3-carboxamide $48 \mathrm{~b}$

An excess of an ethereal solution of diazoethane [prepared from $N$-ethyl- $N$-nitrosourea 91 $(0.87 \mathrm{~g}, 7.5 \mathrm{mmol})]$ was added to a solution of $N$-benzyl-Z-3-chloro-2(phenylthio)propenamide $23(0.32 \mathrm{~g}, 1.1 \mathrm{mmol})$ in ether $(25 \mathrm{~mL})$ cooled in an ice-salt bath while stirring. The reaction solution was allowed to return slowly to room temperature while stirring for $6 \mathrm{~h}$. The solvent was removed by evaporation at reduced pressure to give the crude products $48 \mathrm{a}$ and $48 \mathrm{~b}$ (48a:48b 1:0.27 by ${ }^{1} \mathrm{H}$ NMR spectroscopy) as a colourless oil. Crystallisation from ether/hexane gave $48 \mathrm{a}$ and $\mathbf{4 8 b}$ (48a:48b 1:0.22 by ${ }^{1} \mathrm{H}$ NMR spectroscopy) as a white solid (0.31 g, 80\%), mp 73-74 ${ }^{\circ} \mathrm{C}$; (Found $\mathrm{C}, 59.51 ; \mathrm{H}, 4.94 ; \mathrm{N}, 11.82 . \mathrm{C}_{18} \mathrm{H}_{18} \mathrm{ClN}_{3} \mathrm{OS}$ requires $\mathrm{C}, 60.07 ; \mathrm{H}, 5.04 ; \mathrm{N}$, 11.68\%); $v_{\max } / \mathrm{cm}^{-1}(\mathrm{KBr}) 3357(\mathrm{NH}), 3058(\mathrm{CH}), 2978(\mathrm{CH}), 1673(\mathrm{CO}), 1518(\mathrm{~N}=\mathrm{N}$ stretch);

Major diastereomer 48a: $\delta_{\mathrm{H}}\left(300 \mathrm{MHz}, \mathrm{CDCl}_{3}\right) 1.50\left[3 \mathrm{H}, \mathrm{d}, J 7.4, \mathrm{C}(5) \mathrm{CH}_{3}\right], 4.08(1 \mathrm{H}$, dd, A of ABX, $J_{\mathrm{AB}} 14.6, J_{\mathrm{AX}} 5.4$, one of $\left.\mathrm{NHCH}_{2}\right), 4.24[1 \mathrm{H}, \mathrm{d}, J 6.5, \mathrm{C}(4) H], 4.30(1 \mathrm{H}$, dd, B of ABX, $J_{\mathrm{AB}} 14.6, J_{\mathrm{BX}} 5.9$, one of $\left.\mathrm{NHCH}_{2}\right), 4.48-4.57$ [1H, sym m, C(5)H], 6.38 $(1 \mathrm{H}$, br s, $\mathrm{NH}), 7.18-7.46(8 \mathrm{H}, \mathrm{m}, \mathrm{ArH})^{*}, 7.59-7.68(2 \mathrm{H}, \mathrm{m}, \mathrm{Ar} H) ; \delta_{\mathrm{C}}\left(75.5 \mathrm{MHz}, \mathrm{CDCl}_{3}\right)$ $15.9\left[\mathrm{C}(5) \mathrm{CH}_{3}\right], 44.4\left(\mathrm{NHCH}_{2}\right), 61.0[C(5) \mathrm{H}], 91.3[C(4) \mathrm{H}], 99.1[C(3)]^{\S}, 166.4(C \mathrm{O})$.

Minor diastereomer 48b: $\delta_{\mathrm{H}}\left(300 \mathrm{MHz}, \mathrm{CDCl}_{3}\right) 1.77\left[3 \mathrm{H}, \mathrm{d}, J 7.2, \mathrm{C}(5) \mathrm{CH}_{3}\right], 3.77(1 \mathrm{H}$, dd, A of $\mathrm{ABX}, J_{\mathrm{AB}} 14.7, J_{\mathrm{AX}} 5.1$, one of $\left.\mathrm{NHCH}_{2}\right), 4.14\left(1 \mathrm{H}\right.$, dd, B of $\mathrm{ABX}, J_{\mathrm{AB}} 14.7, J_{\mathrm{BX}}$ 6.4, one of $\left.\mathrm{NCH}_{2}\right), 4.32-4.40$ [1H, sym m, C $\left.(5) H\right], 5.01[1 \mathrm{H}, \mathrm{d}, J 5.8, \mathrm{C}(4) H], 5.65(1 \mathrm{H}$, br s, NH), 6.79-6.87 (2H, m, $\operatorname{Ar} H), 7.18-7.46(6 \mathrm{H}, \mathrm{m}, \operatorname{Ar} H)^{*}, 7.72-7.79(2 \mathrm{H}, \mathrm{m}, \operatorname{Ar} H) ; \delta_{\mathrm{C}}$ $\left(75.5 \mathrm{MHz}, \mathrm{CDCl}_{3}\right) 14.1\left[\mathrm{C}(5) \mathrm{CH}_{3}\right], 44.6\left(\mathrm{NHCH}_{2}\right), 62.0[C(5) \mathrm{H}], 85.8[C(4) \mathrm{H}], 165.1$ $(\mathrm{CO})$.

*The aromatic signals were indistinguishable for the two diastereomers in the ${ }^{1} \mathrm{H}$ NMR spectrum. 
${ }^{\S}$ The analogous signal was not detected for the minor diastereomer.

The aromatic signals were not distinguished in the ${ }^{13} \mathrm{C}$ NMR spectrum for the two diastereomers and were seen at $\delta_{\mathrm{C}} 128.18,128.24,128.4,128.7,129.1,129.5,129.6$, 130.4, 130.6, 136.9, 137.6 (aromatic $C H$ \& aromatic $C$ ).

HRMS (ES+): Exact mass calculated for $\mathrm{C}_{18} \mathrm{H}_{19} \mathrm{NOS}^{35} \mathrm{Cl}\left[(\mathrm{M}+\mathrm{H})^{+}-\mathrm{N}_{2}\right], 332.0876$. Found $332.0887 ; \mathrm{m} / \mathrm{z}(\mathrm{ES}+) 334.2\left\{\left[\left(\mathrm{C}_{18} \mathrm{H}_{18} \mathrm{NOS}^{37} \mathrm{Cl}\right)+\mathrm{H}^{+}\right], \quad 24 \%\right\}, \quad 332.2$ $\left\{\left[\left(\mathrm{C}_{18} \mathrm{H}_{18} \mathrm{NOS}^{35} \mathrm{Cl}\right)+\mathrm{H}^{+}\right], 40 \%\right\}, 88.0(26 \%)$.

\section{$(3 R *, 4 R *, 5 R *)-3-($ Phenylthio)-4-chloro-4,5-dihydro-5-methyl- $N$-(4-methylphenyl)-}

\section{$3 H$-pyrazole-3-carboxamide 49 a a n d $\left(3 R^{*}, 4 R^{*}, 5 S^{*}\right)-3$-(phenylthio)-4-chloro-4,5-} dihydro-5-methyl- $\mathrm{N}$-(4-methylphenyl)-3H-pyrazole-3-carboxamide 49b

An excess of an ethereal solution of diazoethane [prepared from $N$-ethyl- $N$-nitrosourea 91 $(0.76 \mathrm{~g}, 6.5 \mathrm{mmol})]$ was added to a solution of $N$-(4-methylphenyl)-Z-3-chloro-2(phenylthio)propenamide $24(0.28 \mathrm{~g}, 0.9 \mathrm{mmol})$ in ether $(25 \mathrm{~mL})$ cooled in an ice-salt bath while stirring. The reaction solution was allowed to return slowly to room temperature while stirring for $6 \mathrm{~h}$. The solvent was removed by evaporation at reduced pressure to give the crude products 49a and 49b (49a:49b 1:0.27 by ${ }^{1} \mathrm{H}$ NMR spectroscopy) as a pale yellow oil. Purification by column chromatography on neutral alumina (activity II) using hexane-ethyl acetate 98:2 gave 49a as a low melting white solid $(0.16 \mathrm{~g}, 46 \%)$ and a single diastereomer; $v_{\max } / \mathrm{cm}^{-1}(\mathrm{KBr}) 3376(\mathrm{NH}), 2979(\mathrm{CH})$, $1689(\mathrm{CO}), 1522(\mathrm{~N}=\mathrm{N}$ stretch);

Major diastereomer 49a: $\delta_{\mathrm{H}}\left(300 \mathrm{MHz}, \mathrm{CDCl}_{3}\right) 1.55\left[3 \mathrm{H}, \mathrm{d}, J 7.4, \mathrm{C}(5) \mathrm{CH}_{3}\right], 2.29(3 \mathrm{H}, \mathrm{s}$, $\left.\mathrm{ArCH}_{3}\right), 4.30[1 \mathrm{H}, \mathrm{d}, J 6.6, \mathrm{C}(4) H], 4.57-4.67[1 \mathrm{H}, \mathrm{m}, \mathrm{C}(5) H], 7.01-7.11(4 \mathrm{H}, \mathrm{m}, \mathrm{ArH} \&$ $\mathrm{NH}), 7.27-7.44(3 \mathrm{H}, \mathrm{m}, \mathrm{ArH}), 7.63-7.74(3 \mathrm{H}, \mathrm{m}, \mathrm{ArH}) ; \delta_{\mathrm{C}}\left(75.5 \mathrm{MHz}, \mathrm{CDCl}_{3}\right) 16.1\left[\mathrm{CH}_{3}\right.$, $\left.\mathrm{C}(5) \mathrm{CH}_{3}\right], 21.5\left(\mathrm{CH}_{3}, \mathrm{ArCH}_{3}\right), 61.0[\mathrm{CH}, \mathrm{C}(5) \mathrm{H}], 91.8[\mathrm{CH}, \mathrm{C}(4) \mathrm{H}], 99.0[\mathrm{C}, \mathrm{C}(3)]$, $120.5(\mathrm{CH}$, aromatic $\mathrm{CH}), 128.5(\mathrm{C}$, aromatic $C), 129.8,130.0,131.0(3 \times \mathrm{CH}, 3 \times$ aromatic $C H), 134.2,135.4(2 \times \mathrm{C}, 2 \times$ aromatic $C), 138.1(\mathrm{CH}$, aromatic $C \mathrm{H}), 164.5(\mathrm{C}$, $\mathrm{CO})$.

The minor diastereomer 49b was seen in the ${ }^{1} \mathrm{H}$ NMR spectrum of the crude product (ratio of major to minor 1:0.27 by ${ }^{1} \mathrm{H}$ NMR spectroscopy): $\delta_{\mathrm{H}}\left(300 \mathrm{MHz}, \mathrm{CDCl}_{3}\right) 1.79$ 
$\left[3 \mathrm{H}, \mathrm{d}, J 7.2, \mathrm{C}(5) \mathrm{CH}_{3}\right], 2.29\left(3 \mathrm{H}, \mathrm{s}, \mathrm{ArCH}_{3}\right), 4.44-4.55[1 \mathrm{H}, \mathrm{m}, \mathrm{C}(5) H], 5.08[1 \mathrm{H}, \mathrm{d}, J$ 5.9, $\mathrm{C}(4) H], 6.89-7.86(10 \mathrm{H}, \mathrm{m}, \mathrm{Ar} H \& \mathrm{~N} H)$.

HRMS (ES+): Exact mass calculated for $\mathrm{C}_{18} \mathrm{H}_{19} \mathrm{NOS}^{35} \mathrm{Cl}\left[(\mathrm{M}+\mathrm{H})^{+}-\mathrm{N}_{2}\right], 332.0876$. Found $332.0891 ; \mathrm{m} / \mathrm{z}(\mathrm{ES}+) \quad 334.2 \quad\left\{\left[\left(\mathrm{C}_{18} \mathrm{H}_{18} \mathrm{NOS}^{37} \mathrm{Cl}\right)+\mathrm{H}^{+}\right], \quad 42 \%\right\}, \quad 332.2$ $\left\{\left[\left(\mathrm{C}_{18} \mathrm{H}_{18} \mathrm{NOS}^{35} \mathrm{Cl}\right)+\mathrm{H}^{+}\right], 100 \%\right\}$.

$\left(3 R^{*}, 4 R^{*}, 5 R^{*}\right)-3-($ Phenylthio)-4-chloro-4,5-dihydro- $N, 5$-dimethyl-3H-pyrazole-3carboxamide 50 a a n d $\left(3 R^{*}, 4 R^{*}, 5 S^{*}\right)-3$-(phenylthio)-4-chloro-4,5-dihydro- $N, 5$ dimethyl-3H-pyrazole-3-carboxamide 50b

An excess of an ethereal solution of diazoethane [prepared from $N$-ethyl- $N$-nitrosourea 91 $(1.20 \mathrm{~g}, 10.3 \mathrm{mmol})]$ was added to a solution of $N$-methyl-Z-3-chloro-2(phenylthio)propenamide $25(0.33 \mathrm{~g}, 1.5 \mathrm{mmol})$ in ether $(25 \mathrm{~mL})$ cooled in an ice-salt bath while stirring. The reaction solution was allowed to return slowly to room temperature while stirring for $6 \mathrm{~h}$. The solvent was removed by evaporation at reduced pressure to give the crude products 50a and 50b (50a:50b 1:0.30 by ${ }^{1} \mathrm{H}$ NMR spectroscopy) as a white solid. Crystallisation from ether/hexane gave 50a and 50b (50a:50b 1:0.10 by ${ }^{1} \mathrm{H}$ NMR spectroscopy) as a white solid $(0.20 \mathrm{~g}, 47 \%)$, mp $75-76{ }^{\circ} \mathrm{C}$; (Found C, 50.94; H, 4.92; N, 15.32; Cl, 10.21; S, 11.94. $\mathrm{C}_{12} \mathrm{H}_{14} \mathrm{ClN}_{3} \mathrm{OS}$ requires $\mathrm{C}$, 50.79; H, 4.97; N, 14.81; Cl, 12.49; S, 11.30\%); $v_{\max } / \mathrm{cm}^{-1}(\mathrm{KBr}) 3343(\mathrm{NH}), 2951(\mathrm{CH})$, $1672(\mathrm{CO}), 1544(\mathrm{~N}=\mathrm{N}$ stretch$)$;

Major diastereomer 50a: $\delta_{\mathrm{H}}\left(300 \mathrm{MHz}, \mathrm{CDCl}_{3}\right) 1.47\left[3 \mathrm{H}, \mathrm{d}, J 7.2, \mathrm{C}(5) \mathrm{CH} H_{3}\right], 2.56(3 \mathrm{H}, \mathrm{d}$, $\left.J 4.8, \mathrm{NHCH}_{3}\right), 4.25[1 \mathrm{H}, \mathrm{d}, J 6.3, \mathrm{C}(4) H], 4.55[1 \mathrm{H}, \mathrm{dq}, J 7.2,6.3, \mathrm{C}(5) H], 6.00(1 \mathrm{H}, \mathrm{br}$ s, $\mathrm{NH}), 7.33-7.50(3 \mathrm{H}, \mathrm{m}, \operatorname{Ar} H)^{*}, 7.62-7.73(2 \mathrm{H}, \mathrm{m}, \operatorname{Ar} H)^{*} ; \delta_{\mathrm{C}}\left(75.5 \mathrm{MHz}, \mathrm{CDCl}_{3}\right) 16.0$ $\left[\mathrm{CH}_{3}, \mathrm{C}(5) \mathrm{CH}_{3}\right], 26.7\left(\mathrm{CH}_{3}, \mathrm{NHCH}_{3}\right)^{\S}, 60.7[\mathrm{CH}, \mathrm{C}(5) \mathrm{H}], 91.5[\mathrm{CH}, \mathrm{C}(4) \mathrm{H}], 99.6[\mathrm{C}$, $C(3)]^{\S}, 129.0(\mathrm{C} \text {, aromatic } C)^{\S}, 129.5,130.7,137.8(3 \times \mathrm{CH}, 3 \times$ aromatic $C H), 167.1(\mathrm{C}$, $(\mathrm{O})^{\S}$.

Minor diastereomer 50b: $\delta_{\mathrm{H}}\left(300 \mathrm{MHz}, \mathrm{CDCl}_{3}\right) 1.77\left[3 \mathrm{H}, \mathrm{d}, J 7.2, \mathrm{C}(5) \mathrm{CH}_{3}\right], 2.33(3 \mathrm{H}$, d, $J$ 5.1, $\left.\mathrm{NHCH}_{3}\right), 4.32[1 \mathrm{H}, \mathrm{dq}, J 7.2,5.7, \mathrm{C}(5) H], 5.00[1 \mathrm{H}, \mathrm{d}, J 5.7, \mathrm{C}(4) H], 5.25(1 \mathrm{H}$, br s, $\mathrm{N} H), 7.33-7.50(2 \mathrm{H}, \mathrm{m}, \operatorname{Ar} H)^{*}, 7.62-7.73(2 \mathrm{H}, \mathrm{m}, \operatorname{Ar} H)^{*}, 7.74-7.81(1 \mathrm{H}, \mathrm{m}, \operatorname{Ar} H)$; $\delta_{\mathrm{C}}\left(75.5 \mathrm{MHz}, \mathrm{CDCl}_{3}\right) 14.0\left[\mathrm{CH}_{3}, \mathrm{C}(5) \mathrm{CH}_{3}\right], 61.9[\mathrm{CH}, \mathrm{C}(5) \mathrm{H}], 85.6[\mathrm{CH}, \mathrm{C}(4) \mathrm{H}], 129.4$, $130.5,137.3(3 \times \mathrm{CH}, 3 \times$ aromatic $\mathrm{CH})$. 
*The aromatic signals were indistinguishable for the two diastereomers.

${ }^{\S}$ The analogous signals were not detected for the minor diastereomer.

HRMS (ES+): Exact mass calculated for $\mathrm{C}_{12} \mathrm{H}_{15} \mathrm{NOS}^{35} \mathrm{Cl}\left[(\mathrm{M}+\mathrm{H})^{+}-\mathrm{N}_{2}\right], 256.0563$. Found $256.0571 ; \mathrm{m} / \mathrm{z}(\mathrm{ES}+) \quad 258.1 \quad\left\{\left[\left(\mathrm{C}_{12} \mathrm{H}_{14} \mathrm{NOS}^{37} \mathrm{Cl}\right)+\mathrm{H}^{+}\right], \quad 40 \%\right\}, \quad 256.2$ $\left\{\left[\left(\mathrm{C}_{12} \mathrm{H}_{14} \mathrm{NOS}^{35} \mathrm{Cl}\right)+\mathrm{H}^{+}\right], 100 \%\right\}, 220.2(52 \%)$.

$\left(3 R *, 4 R^{*}, 5 R^{*}\right)-4-C h l o r o-4,5-d i h y d r o-N, 5-d i m e t h y l-3-($ phenylthio)-3H-pyrazole-3carboxamide 55a \& $\left(3 R^{*}, 4 R^{*}, 5 S^{*}\right)-4-c h l o r o-4,5-d i h y d r o-N, 5$-dimethyl-3(phenylthio)-3H-pyrazole-3-carboxamide 55b

An excess of an ethereal solution of diazoethane [prepared from $N$-ethyl- $N$-nitrosourea 91 $(0.80 \mathrm{~g}, 6.8 \mathrm{mmol})]$ was added to a solution of methyl Z-3-chloro-2(phenylthio)propenoate $54(0.25 \mathrm{~g}, 1.0 \mathrm{mmol})$ in ether $(25 \mathrm{~mL})$ at $-20{ }^{\circ} \mathrm{C}$. The reaction solution was allowed to return slowly to room temperature and then stirred for $6 \mathrm{~h}$. The solvent was removed by evaporation at reduced pressure to give the crude products 55a and 55b as a colourless oil and a 1:0.23 mixture of diastereomers. Purification by column chromatography on a short column of neutral alumina (activity II) using hexane-ethyl acetate $98: 2$ as eluent gave the major diastereomer 55a as a clear oil $(0.13 \mathrm{~g}, 48 \%)$; $v_{\max } / \mathrm{cm}^{-1}(\mathrm{KBr}) 2953(\mathrm{CH}), 1738(\mathrm{CO}), 1250 ; \delta_{\mathrm{H}} 1.47\left[3 \mathrm{H}, \mathrm{d}, J 7.5, \mathrm{C}(5) \mathrm{CH}_{3}\right], 3.62(3 \mathrm{H}$, s, $\left.\mathrm{OCH}_{3}\right), 4.17[1 \mathrm{H}, \mathrm{d}, J 6.3, \mathrm{C}(4) H], 4.47$ [1H, overlapping dq, $\left.J 7.5,6.3, \mathrm{C}(5) H\right], 7.32-$ $7.47(3 \mathrm{H}, \mathrm{m}, \mathrm{ArH}), 7.66-7.76(2 \mathrm{H}, \mathrm{m}, \mathrm{ArH}) ; \delta_{\mathrm{C}}\left(75.5 \mathrm{MHz}, \mathrm{CDCl}_{3}\right) 15.5\left[\mathrm{CH}_{3}, \mathrm{C}(5) \mathrm{CH}_{3}\right]$, $53.4\left(\mathrm{CH}_{3}, \mathrm{OCH}_{3}\right), 61.0[\mathrm{CH}, C(5) \mathrm{H}], 90.5[\mathrm{CH}, C(4) \mathrm{H}], 99.3[\mathrm{C}, C(3)], 128.1(\mathrm{C}$, aromatic $C), 129.0,130.3,137.2,(3 \times \mathrm{CH}$, aromatic $C \mathrm{H}), 167.4(\mathrm{C}, C \mathrm{O})$; HRMS (ES+): Exact mass calculated for $\mathrm{C}_{12} \mathrm{H}_{13} \mathrm{~N}_{2} \mathrm{O}_{2} \mathrm{~S}\left[(\mathrm{M}+\mathrm{H})^{+}-\mathrm{HCl}\right], 249.0698$. Found $249.0705 ; \mathrm{m} / \mathrm{z}$ $(\mathrm{ES}+) 287.1\left\{\left[\left(\mathrm{C}_{12} \mathrm{H}_{13} \mathrm{~N}_{2} \mathrm{O}_{2} \mathrm{~S}^{37} \mathrm{Cl}\right)+\mathrm{H}^{+}\right], 16 \%\right\}, 285.1\left\{\left[\left(\mathrm{C}_{12} \mathrm{H}_{13} \mathrm{~N}_{2} \mathrm{O}_{2} \mathrm{~S}^{35} \mathrm{Cl}\right)+\mathrm{H}^{+}\right], 28 \%\right\}$, $249.1\left\{\left[\left(\mathrm{C}_{12} \mathrm{H}_{12} \mathrm{~N}_{2} \mathrm{O}_{2} \mathrm{~S}\right)+\mathrm{H}^{+}\right], 30 \%\right\}$.

The minor diastereomer $\mathbf{5 5 b}$ was evident in the ${ }^{1} \mathrm{H}$ NMR spectrum of the crude product: $\delta_{\mathrm{H}} 1.78\left[3 \mathrm{H}, \mathrm{d}, J 7.2, \mathrm{C}(5) \mathrm{CH}_{3}\right], 3.34\left(3 \mathrm{H}, \mathrm{s}, \mathrm{OCH}_{3}\right), 4.42-4.51[1 \mathrm{H}, \mathrm{m}, \mathrm{C}(5) H], 4.82[1 \mathrm{H}$, d, $J$ 5.4, C(4)H], 7.32-7.47 (3H, m, $\operatorname{Ar} H)^{*}, 7.80-7.91(2 \mathrm{H}, \mathrm{m}, \operatorname{Ar} H)$.

*These signals were overlapping with the signals for the major diastereomer 55 a.

\section{$N$-Benzyl-1 H-pyrazole-3-carboxamide 58}



trimethylsilyldiazomethane

An ethereal solution of trimethylsilyldiazomethane (1.53 mL of a $2 \mathrm{M}$ solution, $3.1 \mathrm{mmol})$ was added to a stirring solution of $N$-benzyl-Z-3-chloro-2-(benzylsulfinyl)propenamide $10(0.20 \mathrm{~g}, 0.6 \mathrm{mmol})$ in ether $(25 \mathrm{~mL})$ and acetone $(4 \mathrm{~mL})$ under nitrogen at room temperature in the dark. The reaction solution was then stirred for $6 \mathrm{~h}$. The solvent and excess trimethylsilyldiazomethane were removed by evaporation at reduced pressure to give the crude product as a yellow solid. Purification by column chromatography using hexane-ethyl acetate (gradient elution $20-40 \%$ ethyl acetate) gave $\mathbf{5 8}$ as a white solid (0.08 g, 66\%), mp 149-150 ${ }^{\circ} \mathrm{C}$; (Found $\mathrm{C}, 65.20 ; \mathrm{H}, 5.43 ; \mathrm{N}, 19.97 . \mathrm{C}_{11} \mathrm{H}_{11} \mathrm{~N}_{3} \mathrm{O}$ requires C, 65.66; H, 5.51; N, 20.88\%); $v_{\max } / \mathrm{cm}^{-1}$ (KBr) 3292 (NH stretch), 1641 (CO), 1555, $1351 ; \delta_{\mathrm{H}}\left(300 \mathrm{MHz}, \mathrm{DMSO}-d_{6}\right) 4.45\left(2 \mathrm{H}, \mathrm{d}, J 6.3, \mathrm{NHCH}_{2}\right), 6.68[1 \mathrm{H}$, br s, C(4)H], 7.18$7.36(5 \mathrm{H}, \mathrm{m}, \mathrm{Ar} H), 7.82$ [1H, br s, C(5)H], $8.68(1 \mathrm{H}$, br s, $\mathrm{NH}$ of carboxamide), 13.24 $[1 \mathrm{H}$, br s, $\mathrm{N}(1) H] ; \delta_{\mathrm{C}}\left(75.5 \mathrm{MHz}, \mathrm{DMSO}-d_{6}\right) 42.2\left(\mathrm{CH}_{2}, \mathrm{NHCH}_{2}\right), 105.4[\mathrm{CH}, C(4) \mathrm{H}]$, 127.0, 127.6, 128.6, 130.3 [4 $\times \mathrm{CH}$, aromatic $C \mathrm{H} \& C(5) \mathrm{H}], 140.3(\mathrm{C}$, aromatic $C), 146.9$ [C, $C(3)], 162.2(\mathrm{C}, C \mathrm{O})$; HRMS (ES+): Exact mass calculated for $\mathrm{C}_{11} \mathrm{H}_{12} \mathrm{~N}_{3} \mathrm{O}[\mathrm{M}+\mathrm{H}]^{+}$, 202.0980. Found 202.0980; m/z (ES+) $202.2\left\{\left[\left(\mathrm{C}_{11} \mathrm{H}_{11} \mathrm{~N}_{3} \mathrm{O}\right)+\mathrm{H}^{+}\right], 100 \%\right\}, 90.9(18 \%)$.

b) Prepared from N-benzyl-Z-3-chloro-2-(benzenesulfinyl)propenamide $\mathbf{1 4}$ and trimethylsilyldiazomethane

The title compound was also prepared using an ethereal solution of trimethylsilyldiazomethane $(2.21 \mathrm{~mL}$ of a $2 \mathrm{M}$ solution, $4.4 \mathrm{mmol})$ and $\mathrm{N}$-benzyl-Z-3chloro-2-(benzenesulfinyl)propenamide $14(0.28 \mathrm{~g}, 0.9 \mathrm{mmol})$ in ether $(25 \mathrm{~mL})$ and acetone $(4 \mathrm{~mL})$. Following stirring at room temperature for $6 \mathrm{~h}$ and removal of the solvent and excess trimethylsilyldiazomethane, the crude product was obtained as a yellow solid. Following purification by column chromatography using hexane-ethyl acetate (gradient elution 20-40\% ethyl acetate), 58 was obtained as a white solid (0.04 g, $25 \%$ ), with IR and ${ }^{1} \mathrm{H}$ NMR spectroscopic details identical to above, except signals at $\delta_{\mathrm{H}}$ 6.68 and 7.82 are notably sharper and appear as two doublets $(J, 2.1)$ and NH signal is split into a broad triplet $(J, 5.7)$.

c) Prepared from N-benzyl-Z-3-chloro-2-(benzenesulfinyl)propenamide $\mathbf{1 4}$ and diazomethane 
The title compound was also prepared by adding a solution of $N$-benzyl-Z-3-chloro-2(benzenesulfinyl)propenamide $14(0.16 \mathrm{~g}, 0.5 \mathrm{mmol})$ in ether $(15 \mathrm{~mL})$ and acetone $(4$ $\mathrm{mL})$ to an ethereal solution of diazomethane [prepared from Diazald $\left.{ }^{\circledR}(0.84 \mathrm{~g}, 3.9 \mathrm{mmol})\right]$ cooled in an ice-salt bath while stirring. The reaction solution was slowly allowed to return to room temperature, and following stirring for $6 \mathrm{~h}$ and removal of the solvent and excess diazomethane, the crude product was obtained as a yellow oil. Following purification by column chromatography using hexane-ethyl acetate (gradient elution 20$40 \%$ ethyl acetate), 39 was obtained as a white solid (0.04 g, 40\%), with IR and ${ }^{1} \mathrm{H}$ NMR spectroscopic details identical to above, except the signal at $\delta_{\mathrm{H}} 6.68 \mathrm{ppm}$ was notably sharper.

\section{$\mathrm{N}$-(4-Fluorophenyl)-1 H-pyrazole-3-carboxamide 59}

a) Prepared from N-(4-fluorophenyl)-Z-3-chloro-2-(benzylsulfinyl)propenamide 7 and trimethylsilyldiazomethane

An ethereal solution of trimethylsilyldiazomethane ( $1.51 \mathrm{~mL}$ of a $2 \mathrm{M}$ solution, $3.0 \mathrm{mmol})$ was added to a stirring solution of $N$-(4-fluorophenyl)-Z-3-chloro-2(benzylsulfinyl)propenamide $7(0.20 \mathrm{~g}, 0.6 \mathrm{mmol})$ in ether $(25 \mathrm{~mL})$ and acetone $(1.5 \mathrm{~mL})$. Following stirring at room temperature for $6 \mathrm{~h}$ under a nitrogen atmosphere in the dark, the solvent and excess trimethylsilyldiazomethane were removed by concentration under reduced pressure to give the crude product as a yellow solid. Following purification by column chromatography on silica gel using hexane-ethyl acetate (gradient elution 20-40 $\%$ ethyl acetate), 59 was obtained as a white solid ( $0.07 \mathrm{~g}, 58 \%), \mathrm{mp} 151-153{ }^{\circ} \mathrm{C}$; (Found C, 58.44; H, 4.01; N, 19.52; F, 9.64. $\mathrm{C}_{10} \mathrm{H}_{8} \mathrm{FN}_{3} \mathrm{O}$ requires $\mathrm{C}, 58.53 ; \mathrm{H}, 3.93 ; \mathrm{N}, 20.48 ; \mathrm{F}$, 9.26\%); $v_{\max } / \mathrm{cm}^{-1}(\mathrm{KBr}) 3180(\mathrm{NH}), 2959(\mathrm{CH}), 1656(\mathrm{CO}), 1567,1508,1321 ; \delta_{\mathrm{H}}(300$ MHz, DMSO-d $\left.d_{6}\right) 6.79[1 \mathrm{H}$, br s, C(4)H], 7.09-7.27 (2H, m, ArH), 7.73-7.97 [3H, m, $\mathrm{C}(5) H \& \operatorname{Ar} H], 10.13\left(1 \mathrm{H}\right.$, br s, $\mathrm{N} H$ of carboxamide), $13.41\left[1 \mathrm{H}\right.$, br s, N(1)H]; $\delta_{\mathrm{C}}(75.5$ MHz, DMSO- $\left.d_{6}\right) 106.0[\mathrm{CH}, C(4) \mathrm{H}], 115.4\left[\mathrm{CH}, \mathrm{d},{ }^{2} J_{\mathrm{CF}} 22\right.$, aromatic $\left.C\left(3^{\prime}\right) \mathrm{H}\right], 122.3$ $\left[\mathrm{CH}, \mathrm{d},{ }^{3} J_{\mathrm{CF}} 8\right.$, aromatic $\left.C\left(2^{\prime}\right) \mathrm{H}\right], 130.8[\mathrm{CH}, C(5) \mathrm{H}], 135.6(\mathrm{C}$, aromatic $C), 146.9[\mathrm{C}$, $C(3)], 158.5$ [C, d, ${ }^{1} J_{\mathrm{CF}} 239$, aromatic $\left.C\left(4^{\prime}\right)\right], 160.8$ (C, CO); HRMS (ES+): Exact mass calculated for $\mathrm{C}_{10} \mathrm{H}_{9} \mathrm{~N}_{3} \mathrm{OF}[\mathrm{M}+\mathrm{H}]^{+}, 206.0730$. Found 206.0729; m/z (ES+) 206.2 $\left\{\left[\left(\mathrm{C}_{10} \mathrm{H}_{8} \mathrm{~N}_{3} \mathrm{OF}\right)+\mathrm{H}^{+}\right], 100 \%\right\}$. 
b) Prepared from N-(4-fluorophenyl)-Z-3-chloro-2-(benzenesulfinyl)propenamide $\mathbf{1 3}$ and trimethylsilyldiazomethane

The title compound was also synthesised using an ethereal solution of trimethylsilyldiazomethane $(1.54 \mathrm{~mL}$ of a $2 \mathrm{M}$ solution, $3.1 \mathrm{mmol})$ and $\mathrm{N}$-(4fluorophenyl)-Z-3-chloro-2-(benzenesulfinyl)propenamide 13 (0.20 g, $0.6 \mathrm{mmol})$ in ether $(20 \mathrm{~mL})$ under nitrogen at room temperature in the dark. The reaction solution was then stirred for $6 \mathrm{~h}$. The solvent and excess trimethylsilyldiazomethane were removed by evaporation at reduced pressure to give the crude product as a yellow solid. Purification by column chromatography using hexane-ethyl acetate (gradient elution 20-60\% ethyl acetate) gave 59 as a white solid $\left(0.02\right.$ g, 16\%), with IR and ${ }^{1} \mathrm{H}$ NMR spectroscopic details identical to above.

\section{$N$-n-Butyl-1H-pyrazole-3-carboxamide 60}

b) Prepared from N-n-butyl-Z-3-chloro-2-(benzylsulfinyl)propenamide 11 and trimethylsilyldiazomethane

An ethereal solution of trimethylsilyldiazomethane $(2.41 \mathrm{~mL}$ of a $2 \mathrm{M}$ solution, $4.8 \mathrm{mmol})$ was added to a stirring solution of $N$ - $n$-butyl-Z-3-chloro-2-(benzylsulfinyl)propenamide $11(0.29 \mathrm{~g}, 1.0 \mathrm{mmol})$ in ether $(25 \mathrm{~mL})$ under nitrogen at room temperature in the dark. The reaction solution was then stirred for $6 \mathrm{~h}$. The solvent and excess trimethylsilyldiazomethane were removed by evaporation at reduced pressure to give the crude product as a yellow oil. Purification by column chromatography using hexane-ethyl acetate (gradient elution $20-80 \%$ ethyl acetate) gave $\mathbf{6 0}$ as a low melting white solid (0.08 g, 52\%); (Found C, 57.69; H, 7.88; N, 24.56. $\mathrm{C}_{8} \mathrm{H}_{13} \mathrm{~N}_{3} \mathrm{O}$ requires $\mathrm{C}, 57.46 ; \mathrm{H}, 7.84 ; \mathrm{N}$, 25.13\%); $v_{\max } / \mathrm{cm}^{-1}(\mathrm{KBr}) 3292(\mathrm{NH}), 3135(\mathrm{CH}), 2955(\mathrm{CH}), 1641(\mathrm{CO}), 1559,1313 ; \delta_{\mathrm{H}}$ $\left(300 \mathrm{MHz}, \mathrm{DMSO}-d_{6}\right) 0.89$ [3H, t, $J$ 7.2, C(4') $\left.H_{3}\right], 1.30$ [2H, sextet, $J$ 7.2, C(3') $H_{2}$ ], 1.48 [2H, quintet, $\left.J 7.2, \mathrm{C}\left(2^{\prime}\right) H_{2}\right], 3.23\left[2 \mathrm{H}, \mathrm{dt}, J 6.9,6.9, \mathrm{NHCH}_{2}\right], 6.66[1 \mathrm{H}$, br s, C(4)H], $7.74[1 \mathrm{H}$, br s, $\mathrm{C}(5) H], 8.12(1 \mathrm{H}$, br s, $\mathrm{N} H$ of carboxamide $), 13.18[1 \mathrm{H}$, br s, $\mathrm{N}(1) H]$; $\delta_{\mathrm{C}}$ (75.5 MHz, DMSO- $\left.d_{6}\right) 13.7\left[\mathrm{CH}_{3}, C\left(4^{\prime}\right) \mathrm{H}_{3}\right], 19.6\left[\mathrm{CH}_{2}, C\left(3^{\prime}\right) \mathrm{H}_{2}\right], 31.4\left[\mathrm{CH}_{2}, C\left(2^{\prime}\right) \mathrm{H}_{2}\right]$, $38.0\left(\mathrm{CH}_{2}, \mathrm{NHCH}_{2}\right), 104.8[\mathrm{CH}, C(4) \mathrm{H}], 129.8[\mathrm{CH}, C(5) \mathrm{H}], 146.8[\mathrm{C}, C(3)], 161.6(\mathrm{C}$, CO); HRMS (ES+): Exact mass calculated for $\mathrm{C}_{8} \mathrm{H}_{14} \mathrm{~N}_{3} \mathrm{O}[\mathrm{M}+\mathrm{H}]^{+}, 168.1137$. Found $168.1138 ; \mathrm{m} / \mathrm{z}(\mathrm{ES}+) 168.2\left\{\left[\left(\mathrm{C}_{8} \mathrm{H}_{13} \mathrm{~N}_{3} \mathrm{O}\right)+\mathrm{H}^{+}\right], 100 \%\right\}$. 
b) Prepared from N-n-butyl-Z-3-chloro-2-(benzenesulfinyl)propenamide 17 and trimethylsilyldiazomethane

The title compound was also prepared using an excess of an ethereal solution of trimethylsilyldiazomethane $(1.85 \mathrm{~mL}$ of a $2 \mathrm{M}$ solution, $3.7 \mathrm{mmol})$ and $N$ - $n$-butyl-Z-3chloro-2-(benzenesulfinyl)propenamide $17(0.21 \mathrm{~g}, 0.7 \mathrm{mmol})$ in ether $(20 \mathrm{~mL})$. Following stirring at room temperature for $6 \mathrm{~h}$ and removal of the solvent and excess trimethylsilyldiazomethane, the crude product was obtained as a yellow oil. After purification by column chromatography using hexane-ethyl acetate (gradient elution 20$80 \%$ ethyl acetate), $\mathbf{6 0}$ was obtained as a low melting white solid ( $0.03 \mathrm{~g}, 28 \%)$, with IR and ${ }^{1} \mathrm{H}$ NMR spectroscopic details identical to above.

\section{$N$-Methyl-1 $H$-pyrazole-3-carboxamide 61}

An ethereal solution of trimethylsilyldiazomethane ( $2.56 \mathrm{~mL}$ of a $2 \mathrm{M}$ solution, $5.1 \mathrm{mmol})$ was added to a stirring solution of $N$-methyl-Z-3-chloro-2-(benzylsulfinyl)propenamide 9 $(0.26 \mathrm{~g}, 1.0 \mathrm{mmol})$ in ether $(25 \mathrm{~mL})$ under nitrogen at room temperature in the dark. The reaction solution was then stirred for $6 \mathrm{~h}$. The solvent and excess trimethylsilyldiazomethane were removed by evaporation at reduced pressure to give the crude product as a yellow oil. Purification by column chromatography using hexane-ethyl acetate (gradient elution $20-80 \%$ ethyl acetate) gave 61 as a white solid (0.09 g, 73\%), mp 128-129 ${ }^{\circ} \mathrm{C} ; v_{\max } / \mathrm{cm}^{-1}(\mathrm{KBr}) 3294(\mathrm{NH}), 1648(\mathrm{CO}), 1565,1311 ; \delta_{\mathrm{H}}(300 \mathrm{MHz}$, DMSO- $\left.d_{6}\right) 2.76\left(3 \mathrm{H}, \mathrm{d}, J 4.8, \mathrm{NHCH}_{3}\right), 6.66[1 \mathrm{H}, \mathrm{br} \mathrm{d}, J 2.4, \mathrm{C}(4) H], 7.76[1 \mathrm{H}, \mathrm{br} \mathrm{d}, J$ $2.1, \mathrm{C}(5) H], 8.16\left(1 \mathrm{H}\right.$, br s, $\mathrm{N} H$ of carboxamide); $\delta_{\mathrm{C}}\left(75.5 \mathrm{MHz}, \mathrm{DMSO}-d_{6}\right) 25.5\left(\mathrm{CH}_{3}\right.$, $\left.\mathrm{NHCH}_{3}\right), 104.7[\mathrm{CH}, C(4) \mathrm{H}], 131.5[\mathrm{CH}, \mathrm{br}, C(5) \mathrm{H}], 145.3[\mathrm{C}, \mathrm{br}, C(3)], 161.7(\mathrm{C}, \mathrm{br}$, CO); HRMS (ES+): Exact mass calculated for $\mathrm{C}_{5} \mathrm{H}_{8} \mathrm{~N}_{3} \mathrm{O}[\mathrm{M}+\mathrm{H}]^{+}, 126.0667$. Found 126.0668; m/z (ES+) $126.0\left\{\left[\left(\mathrm{C}_{5} \mathrm{H}_{7} \mathrm{~N}_{3} \mathrm{O}\right)+\mathrm{H}^{+}\right], 100 \%\right\}$.

\section{$\left(3 R^{*}, 4 R^{*}, S_{\mathrm{S}}^{*}\right)-N$-Benzyl-3-(benzylsulfinyl)-4-chloro-4,5-dihydro-3H-pyrazole-3-}

\section{carboxamide 67}

A solution of $N$-benzyl-Z-3-chloro-2-(benzylsulfinyl)propenamide 10 (0.18 g, $0.5 \mathrm{mmol})$ in ether $(15 \mathrm{~mL})$ and acetone $(2 \mathrm{~mL})$ was added to an excess of an ethereal solution of diazomethane [prepared from Diazald $\left.{ }^{\circledR}(0.84 \mathrm{~g}, 3.9 \mathrm{mmol})\right]$ cooled in an ice-salt bath 
while stirring. The solution was allowed to return slowly to room temperature while stirring for $4 \mathrm{~h}$. A precipitate formed as the reaction progressed. The product was collected by filtration through a sintered glass funnel (grade 3 ) to give $\mathbf{6 7}$ as a white solid $(0.13 \mathrm{~g}, 62 \%)$ as a single diastereomer, $\mathrm{mp} 87-88^{\circ} \mathrm{C}$; (Found $\mathrm{C}, 57.49, \mathrm{H}, 4.93, \mathrm{~N}, 10.95$, $\mathrm{S}, 8.76, \mathrm{Cl}, 9.20 ; \mathrm{C}_{18} \mathrm{H}_{18} \mathrm{ClN}_{3} \mathrm{O}_{2} \mathrm{~S}$ requires $\mathrm{C}, 57.52, \mathrm{H}, 4.83, \mathrm{~N}, 11.18, \mathrm{~S}, 8.53, \mathrm{Cl}$, 9.43\%); $v_{\max } / \mathrm{cm}^{-1}(\mathrm{KBr}) 3326(\mathrm{NH}), 3030(\mathrm{CH}), 2976(\mathrm{CH}), 1666(\mathrm{CO}), 1541(\mathrm{~N}=\mathrm{N})$, 1496, 1455, 1265, $1035(\mathrm{SO}) ; \delta_{\mathrm{H}}\left(300 \mathrm{MHz}, \mathrm{CDCl}_{3}\right) 3.87(1 \mathrm{H}, \mathrm{d}, \mathrm{A}$ of AB system, $J$ 12.6, $\left.\mathrm{SCH}_{2}\right), 4.26\left(1 \mathrm{H}, \mathrm{d}, \mathrm{B}\right.$ of $\mathrm{AB}$ system, $\left.J 12.9, \mathrm{SC} H_{2}\right), 4.41\left(1 \mathrm{H}, \mathrm{dd}, \mathrm{A}\right.$ of $\mathrm{ABX}, J_{\mathrm{AB}}$ $\left.14.7, J_{\mathrm{AX}} 5.4, \mathrm{NHCH}_{A} \mathrm{H}_{\mathrm{B}}\right), 4.62\left(1 \mathrm{H}, \mathrm{dd}, \mathrm{B}\right.$ of $\left.\mathrm{ABX}, J_{\mathrm{AB}} 14.9, J_{\mathrm{BX}} 6.6, \mathrm{NHCH}_{\mathrm{A}} H_{B}\right), 4.83-$ $4.93\left[1 \mathrm{H}, \mathrm{m}\right.$, one of $\left.\mathrm{C}(5) \mathrm{H}_{2}\right], 5.16-5.25\left[2 \mathrm{H}, \mathrm{m}, \mathrm{C}(4) \mathrm{H}\right.$ \& one of $\left.\mathrm{C}(5) \mathrm{H}_{2}\right], 7.16-7.40(10 \mathrm{H}$, m, $\operatorname{ArH}), 7.71(1 \mathrm{H}$, br s, $\mathrm{NH}) ; \delta_{\mathrm{C}}\left(75.5 \mathrm{MHz}, \mathrm{CDCl}_{3}\right) 44.4\left(\mathrm{CH}_{2}, \mathrm{NHCH}_{2}\right), 51.8[\mathrm{CH}$, $C(4) \mathrm{H}], 56.5\left(\mathrm{CH}_{2}, \mathrm{SCH}_{2}\right), 87.4\left[\mathrm{CH}_{2}, C(5) \mathrm{H}_{2}\right], 108.6[\mathrm{C}, C(3)], 127.9,128.0,128.88$, 128.91, $129.1(5 \times \mathrm{CH}, 5 \times$ aromatic $\mathrm{CH}), 129.6(\mathrm{C}$, aromatic $\mathrm{C}), 130.4(\mathrm{CH}$, aromatic CH), 137.0 (C, aromatic C), 159.9 (C, CO); HRMS (ES+): Exact mass calculated for $\mathrm{C}_{18} \mathrm{H}_{19} \mathrm{NO}_{2} \mathrm{~S}^{35} \mathrm{Cl}\left[(\mathrm{M}+\mathrm{H})^{+}-\mathrm{N}_{2}\right], 348.0825$. Found $348.0815 ; \mathrm{m} / \mathrm{z}(\mathrm{ES}+) 350.1$ $\left\{\left[\left(\mathrm{C}_{18} \mathrm{H}_{18} \mathrm{NO}_{2} \mathrm{~S}^{37} \mathrm{Cl}\right)+\mathrm{H}^{+}\right], 4 \%\right\}, 348.1\left\{\left[\left(\mathrm{C}_{18} \mathrm{H}_{18} \mathrm{NO}_{2} \mathrm{~S}^{35} \mathrm{Cl}\right)+\mathrm{H}^{+}\right], 10 \%\right\}, 87.9(100 \%)$.

\section{$\left(3 R^{*}, 4 R^{*}, S_{\mathrm{S}}^{*}\right)-3-(B e n z y l s u l f i n y l)-4-c h l o r o-4,5-d i h y d r o-N$-methyl-3H-pyrazole-3-}

\section{carboxamide $68 \& N$-Methyl-1 $H$-pyrazole-3-carboxamide 61}

A solution of $N$-methyl-Z-3-chloro-2-(benzylsulfinyl)propenamide 9 (0.15 g, $0.6 \mathrm{mmol})$ in ether $(15 \mathrm{~mL})$ and acetone $(2 \mathrm{~mL})$ was added to an excess of an ethereal solution of diazomethane [prepared from Diazald $\left.{ }^{\circledR}(0.84 \mathrm{~g}, 3.9 \mathrm{mmol})\right]$ cooled in an ice-salt bath while stirring. The solution was allowed to return slowly to room temperature while stirring for $4 \mathrm{~h}$. A precipitate formed as the reaction progressed. The product was collected by filtration through a sintered glass funnel (grade 3) to give $\mathbf{6 8}$ as a white solid (0.10 g, 55\%), mp 58-59 ${ }^{\circ} \mathrm{C} ; v_{\max } / \mathrm{cm}^{-1}$ (KBr) $3342(\mathrm{NH}), 3052(\mathrm{CH}), 2921(\mathrm{CH}), 1671$ $(\mathrm{CO}), 1550(\mathrm{~N}=\mathrm{N}), 1039(\mathrm{SO}) ; \delta_{\mathrm{H}}\left(300 \mathrm{MHz}, \mathrm{CDCl}_{3}\right) 2.93\left(3 \mathrm{H}, \mathrm{d}, J 4.8, \mathrm{NHCH}_{3}\right), 4.02$ $\left(1 \mathrm{H}, \mathrm{d}, \mathrm{A}\right.$ of AB system, $\left.J 12.9, \mathrm{SCH}_{2}\right), 4.41\left(1 \mathrm{H}, \mathrm{d}, \mathrm{B}\right.$ of AB system, $\left.J 12.9, \mathrm{SCH}_{2}\right)$, 4.83-4.92 [1H, m, one of $\left.\mathrm{C}(5) H_{2}\right], 5.18-5.27$ [2H, m, C(4)H \& one of C $\left.(5) H_{2}\right], 7.15(1 \mathrm{H}$, br s, $\mathrm{NH}), 7.24-7.43(5 \mathrm{H}, \mathrm{m}, \mathrm{Ar} H)$; In DMSO- $d_{6} \mathbf{6 8}$ decomposed rapidly to give the pyrazole 61 with characteristic peaks at $\delta_{\mathrm{H}}\left(300 \mathrm{MHz}, \mathrm{DMSO}-d_{6}\right) 2.75\left(3 \mathrm{H}, \mathrm{s}, \mathrm{NHCH}_{3}\right)$, 
$6.66[1 \mathrm{H}, \mathrm{d}, J 2.1, \mathrm{C}(4) H], 7.27-7.50(5 \mathrm{H}, \mathrm{m}, \mathrm{Ar} H), 7.75[1 \mathrm{H}, \mathrm{d}, J 2.1, \mathrm{C}(5) H], 8.15(1 \mathrm{H}$, br s, $\mathrm{N} H) ; \delta_{\mathrm{C}}\left(75.5 \mathrm{MHz}, \mathrm{DMSO}-d_{6}\right) 25.5\left[\mathrm{CH}_{3}, \mathrm{NHCH}_{3}\right], 104.7[\mathrm{CH}, C(4) \mathrm{H}], 131.5$ [CH, C(5)H], 144.8 [C, C(3)], $161.7(\mathrm{C}, \mathrm{CO})$; HRMS (ES+): Exact mass calculated for $\mathrm{C}_{12} \mathrm{H}_{15} \mathrm{NO}_{2} \mathrm{~S}^{35} \mathrm{Cl}\left[(\mathrm{M}+\mathrm{H})^{+}-\mathrm{N}_{2}\right], 272.0512$. Found 272.0520; m/z (ES+) 300.1 $\left\{\left[\left(\mathrm{C}_{12} \mathrm{H}_{14} \mathrm{~N}_{3} \mathrm{O}_{2} \mathrm{~S}^{35} \mathrm{Cl}\right)+\mathrm{H}^{+}\right], 8 \%\right\}, 348.1\left\{\left[\left(\mathrm{C}_{12} \mathrm{H}_{14} \mathrm{NO}_{2} \mathrm{~S}^{35} \mathrm{Cl}\right)+\mathrm{H}^{+}\right], 6 \%\right\}, 87.9(100 \%)$.

$\left(3 R^{*}, 4 R^{*}, S_{\mathrm{S}}^{*}\right)-3$-(n-Butylsulfinyl)-4,5-dihydro- $N$-benzyl-3H-pyrazole-3-carboxamide $69 \& N$-benzyl-1 $H$-pyrazole-3-carboxamide 58

A solution of $N$-benzyl-Z-3-chloro-2-( $n$-butylsulfinyl)propenamide 27 (0.17 g, $0.6 \mathrm{mmol})$ in ether $(15 \mathrm{~mL})$ was added to an excess of an ethereal solution of diazomethane [prepared from Diazald $\left.{ }^{\circledR}(0.84 \mathrm{~g}, 3.9 \mathrm{mmol})\right]$ cooled in an ice-salt bath while stirring. The solution was allowed to return slowly to room temperature while stirring for $4 \mathrm{~h}$. A precipitate formed as the reaction progressed. Following filtration of the reaction mixture through a sintered glass funnel (grade 3), 69 was obtained as a low melting white solid $(0.15 \mathrm{~g}, 77 \%)$ as a single diastereomer. The ${ }^{1} \mathrm{H}$ NMR spectrum of the crude product was very clean; $v_{\max } / \mathrm{cm}^{-1}(\mathrm{KBr}) 3328(\mathrm{NH}), 3035(\mathrm{CH}), 2932(\mathrm{CH}), 1671(\mathrm{CO}), 1540(\mathrm{~N}=\mathrm{N})$, $1046(\mathrm{SO}) ; \delta_{\mathrm{H}}\left(300 \mathrm{MHz}, \mathrm{CDCl}_{3}\right) 0.94\left[3 \mathrm{H}, \mathrm{t}, J 7.5, \mathrm{C}\left(4^{\prime}\right) H_{3}\right], 1.33-1.56[2 \mathrm{H}, \mathrm{m}$, $\left.\mathrm{C}\left(3^{\prime}\right) H_{2}\right], 1.74-1.92$ [2H, m, C $\left(2^{\prime}\right) H_{2}$ ], $2.82\left(1 \mathrm{H}, \mathrm{ddd}, J 12.6,8.4,6.0\right.$, one of $\left.\mathrm{SCH}_{2}\right), 2.92$ $\left(1 \mathrm{H}, \mathrm{ddd}, J 12.6,8.7,7.8\right.$, one of $\left.\mathrm{SCH}_{2}\right), 4.40\left(1 \mathrm{H}, \mathrm{dd}, \mathrm{A}\right.$ of $\mathrm{ABX}, J_{\mathrm{AB}} 15.0, J_{\mathrm{AX}} 5.7$, one of $\left.\mathrm{NCH}_{2}\right), 4.54\left(1 \mathrm{H}, \mathrm{B}\right.$ of $\mathrm{ABX}, J_{\mathrm{AB}} 14.7, J_{\mathrm{BX}} 6.3$, one of $\left.\mathrm{NCH}_{2}\right), 4.86$ [1H, A of ABX, $J_{\mathrm{AB}} 18.9, J_{\mathrm{AX}} 6.0$, one of $\left.\mathrm{C}(5) H_{2}\right], 5.06[1 \mathrm{H}, \mathrm{dd}, J 6.0,1.8, \mathrm{C}(4) H], 5.17$ [1H, B of ABX, $J_{\mathrm{AB}} 18.9, J_{\mathrm{BX}} 1.8$, one of $\left.\mathrm{C}(5) H_{2}\right], 7.22-7.40(5 \mathrm{H}, \mathrm{m}, \mathrm{Ar} H), 7.68(1 \mathrm{H}$, br s, NH).

In DMSO- $d_{6} 68$ decomposed rapidly to give the pyrazole 58 with characteristic peaks at $\delta_{\mathrm{H}}\left(300 \mathrm{MHz}, \mathrm{DMSO}-d_{6}\right) 4.44\left(2 \mathrm{H}, \mathrm{d}, J 6.3, \mathrm{NHCH}_{2}\right), 6.71[1 \mathrm{H}, \mathrm{d}, J 2.4, \mathrm{C}(4) H], 7.78$ $[1 \mathrm{H}, \mathrm{d}, J 2.1, \mathrm{C}(5) H], 8.78(1 \mathrm{H}, \mathrm{br} \mathrm{t}, J 6.0, \mathrm{NH}$ of carboxamide), a broad signal was also observed at $5.38 \mathrm{ppm}$ (water signal normally observed at $3.40 \mathrm{ppm}) ; \delta_{\mathrm{C}}(75.5 \mathrm{MHz}$, DMSO- $\left.d_{6}\right) 42.2\left(\mathrm{CH}_{2}, \mathrm{NHCH}_{2}\right), 105.4[\mathrm{CH}, C(4) \mathrm{H}], 127.0,127.6,128.6,130.3[4 \times \mathrm{CH}$, aromatic $C \mathrm{H} \& C(5) \mathrm{H}], 140.3(\mathrm{C}$, aromatic $C), 146.9[\mathrm{C}, C(3)], 162.2(\mathrm{C}, C O) ; \mathrm{m} / \mathrm{z}$ (ES+) $202.0\left\{\left[\left(\mathrm{C}_{11} \mathrm{H}_{11} \mathrm{~N}_{3} \mathrm{O}\right)+\mathrm{H}^{+}\right], 100 \%\right\}$ (eliminated pyrazole). 
$\left(3 R^{*}, 4 R^{*}\right)-3-(B e n z y l t h i o)-4-c h l o r o-N-(4-f l u o r o p h e n y l)-4,5-d i h y d r o-3 H$-pyrazole-3carboxamide 70

A solution of $N$-(4-fluorophenyl)-Z-3-chloro-2-(benzylthio)propenamide 19 (0.18 g, 0.6 $\mathrm{mmol})$ in ether $(15 \mathrm{~mL})$ was added to an excess of an ethereal solution of diazomethane [prepared from Diazald $\left.{ }^{\circledR}(0.84 \mathrm{~g}, 3.9 \mathrm{mmol})\right]$ cooled in an ice-salt bath while stirring. The solution was allowed to return slowly to room temperature while stirring for $6 \mathrm{~h}$. Following removal of the solvent and excess diazomethane by evaporation under reduced pressure, 70 was obtained as a white solid $(0.17 \mathrm{~g}, 79 \%)$ as a single diastereomer (the ${ }^{1} \mathrm{H}$ NMR spectrum of the crude product was very clean), mp $69-70{ }^{\circ} \mathrm{C}$; (Found $\mathrm{C}, 56.63 ; \mathrm{H}$, $4.11 ; \mathrm{N}, 11.58 ; \mathrm{Cl}, 10.40 . \mathrm{C}_{17} \mathrm{H}_{15} \mathrm{ClN}_{3} \mathrm{OSF}$ requires $\mathrm{C}, 56.12 ; \mathrm{H}, 4.16 ; \mathrm{N}, 11.55 ; \mathrm{Cl}$, 9.74\%); $v_{\max } / \mathrm{cm}^{-1}(\mathrm{KBr}) 3268(\mathrm{NH}), 3021(\mathrm{CH}), 1668(\mathrm{CO}), 1508(\mathrm{~N}=\mathrm{N}) ; \delta_{\mathrm{H}}(300 \mathrm{MHz}$, $\left.\mathrm{CDCl}_{3}\right) 4.09\left(1 \mathrm{H}, \mathrm{d}, \mathrm{A}\right.$ of $\mathrm{AB}$ system, $J_{\mathrm{AB}} 12.0$, one of $\left.\mathrm{SCH}_{2}\right), 4.13(1 \mathrm{H}, \mathrm{d}, \mathrm{B}$ of $\mathrm{AB}$ system, $J_{\mathrm{AB}} 12.0$, one of $\left.\mathrm{SCH}_{2}\right), 4.90\left[1 \mathrm{H}, \mathrm{dd}, \mathrm{C}\right.$ of $\left.\mathrm{ABC}, J_{\mathrm{AC}} 6.6, J_{\mathrm{BC}} 4.2, \mathrm{C}(4) H\right], 4.96$ $\left[1 \mathrm{H}, \mathrm{A}\right.$ of $\mathrm{ABC}, J_{\mathrm{AB}} 18.8, J_{\mathrm{AC}} 4.2$, one of $\left.\mathrm{C}(5) H_{2}\right], 5.04$ [1H, B of ABC, $J_{\mathrm{AB}} 18.8, J_{\mathrm{BC}} 6.6$, one of $\left.\mathrm{C}(5) H_{2}\right], 6.97-7.07(2 \mathrm{H}, \mathrm{m}, \operatorname{Ar} H), 7.17-7.45(7 \mathrm{H}, \mathrm{m}, \operatorname{Ar} H), 8.54\left(1 \mathrm{H}, \mathrm{br}\right.$ s, NH); $\delta_{\mathrm{C}}$ (75.5 MHz, DMSO-d $\left.d_{6}\right) 34.8\left(\mathrm{CH}_{2}, \mathrm{SCH}_{2}\right), 57.0[\mathrm{CH}, C(4) \mathrm{H}], 84.2\left[\mathrm{CH}_{2}, C(5) \mathrm{H}_{2}\right], 100.1$ $[\mathrm{C}, C(3)], 115.1\left[\mathrm{CH}, \mathrm{d},{ }^{2} J_{\mathrm{CF}} 22\right.$, aromatic $\left.C\left(3^{\prime}\right) \mathrm{H}\right], 123.2\left[\mathrm{CH}, \mathrm{d},{ }^{3} J_{\mathrm{CF}} 8\right.$, aromatic $\left.C\left(2^{\prime}\right) \mathrm{H}\right], 127.4,128.6,129.2(3 \times \mathrm{CH}, 3 \times$ aromatic $\mathrm{CH}), 134.0,135.9(2 \times \mathrm{C}, 2 \times$ aromatic $C$ ), 158.8 [C, d, ${ }^{1} J_{\mathrm{CF}} 242$, aromatic $\left.C\left(4^{\prime}\right)\right], 163.7$ (C, CO); HRMS (ES+): Exact mass calculated for $\mathrm{C}_{17} \mathrm{H}_{15} \mathrm{~N}_{3} \mathrm{OSF}\left[(\mathrm{M}+\mathrm{H})^{+}-\mathrm{HCl}\right], 328.0920$. Found $328.0927 ; \mathrm{m} / \mathrm{z}$ $(\mathrm{ES}+) 328.2\left\{\left[\left(\mathrm{C}_{17} \mathrm{H}_{14} \mathrm{~N}_{3} \mathrm{OSF}\right)+\mathrm{H}^{+}\right], 34 \%\right\}, 87.9(100 \%)$.

\section{4-(Benzylthio)- $N$-(4-methylphenyl)-1H-pyrazole-3-carboxamide 62}

An ethereal solution of trimethylsilyldiazomethane (1.94 $\mathrm{mL}$ of a $2 \mathrm{M}$ solution, $3.9 \mathrm{mmol})$ was added to a stirring solution of $N$-(4-methylphenyl)-Z-3-chloro-2(benzylthio)propenamide $20(0.25 \mathrm{~g}, 0.8 \mathrm{mmol})$ in ether $(25 \mathrm{~mL})$ under nitrogen at room temperature in the dark. The reaction solution was then stirred at room temperature and the reaction progress was monitored by TLC analysis. After stirring for $5 \mathrm{~h}$, TLC analysis showed that a lot of starting material still remained and a further $1.94 \mathrm{~mL}$ of trimethylsilyldiazomethane solution was added. Following stirring for $24 \mathrm{~h}$, a further 1.94 $\mathrm{mL}$ of trimethylsilyldiazomethane solution was added to the reaction mixture. After 
stirring for $48 \mathrm{~h}$, TLC analysis indicated complete consumption of the starting material and the solvent and excess trimethylsilyldiazomethane were removed by evaporation at reduced pressure to give the crude product as an off-white solid. Purification by column chromatography using hexane-ethyl acetate (gradient elution 10-20\% ethyl acetate) gave 62 as a white solid $(0.16 \mathrm{~g}, 63 \%), \mathrm{mp} 150-151^{\circ} \mathrm{C}$; (Found C, 66.34; H, 5.39; N, 12.91; S, 10.18. $\mathrm{C}_{18} \mathrm{H}_{17} \mathrm{~N}_{3} \mathrm{OS}$ requires $\mathrm{C}, 66.85 ; \mathrm{H}, 5.30 ; \mathrm{N}, 12.99 ; \mathrm{S}, 9.91 \%$-C catalyst added); $v_{\max } / \mathrm{cm}^{-1}(\mathrm{KBr}) 3267(\mathrm{NH}), 3150(\mathrm{NH}), 3030(\mathrm{CH}), 2919(\mathrm{CH}), 1652(\mathrm{CO}), 1601,1551$, 1515,$1316 ; \delta_{\mathrm{H}}\left(300 \mathrm{MHz}, \mathrm{CDCl}_{3}\right) 2.35\left(3 \mathrm{H}, \mathrm{s}, \mathrm{ArCH}_{3}\right), 3.94\left(2 \mathrm{H}, \mathrm{s}, \mathrm{SCH} \mathrm{H}_{2}\right), 7.01-7.24$ $(7 \mathrm{H}, \mathrm{m}, \operatorname{Ar} H), 7.45(2 \mathrm{H}, \mathrm{d}, J$ 8.4, $\mathrm{Ar} H), 7.64[1 \mathrm{H}, \mathrm{s}, \mathrm{C}(5) H], 9.56(1 \mathrm{H}, \mathrm{br} \mathrm{s}, \mathrm{NH}$ of carboxamide), $12.40[1 \mathrm{H}$, br s, $\mathrm{N}(1) H] ; \delta_{\mathrm{C}}\left(75.5 \mathrm{MHz}, \mathrm{CDCl}_{3}\right) 21.0\left(\mathrm{CH}_{3}, \mathrm{ArCH}_{3}\right), 42.7$ $\left(\mathrm{CH}_{2}, \mathrm{SCH}_{2}\right), 107.9[\mathrm{C}, \mathrm{C}(4)], 120.2,127.7,128.71,128.73,129.6[4 \times \mathrm{CH}$, aromatic $C \mathrm{H}], 134.3,134.9,136.8[3 \times \mathrm{C}$, aromatic $C \& C(3)], 142.4[\mathrm{CH}, \mathrm{br}, C(5) \mathrm{H}], 157.7(\mathrm{C}$, $C O) ; \delta_{\mathrm{C}}\left(75.5 \mathrm{MHz}, \mathrm{DMSO}-d_{6}\right) 20.5\left(\mathrm{CH}_{3}, \mathrm{ArCH}_{3}\right), 38.0\left(\mathrm{CH}_{2}, \mathrm{SCH}_{2}\right), 113.3[\mathrm{C}, C(4)]$, $119.9,126.9,128.3,128.8,128.9(4 \times \mathrm{CH}$, aromatic $\mathrm{CH}), 132.3,136.2,137.8[3 \times \mathrm{C}$, aromatic $C \& C(3)], 143.2[\mathrm{CH}, \mathrm{br}, C(5) \mathrm{H}], 159.8(\mathrm{C}, C \mathrm{O})$; HRMS (ES+): Exact mass calculated for $\mathrm{C}_{18} \mathrm{H}_{18} \mathrm{~N}_{3} \mathrm{OS}[\mathrm{M}+\mathrm{H}]^{+}$, 324.1171. Found 324.1183; m/z (ES+) 324.2 $\left\{\left[\left(\mathrm{C}_{18} \mathrm{H}_{17} \mathrm{~N}_{3} \mathrm{OS}\right)+\mathrm{H}^{+}\right], 100 \%\right\}, 647.3\left\{\left[\left(\mathrm{C}_{36} \mathrm{H}_{34} \mathrm{~N}_{6} \mathrm{O}_{2} \mathrm{~S}_{2}\right)+\mathrm{H}^{+}\right], 22 \%\right\}$.

\section{$\mathrm{N}$-(4-Methylphenyl)-4-(phenylthio)-1H-pyrazole-3-carboxamide 64}

An ethereal solution of trimethylsilyldiazomethane $(1.80 \mathrm{~mL}$ of a $2 \mathrm{M}$ solution, $3.6 \mathrm{mmol})$ was added to a stirring solution of $N$-(4-methylphenyl)-Z-3-chloro-2(phenylthio)propenamide $24(0.22 \mathrm{~g}, 0.7 \mathrm{mmol})$ in ether $(20 \mathrm{~mL})$ under nitrogen at room temperature in the dark. The reaction solution was then stirred at room temperature and the reaction progress was monitored by TLC analysis. After stirring for $5 \mathrm{~h}$, TLC analysis showed that a lot of starting material still remained and a further $1.80 \mathrm{~mL}$ of trimethylsilyldiazomethane solution was added. Following stirring for $24 \mathrm{~h}$, a further 1.80 $\mathrm{mL}$ of trimethylsilyldiazomethane solution was added to the reaction mixture. After stirring for $48 \mathrm{~h}$, TLC analysis indicated complete consumption of the starting material and the solvent and excess trimethylsilyldiazomethane were removed by evaporation at reduced pressure to give the crude product as an off-white solid. Recrystallisation from ethyl acetate gave 64 as a white solid $(0.11 \mathrm{~g}, 50 \%)$, mp 181-183 ${ }^{\circ} \mathrm{C}$; (Found C, 65.64; H, 
4.88; N, 13.35; S, 10.46. $\mathrm{C}_{17} \mathrm{H}_{15} \mathrm{~N}_{3} \mathrm{OS}$ requires $\mathrm{C}, 66.00 ; \mathrm{H}, 4.89 ; \mathrm{N}, 13.58 ; \mathrm{S}, 10.36 \%$ ); $v_{\max } / \mathrm{cm}^{-1}(\mathrm{KBr}) 3298(\mathrm{NH}), 3120(\mathrm{NH}), 2919(\mathrm{CH}), 1683(\mathrm{CO}), 1603,1540,1314 ; \delta_{\mathrm{H}}$ $\left(300 \mathrm{MHz}, \mathrm{CDCl}_{3}\right) 2.32\left(3 \mathrm{H}, \mathrm{s}, \mathrm{ArCH}_{3}\right), 7.14(2 \mathrm{H}, \mathrm{d}, J$ 8.1, $\mathrm{ArH}), 7.17-7.34(5 \mathrm{H}, \mathrm{m}$, $\operatorname{Ar} H), 7.43(2 \mathrm{H}, \mathrm{d}, J$ 8.4, $\operatorname{Ar} H), 7.76[1 \mathrm{H}, \mathrm{s}, \mathrm{C}(5) H], 9.44(1 \mathrm{H}, \mathrm{br} \mathrm{s}, \mathrm{N} H$ of carboxamide), $11.33\left[1 \mathrm{H}\right.$, br s, N(1)H]; $\delta_{\mathrm{H}}\left(300 \mathrm{MHz}, \mathrm{DMSO}-d_{6}\right) 2.27(3 \mathrm{H}, \mathrm{s}, \mathrm{ArCH}), 7.06-7.35(7 \mathrm{H}$, m, $\operatorname{Ar} H), 7.65(2 \mathrm{H}, \mathrm{d}, J$ 8.1, $\operatorname{Ar} H), 7.98[1 \mathrm{H}, \mathrm{s}, \mathrm{C}(5) H], 10.06(1 \mathrm{H}$, br s, NH of carboxamide), $13.75[1 \mathrm{H}$, br s, $\mathrm{N}(1) H] ; \delta_{\mathrm{C}}\left(75.5 \mathrm{MHz}, \mathrm{DMSO}-d_{6}\right) 20.4\left(\mathrm{CH}_{3}, \mathrm{ArCH}_{3}\right)$, $108.7[\mathrm{C}, C(4)], 119.9,125.9,127.6,128.9,129.0[5 \times \mathrm{CH}$, aromatic $\mathrm{CH}$ or $C(3) \mathrm{H}]$, $132.3(\mathrm{C}$, aromatic $C), 134.7[\mathrm{CH}$, aromatic $\mathrm{CH}$ or $C(5) \mathrm{H}], 136.2,137.3(2 \times \mathrm{C}, 2 \times$ aromatic $C), 146.2$ [C, $C(3)], 159.6(\mathrm{C}, C \mathrm{O})$; HRMS (ES+): Exact mass calculated for

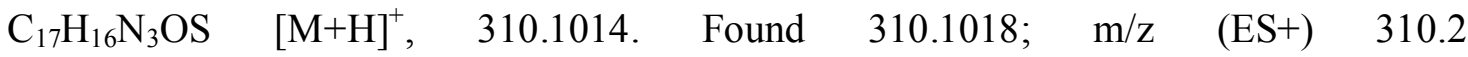
$\left\{\left[\left(\mathrm{C}_{17} \mathrm{H}_{15} \mathrm{~N}_{3} \mathrm{OS}\right)+\mathrm{H}^{+}\right], 100 \%\right\}, 619.3\left\{\left[\left(\mathrm{C}_{34} \mathrm{H}_{30} \mathrm{~N}_{6} \mathrm{O}_{2} \mathrm{~S}_{2}\right)+\mathrm{H}^{+}\right], 4 \%\right\}$.

\section{$\mathrm{N}$-Methyl-4-(phenylthio)-1 $\mathrm{H}$-pyrazole-3-carboxamide 65}

a) Prepared from N-methyl-Z-3-chloro-2-(benzenethio)propenamide $\mathbf{2 5}$ and trimethylsilyldiazomethane

An ethereal solution of trimethylsilyldiazomethane ( $2.62 \mathrm{~mL}$ of a $2 \mathrm{M}$ solution, $5.2 \mathrm{mmol})$ was added to a stirring solution of $\mathrm{N}$-methyl-Z-3-chloro-2-(phenylthio)propenamide $\mathbf{2 5}$ $(0.24 \mathrm{~g}, 1.1 \mathrm{mmol})$ in ether $(20 \mathrm{~mL})$ under nitrogen at room temperature in the dark. The reaction solution was then stirred at room temperature and the reaction progress was monitored by TLC analysis. After stirring for $5 \mathrm{~h}$, TLC analysis showed that a lot of starting material still remained and a further $2.62 \mathrm{~mL}$ of trimethylsilyldiazomethane solution was added. Following stirring for $24 \mathrm{~h}$, a further $2.62 \mathrm{~mL}$ of trimethylsilyldiazomethane solution was added to the reaction mixture. After stirring for $48 \mathrm{~h}$, TLC analysis indicated complete consumption of the starting material and the solvent and excess trimethylsilyldiazomethane were removed by evaporation at reduced pressure to give the crude product as an off-white solid. Recrystallisation from ethyl acetate gave $\mathbf{6 5}$ as a white solid $(0.15 \mathrm{~g}, 62 \%), \mathrm{mp} \mathrm{203-204}{ }^{\circ} \mathrm{C} ; v_{\max } / \mathrm{cm}^{-1}(\mathrm{KBr}) 3334$ (NH), $3111(\mathrm{NH}), 2920(\mathrm{CH}), 1649(\mathrm{CO})$, 1566, 1478, 1353; $\delta_{\mathrm{H}}\left(300 \mathrm{MHz}, \mathrm{CDCl}_{3}\right) 2.97$ $\left(3 \mathrm{H}, \mathrm{d}, J 5.1, \mathrm{NHCH}_{3}\right), 7.09-7.32(5 \mathrm{H}, \mathrm{m}, \mathrm{ArH}), 7.64(1 \mathrm{H}$, br s, $\mathrm{NH}$ of carboxamide), $7.72[1 \mathrm{H}, \mathrm{s}, \mathrm{C}(5) H], 11.64[1 \mathrm{H}, \mathrm{br} \mathrm{s}, \mathrm{N}(1) H] ; \delta_{\mathrm{H}}\left(300 \mathrm{MHz}, \mathrm{DMSO}-d_{6}\right) 2.73(3 \mathrm{H}, \mathrm{d}, J 4.5$, 
$\left.\mathrm{NHCH}_{3}\right), 7.12-7.38(5 \mathrm{H}, \mathrm{m}, \mathrm{Ar} H), 7.85[1 \mathrm{H}, \mathrm{s}, \mathrm{C}(5) H], 8.08(1 \mathrm{H}$, br d, $J 3.6, \mathrm{NH}$ of carboxamide), $13.56\left[1 \mathrm{H}\right.$, br s, N(1)H]; $\delta_{\mathrm{C}}\left(75.5 \mathrm{MHz}, \mathrm{DMSO}-d_{6}\right) 24.4\left(\mathrm{CH}_{3}, \mathrm{NHCH}_{3}\right)$, $106.9[\mathrm{C}, C(4)], 124.6,126.4,127.8,133.2($ br) $[4 \times \mathrm{CH}$, aromatic $C \mathrm{H} \& C(5) \mathrm{H}], 136.4$ (C, aromatic C), 144.8 [C, br, C(3)], 160.5 (C, CO); HRMS (ES+): Exact mass calculated for $\mathrm{C}_{11} \mathrm{H}_{12} \mathrm{~N}_{3} \mathrm{OS} \quad[\mathrm{M}+\mathrm{H}]^{+}, \quad 234.0701$. Found 234.0711; $\mathrm{m} / \mathrm{z} \quad(\mathrm{ES}+) \quad 234.1$ $\left\{\left[\left(\mathrm{C}_{11} \mathrm{H}_{11} \mathrm{~N}_{3} \mathrm{OS}\right)+\mathrm{H}^{+}\right], 100 \%\right\}, 467.2\left\{\left[\left(\mathrm{C}_{22} \mathrm{H}_{22} \mathrm{~N}_{6} \mathrm{O}_{2} \mathrm{~S}_{2}\right)+\mathrm{H}^{+}\right], 24 \%\right\}$.

b) Prepared from N-methyl-Z-3-chloro-2-(phenylthio)propenamide $\mathbf{2 5}$ and diazomethane The title compound was also prepared by addition of a solution of $N$-methyl-Z-3-chloro2-(benzenethio)propenamide $25(0.20 \mathrm{~g}, 0.9 \mathrm{mmol})$ in ether $(15 \mathrm{~mL})$ to an excess of an ethereal solution of diazomethane [prepared from Diazald $\left.{ }^{\circledR}(0.84 \mathrm{~g}, 3.9 \mathrm{mmol})\right]$ cooled in an ice-salt bath while stirring. The reaction mixture was allowed to return slowly to room temperature and then stirred for $4 \mathrm{~h}$. Following removal of the solvent and excess diazomethane, the crude product was obtained as a yellow solid. After purification by column chromatography on silica gel using hexane-ethyl acetate (gradient elution 20$40 \%$ ethyl acetate), $\mathbf{6 5}$ was obtained as a white solid (0.02 g, 10\%), with IR and ${ }^{1} \mathrm{H}$ NMR spectroscopic details identical to above.

\section{$\left(3 R^{*}, 4 R^{*}\right)-N$-Benzyl-3-(benzylthio)-4-chloro-4,5-dihydro-3H-pyrazole-3-}

\section{carboxamide $72 \& N$-benzyl-4-(benzylthio)-1 $H$-pyrazole-3-carboxamide 71}

A solution of $N$-benzyl-Z-3-chloro-2-(benzylthio)propenamide $28(0.18 \mathrm{~g}, 0.6 \mathrm{mmol})$ in ether $(15 \mathrm{~mL})$ was added to an excess of an ethereal solution of diazomethane [prepared from Diazald $\left.{ }^{\circledR}(0.84 \mathrm{~g}, 3.9 \mathrm{mmol})\right]$ cooled in an ice-salt bath while stirring. The solution was allowed to return slowly to room temperature while stirring for $6 \mathrm{~h}$. Following removal of the solvent and excess diazomethane by evaporation under reduced pressure, 72 was obtained as a white solid $(0.17 \mathrm{~g}, 84 \%)$ as a single diastereomer. The ${ }^{1} \mathrm{H}$ NMR spectrum of the crude product was very clean. \{Note: This compound decomposed before full analysis could be carried out, with characteristic signals for 71 seen at $\delta_{\mathrm{H}} 3.81$ (s, $\left.\left.\mathrm{SCH}_{2}\right), 4.47\left(\mathrm{~d}, J 6.3, \mathrm{NHCH}_{2}\right), 7.47[\mathrm{~s}, \mathrm{C}(5) H], 8.07(\mathrm{~s}, \mathrm{NH})\right\} ; \delta_{\mathrm{H}}\left(300 \mathrm{MHz}, \mathrm{CDCl}_{3}\right)$ $3.98\left(1 \mathrm{H}, \mathrm{d}, \mathrm{A}\right.$ of $\mathrm{AB}$ system, $J_{\mathrm{AB}} 11.7$, one of $\left.\mathrm{SCH}_{2}\right), 4.03\left(1 \mathrm{H}, \mathrm{d}, \mathrm{B}\right.$ of AB system, $J_{\mathrm{AB}}$ 11.7, one of $\left.\mathrm{SCH}_{2}\right), 4.35\left(1 \mathrm{H}, \mathrm{A}\right.$ of $\mathrm{ABX}, J_{\mathrm{AB}} 14.4, J_{\mathrm{AX}} 6.0$, one of $\left.\mathrm{NCH}_{2}\right), 4.45(1 \mathrm{H}, \mathrm{B}$ of 
$\mathrm{ABX}, J_{\mathrm{AB}} 14.4, J_{\mathrm{BX}} 6.0$, one of $\left.\mathrm{NCH}_{2}\right), 4.87$ [1H, dd, $\left.J 5.4,4.5, \mathrm{C}(4) H\right], 4.94-4.96[2 \mathrm{H}$, m, C(5)H $H_{2}$, $7.17(1 \mathrm{H}$, br s, NH), 7.22-7.42 (10H, m, ArH).

\section{$\left(3 R^{*}, 4 R^{*}\right)-N$-Benzyl-3-(phenylthio)-4-chloro-4,5-dihydro-3H-pyrazole-3-}

\section{carboxamide 73}

A solution of $N$-benzyl-Z-3-chloro-2-(phenylthio)propenamide $23(0.20 \mathrm{~g}, 0.7 \mathrm{mmol})$ in ether $(15 \mathrm{~mL})$ was added to an excess of an ethereal solution of diazomethane [prepared from Diazald $\left.{ }^{\circledR}(0.84 \mathrm{~g}, 3.9 \mathrm{mmol})\right]$ cooled in an ice-salt bath while stirring. The solution was allowed to return slowly to room temperature while stirring for $4 \mathrm{~h}$. A precipitate formed as the reaction progressed. Following filtration of the reaction mixture through a sintered glass funnel (grade 3), 73 was obtained as a white solid (0.19 g, 91\%), mp 87-89 ${ }^{\circ} \mathrm{C}$; (Found $\mathrm{C}, 59.04 ; \mathrm{H}, 4.55 ; \mathrm{N}, 11.79 ; \mathrm{Cl}, 10.27 ; \mathrm{S}, 8.93 . \mathrm{C}_{17} \mathrm{H}_{16} \mathrm{ClN}_{3} \mathrm{OS}$ requires $\mathrm{C}$, 59.04; H, 4.66; N, 12.15; Cl, 10.25; S, 9.27\%); $v_{\max } / \mathrm{cm}^{-1}(\mathrm{KBr}) 3321(\mathrm{NH}), 3014(\mathrm{CH})$, $1660(\mathrm{CO}), 1518(\mathrm{~N}=\mathrm{N}) ; \delta_{\mathrm{H}}\left(300 \mathrm{MHz}, \mathrm{CDCl}_{3}\right) 3.92\left(1 \mathrm{H}, \mathrm{dd}, \mathrm{A}\right.$ of $\mathrm{ABX}, J_{\mathrm{AB}} 14.6, J_{\mathrm{AX}}$ 5.4 one of $\left.\mathrm{NCH}_{2}\right), 4.20\left(1 \mathrm{H}, \mathrm{dd}, \mathrm{B}\right.$ of $\mathrm{ABX}, J_{\mathrm{AB}} 14.6, J_{\mathrm{BX}} 5.7$, one of $\left.\mathrm{NCH}_{2}\right), 4.72[1 \mathrm{H}$, dd, A of $\mathrm{ABC}, J_{\mathrm{AB}} 19.2, J_{\mathrm{AC}} 6.9$, one of C $\left.(5) H_{2}\right], 4.88\left[1 \mathrm{H}\right.$, dd, B of ABC, $J_{\mathrm{AB}} 19.2, J_{\mathrm{BC}}$ 3.3, one of C $\left.(5) H_{2}\right], 4.91\left[1 \mathrm{H}, \mathrm{dd}, \mathrm{C}\right.$ of $\left.\mathrm{ABC}, J_{\mathrm{AC}} 6.9, J_{\mathrm{BC}} 3.3, \mathrm{C}(4) H\right], 5.98(1 \mathrm{H}$, br s, $\mathrm{N} H), 6.86-6.93(2 \mathrm{H}, \mathrm{m}, \operatorname{Ar} H), 7.21-7.46(6 \mathrm{H}, \mathrm{m}, \operatorname{Ar} H), 7.68-7.75(2 \mathrm{H}, \mathrm{m}, \operatorname{Ar} H) ; \delta_{\mathrm{C}}(75.5$ MHz, DMSO-d $d_{6} 42.9\left(\mathrm{CH}_{2}, \mathrm{NHCH}_{2}\right), 57.3[\mathrm{CH}, C(4) \mathrm{H}], 83.7\left[\mathrm{CH}_{2}, C(5) \mathrm{H}_{2}\right], 101.5[\mathrm{C}$, $C(3)], 126.6,127.0,128.1,129.0,129.3(5 \times \mathrm{CH}, 5 \times$ aromatic $\mathrm{CH}), 129.5(\mathrm{C}$, aromatic C), $134.8(\mathrm{CH}$, aromatic $\mathrm{CH}), 138.3(\mathrm{C}$, aromatic $C), 164.1(\mathrm{C}, \mathrm{CO})$; HRMS (ES+): Exact mass calculated for $\mathrm{C}_{17} \mathrm{H}_{17} \mathrm{NOS}^{35} \mathrm{Cl}\left[(\mathrm{M}+\mathrm{H})^{+}-\mathrm{N}_{2}\right], 318.0719$. Found 318.0704; $\mathrm{m} / \mathrm{z}$ $(\mathrm{ES}+) 318.2\left\{\left[\left(\mathrm{C}_{17} \mathrm{H}_{16} \mathrm{NOS}^{35} \mathrm{Cl}\right)+\mathrm{H}^{+}\right], 8 \%\right\}, 87.9(100 \%)$.

\section{$\mathrm{N}$-Benzyl-4-(n-butylthio)-1 $\mathrm{H}$-pyrazole-3-carboxamide 74}

A solution of $N$-benzyl-Z-3-chloro-2-( $n$-butylthio)propenamide $92(0.18 \mathrm{~g}, 0.6 \mathrm{mmol})$ in ether $(15 \mathrm{~mL})$ was added to an excess of an ethereal solution of diazomethane [prepared from Diazald $\left.{ }^{\circledR}(0.84 \mathrm{~g}, 3.9 \mathrm{mmol})\right]$ cooled in an ice-salt bath while stirring. The reaction solution was allowed to return slowly to room temperature while stirring for $4 \mathrm{~h}$. Following removal of the solvent and excess diazomethane, the crude product was obtained as a pale yellow oil. After purification by column chromatography on silica gel 
using hexane-ethyl acetate (gradient elution $20-80 \%$ ethyl acetate), 74 was obtained as a white solid (0.07 g, 38\%), mp 120-122 ${ }^{\circ} \mathrm{C}$; (Found C, 61.66; H, 6.46; N, 14.24. $\mathrm{C}_{15} \mathrm{H}_{19} \mathrm{~N}_{3} \mathrm{OS}$ requires $\left.\mathrm{C}, 62.25 ; \mathrm{H}, 6.62 ; \mathrm{N}, 14.52 \%\right) ; v_{\max } / \mathrm{cm}^{-1}(\mathrm{KBr}) 3275(\mathrm{NH}), 3109$ $(\mathrm{NH}), 2951(\mathrm{CH}), 1644(\mathrm{CO}), 1555 ; \delta_{\mathrm{H}}\left(300 \mathrm{MHz}^{\mathrm{C}} \mathrm{CDCl}_{3}\right) 0.82\left[3 \mathrm{H}, \mathrm{t}, J\right.$ 7.5, $\left.\mathrm{C}\left(4^{\prime}\right) H_{3}\right]$, 1.21-1.36 [2H, m, C( $\left.3^{\prime}\right) H_{2}$ ], $1.37-1.57$ [2H, m, C(2') $\left.H_{2}\right], 2.63\left(2 \mathrm{H}, \mathrm{t}, J\right.$ 7.2, $\left.\mathrm{SCH}_{2}\right), 4.65$ $\left(2 \mathrm{H}, \mathrm{d}, J\right.$ 5.7, $\left.\mathrm{NHCH}_{2}\right), 7.22-7.39$ (5H, m, ArH), $7.62[1 \mathrm{H}, \mathrm{s}, \mathrm{C}(5) H], 8.35(1 \mathrm{H}$, br s, NH), $11.23[1 \mathrm{H}$, br s, $\mathrm{N}(1) H] ; \delta_{\mathrm{C}}\left(75.5 \mathrm{MHz}, \mathrm{CDCl}_{3}\right) 13.6\left[\mathrm{CH}_{3}, C\left(4^{\prime}\right) \mathrm{H}_{3}\right], 21.7\left[\mathrm{CH}_{2}\right.$, $\left.C\left(3^{\prime}\right) \mathrm{H}_{2}\right], 31.3\left[\mathrm{CH}_{2}, C\left(2^{\prime}\right) \mathrm{H}_{2}\right], 37.4\left(\mathrm{CH}_{2}, \mathrm{SCH}_{2}\right), 43.4\left(\mathrm{CH}_{2}, \mathrm{NHCH}_{2}\right), 109.2$ [C, $\left.C(4)\right]$, 127.6, 127.8, $128.7(3 \times \mathrm{CH}$, aromatic $\mathrm{CH}), 137.8(\mathrm{C}$, aromatic $\mathrm{C}), 140.2[\mathrm{CH}, \mathrm{br}$, $C(5) \mathrm{H}], 160.2(\mathrm{C}, C \mathrm{O})$; HRMS (ES+): Exact mass calculated for $\mathrm{C}_{15} \mathrm{H}_{20} \mathrm{~N}_{3} \mathrm{OS}[\mathrm{M}+\mathrm{H}]^{+}$, 290.1327. Found 290.1339; m/z (ES+) $290.2\left\{\left[\left(\mathrm{C}_{15} \mathrm{H}_{19} \mathrm{~N}_{3} \mathrm{OS}\right)+\mathrm{H}^{+}\right], 100 \%\right\}, 579.3$ $\left\{\left[\left(\mathrm{C}_{30} \mathrm{H}_{38} \mathrm{~N}_{6} \mathrm{O}_{2} \mathrm{~S}_{2}\right)+\mathrm{H}^{+}\right], 10 \%\right\}$.

\section{4-(n-Butylthio)- $N$-(4-methylphenyl)-1 $H$-pyrazole-3-carboxamide 75}

A solution of $N$-(4-methylphenyl)-Z-3-chloro-2-( $n$-butylthio)propenamide 30 (0.21 g, 0.7 mmol) in ether $(15 \mathrm{~mL})$ was added to an excess of an ethereal solution of diazomethane [prepared from Diazald $\left.{ }^{\circledR}(0.84 \mathrm{~g}, 3.9 \mathrm{mmol})\right]$ cooled in an ice-salt bath while stirring. The reaction solution was allowed to return slowly to room temperature while stirring for $4 \mathrm{~h}$. Following removal of the solvent and excess diazomethane, the crude product was obtained as a pale yellow oil. After purification by column chromatography on silica gel using hexane-ethyl acetate (gradient elution $20-80 \%$ ethyl acetate), 75 was obtained as a white solid (0.04 g, 20\%), mp 131-132 ${ }^{\circ} \mathrm{C} ; v_{\max } / \mathrm{cm}^{-1}(\mathrm{KBr}) 3286(\mathrm{NH}), 3110(\mathrm{NH}), 2923$ $(\mathrm{CH}), 1685(\mathrm{CO}), 1602,1541 ; \delta_{\mathrm{H}}\left(300 \mathrm{MHz}, \mathrm{CDCl}_{3}\right) 0.89$ [3H, t, J 7.2, C(4') $\left.H_{3}\right], 1.41$ [2H, sextet, $J$ 7.2, C(3') $\left.H_{2}\right], 1.52-1.67\left[2 \mathrm{H}, \mathrm{m}, \mathrm{C}\left(2^{\prime}\right) \mathrm{H}_{2}\right]^{*}, 2.34\left(3 \mathrm{H}, \mathrm{s}, \mathrm{ArCH}_{3}\right), 2.76(2 \mathrm{H}$, dd, J 7.5, 7.2, $\left.\mathrm{SCH}_{2}\right), 7.20(2 \mathrm{H}, \mathrm{d}, J$ 8.4, $\mathrm{ArH}), 7.57(2 \mathrm{H}, \mathrm{d}, J 8.1, \mathrm{ArH}), 7.70[1 \mathrm{H}, \mathrm{s}$, $\mathrm{C}(5) H], 9.90(1 \mathrm{H}$, br s, $\mathrm{N} H) ; \delta_{\mathrm{C}}\left(75.5 \mathrm{MHz}, \mathrm{CDCl}_{3}\right) 13.6\left[\mathrm{CH}_{3}, C\left(4^{\prime}\right) \mathrm{H}_{3}\right], 21.0\left(\mathrm{CH}_{3}\right.$, $\operatorname{ArCH} 3), 21.7\left[\mathrm{CH}_{2}, C\left(3^{\prime}\right) \mathrm{H}_{2}\right], 31.3\left[\mathrm{CH}_{2}, C\left(2^{\prime}\right) \mathrm{H}_{2}\right], 37.6\left(\mathrm{CH}_{2}, \mathrm{SCH}_{2}\right), 108.7[\mathrm{C}, C(4)]$, 120.2, $129.7(2 \times \mathrm{CH}$, aromatic $\mathrm{CH}), 134.4,135.2(2 \times \mathrm{C}$, aromatic $C), 140.5[\mathrm{C}$, br, $C(3)], 158.6(\mathrm{C}, C \mathrm{O})$; HRMS (ES+): Exact mass calculated for $\mathrm{C}_{15} \mathrm{H}_{20} \mathrm{~N}_{3} \mathrm{OS}[\mathrm{M}+\mathrm{H}]^{+}$, 
290.1327. Found 290.1319; m/z (ES+) $290.2\left\{\left[\left(\mathrm{C}_{15} \mathrm{H}_{19} \mathrm{~N}_{3} \mathrm{OS}\right)+\mathrm{H}^{+}\right], 100 \%\right\}, \quad 579.3$ $\left\{\left[\left(\mathrm{C}_{30} \mathrm{H}_{38} \mathrm{~N}_{6} \mathrm{O}_{2} \mathrm{~S}_{2}\right)+\mathrm{H}^{+}\right], 8 \%\right\}$.

*This signal sharpened in the ${ }^{1} \mathrm{H}$ NMR spectrum with a $\mathrm{D}_{2} \mathrm{O}$ shake to give a quintet at $1.60 \mathrm{ppm}(J 7.2)$.

\section{$N$-(4-Methylphenyl)-5-phenyl-1 $H$-pyrazole-3-carboxamide $79^{6}$}

a) Prepared from N-(4-methylphenyl)-Z-3-chloro-2-(benzenesulfinyl)propenamide $\mathbf{1 6}$

A solution of $N$-(4-methylphenyl)-Z-3-chloro-2-(benzenesulfinyl)propenamide 16 (0.20 $\mathrm{g}, 0.7 \mathrm{mmol})$ in ether $(10 \mathrm{~mL})$ and acetone $(2 \mathrm{~mL})$ was added dropwise to an excess of phenyldiazomethane [generated from benzaldehyde tosylhydrazone $(1.67 \mathrm{~g}, 6.0 \mathrm{mmol})$ ] cooled to $-50{ }^{\circ} \mathrm{C}$ with a cryocooler. The solution was allowed to return slowly to room temperature while stirring for $16 \mathrm{~h}$. After removal of the solvent by evaporation at reduced pressure, the crude product was obtained as an orange solid. Following purification by column chromatography on silica gel using hexane-ethyl acetate (gradient elution $10-40 \%$ ethyl acetate) as eluent, 79 was obtained as a pale yellow solid (0.10 g, 56\%), mp 228-230 ${ }^{\circ} \mathrm{C} ; v_{\max } / \mathrm{cm}^{-1}(\mathrm{KBr}) 3378(\mathrm{NH}), 3198(\mathrm{NH}), 2919(\mathrm{CH}), 1660(\mathrm{CO})$, $1596 ; \delta_{\mathrm{H}}\left(300 \mathrm{MHz}, \mathrm{DMSO}-d_{6}\right) 2.30\left(3 \mathrm{H}, \mathrm{s}, \mathrm{ArCH} \mathrm{H}_{3}\right), 7.01-8.01[10 \mathrm{H}, \mathrm{m}, \mathrm{ArH} \& \mathrm{C}(4) H$ (tentatively assigned as a br s at $7.28 \mathrm{ppm})], 10.07\left(1 \mathrm{H}\right.$, br s, $\mathrm{N} H$ of carboxamide); $\delta_{\mathrm{C}}$ (75.5 MHz, DMSO-d $\left.d_{6}\right) 20.5\left(\mathrm{CH}_{3}, \mathrm{ArCH}_{3}\right), 102.9[\mathrm{CH}, C(4) \mathrm{H}], 120.2,125.3,128.3$, $129.0(4 \times \mathrm{CH}, 4 \times$ aromatic $C \mathrm{H}), 132.5,136.1(2 \times \mathrm{C}, 2 \times$ aromatic $C), 159.2(\mathrm{C}, C \mathrm{O})$. There was a broad signal in the region 127-131 ppm and the signals for $\mathrm{C}(3), \mathrm{C}(5)$, one aromatic $\mathrm{C}$ and one aromatic $\mathrm{CH}$ were not detected in the ${ }^{13} \mathrm{C}$ NMR spectrum; HRMS (ES+): Exact mass calculated for $\mathrm{C}_{17} \mathrm{H}_{16} \mathrm{~N}_{3} \mathrm{O}[\mathrm{M}+\mathrm{H}]^{+}, 278.1293$. Found 278.1306; m/z (ES+) $278.0\left\{\left[\left(\mathrm{C}_{17} \mathrm{H}_{15} \mathrm{~N}_{3} \mathrm{O}\right)+\mathrm{H}^{+}\right], 100 \%\right\}$.

\section{b) Prepared from N-(4-methylphenyl)-Z-3-chloro-2-(n-butylsulfinyl)propenamide 31}

The title compound was also prepared by addition of a solution of $N$-(4-methylphenyl)-Z3-chloro-2-(n-butylsulfinyl)propenamide $31(0.15 \mathrm{~g}, 0.5 \mathrm{mmol})$ in ether $(10 \mathrm{~mL})$ to an excess of phenyldiazomethane [generated from benzaldehyde tosylhydrazone (1.55 g, 5.6 mmol)]. A precipitate formed as the reaction progressed. Following stirring at room temperature for $16 \mathrm{~h}$ the reaction was filtered through a sintered glass funnel (grade 4 ) to give 79 as a white solid (0.05 g, 38\%), mp 228-230 ${ }^{\circ} \mathrm{C}$; (Found $\mathrm{C}, 73.02 ; \mathrm{H}, 5.35$; N, 
15.17. $\mathrm{C}_{17} \mathrm{H}_{15} \mathrm{~N}_{3} \mathrm{O}$ requires $\left.\mathrm{C}, 73.63 ; \mathrm{H}, 5.45 ; \mathrm{N}, 15.15 \%\right) ; v_{\max } / \mathrm{cm}^{-1}(\mathrm{KBr}) 3379(\mathrm{NH})$, $3195(\mathrm{NH}), 3017(\mathrm{CH}), 2917(\mathrm{CH}), 1661(\mathrm{CO}), 1597$; $\delta_{\mathrm{H}}\left(300 \mathrm{MHz}, \mathrm{CDCl}_{3}\right) 2.35$ (3H, s, $\left.\mathrm{ArCH}_{3}\right), 7.16$ [1H, s, C(4)H], $7.18(2 \mathrm{H}, \mathrm{d}, J$ 8.4, $\mathrm{ArH}), 7.38-7.52(3 \mathrm{H}, \mathrm{m}, \mathrm{ArH}), 7.55-7.66$ $(4 \mathrm{H}, \mathrm{m}, \mathrm{Ar} H), 8.63\left(1 \mathrm{H}\right.$, br s, $\mathrm{N} H$ of carboxamide); $\delta_{\mathrm{H}}\left(300 \mathrm{MHz}, \mathrm{DMSO}-d_{6}\right) 2.29(3 \mathrm{H}, \mathrm{s}$, $\left.\mathrm{ArCH}_{3}\right), 7.17(2 \mathrm{H}, \mathrm{d}, J$ 8.4, $\mathrm{ArH}), 7.27$ [1H, br s, C(4)H], 7.37-7.55 (3H, m, ArH), 7.68 $(2 \mathrm{H}, \mathrm{d}, J 8.4, \mathrm{Ar} H), 7.84(2 \mathrm{H}, \mathrm{d}, J 7.2, \mathrm{Ar} H)$. There was an additional set of signals present at $\delta_{\mathrm{H}}\left(300 \mathrm{MHz}, \mathrm{DMSO}-d_{6}\right) 5.72(\mathrm{~s}), 8.27(\mathrm{~s}), 10.04\left(\right.$ br s); $\delta_{\mathrm{C}}(75.5 \mathrm{MHz}$, DMSO-d $\left.d_{6}\right) 20.5\left(\mathrm{CH}_{3}, \mathrm{ArCH} 3\right), 102.9[\mathrm{CH}, \mathrm{C}(4) \mathrm{H}], 120.2,125.3,128.3,128.97,128.99$ $(5 \times \mathrm{CH}, 5 \times$ aromatic $\mathrm{CH}), 132.6,136.1(2 \times \mathrm{C}, 2 \times$ aromatic $C), 159.2(\mathrm{C}, \mathrm{CO})$. The signals for $\mathrm{C}(3)$ and $\mathrm{C}(5)$ were not detected in the ${ }^{13} \mathrm{C}$ NMR spectrum.

HRMS (ES+): Exact mass calculated for $\mathrm{C}_{17} \mathrm{H}_{16} \mathrm{~N}_{3} \mathrm{O}[\mathrm{M}+\mathrm{H}]^{+}, 278.1293$. Found $278.1282 ; \mathrm{m} / \mathrm{z}(\mathrm{ES}+) 278.0\left\{\left[\left(\mathrm{C}_{17} \mathrm{H}_{15} \mathrm{~N}_{3} \mathrm{O}\right)+\mathrm{H}^{+}\right], 100 \%\right\}$.

\section{$\left(3 R^{*}, 4 R^{*}, 5 R^{*}, S_{\mathrm{S}}^{*}\right)-3-($ Benzylsulfinyl)-4-chloro-4,5-dihydro- $N$-methyl-5-phenyl-3H-}

\section{pyrazole-3-carboxamide $77 \& N$-methyl-5-phenyl-1 $H$-pyrazole-3-carboxamide 82}

The reaction was conducted by addition of a solution of $N$-methyl-Z-3-chloro-2(benzylsulfinyl)propenamide $9(0.18 \mathrm{~g}, 0.7 \mathrm{mmol})$ in ether $(10 \mathrm{~mL})$ to an excess of phenyldiazomethane [generated from benzaldehyde tosylhydrazone (1.40 g, $5.0 \mathrm{mmol})$ ] cooled to $-50{ }^{\circ} \mathrm{C}$ using a cryocooler. The solution was allowed to return slowly to room temperature while stirring for $16 \mathrm{~h}$. A precipitate formed as the reaction progressed. The product was collected by filtration through a sintered glass funnel (grade 3) to give $\mathbf{7 7}$ as a white solid $(0.10 \mathrm{~g}, 37 \%)$ as a single diastereomer, mp $76-78{ }^{\circ} \mathrm{C}$; (Found $\mathrm{C}, 55.90 ; \mathrm{H}$, $4.91 ; \mathrm{N}, 10.58 . \mathrm{C}_{18} \mathrm{H}_{18} \mathrm{ClN}_{3} \mathrm{O}_{2} \mathrm{~S}$ requires $\left.\mathrm{C}, 57.52 ; \mathrm{H}, 4.83 ; \mathrm{N}, 11.18 \%\right) ; v_{\max } / \mathrm{cm}^{-1}(\mathrm{KBr})$ $3320(\mathrm{NH}), 3034(\mathrm{CH}), 2931(\mathrm{CH}), 1671(\mathrm{CO}), 1532(\mathrm{~N}=\mathrm{N}), 1047(\mathrm{SO}) ; \delta_{\mathrm{H}}(300 \mathrm{MHz}$, $\left.\mathrm{CDCl}_{3}\right) 3.02\left(3 \mathrm{H}, \mathrm{d}, J 4.8, \mathrm{NHCH}_{3}\right), 4.21\left(1 \mathrm{H}, \mathrm{d}, \mathrm{A}\right.$ of AB system, $J 12.8$, one of $\left.\mathrm{SCH}_{2}\right)$, $4.40\left(1 \mathrm{H}, \mathrm{d}, \mathrm{B}\right.$ of AB system, $J$ 12.8, one of $\left.\mathrm{SCH}_{2}\right), 4.69$ [1H, d, $\left.J 6.8, \mathrm{C}(4) H \mathrm{Cl}\right], 5.94$ $\left[1 \mathrm{H}, \mathrm{d}, J\right.$ 6.4, C(5)HPh], $7.00(1 \mathrm{H}$, br t, $\mathrm{NH}), 7.20-7.50(14 \mathrm{H}, \mathrm{m}, \mathrm{ArH}) ; \delta_{\mathrm{C}}(75.5 \mathrm{MHz}$, $\left.\mathrm{CDCl}_{3}\right) 26.8\left(\mathrm{CH}_{3}, \mathrm{NHCH}_{3}\right), 56.3\left(\mathrm{CH}_{2}, \mathrm{SCH}_{2}\right), 59.3[\mathrm{CH}, \mathrm{C}(4) \mathrm{HCl}], 99.8[\mathrm{CH}$, $C(5) \mathrm{HPh}], 107.5$ [C, $C(3)], 127.0,128.9,129.1,129.2,129.3(5 \times \mathrm{CH}, 5 \times$ aromatic $C \mathrm{H})$, $129.9(\mathrm{C}$, aromatic $C), 130.5(\mathrm{CH}$, aromatic $C \mathrm{H}), 134.2(\mathrm{C}$, aromatic $C), 162.8(\mathrm{C}, \mathrm{CO})$; HRMS (ES+): Exact mass calculated for $\mathrm{C}_{18} \mathrm{H}_{19} \mathrm{NO}_{2} \mathrm{~S}^{35} \mathrm{Cl}\left[(\mathrm{M}+\mathrm{H})^{+}-\mathrm{N}_{2}\right], 348.0825$. 
Found $\quad 348.0816 ; \quad \mathrm{m} / \mathrm{z} \quad(\mathrm{ES}+) \quad 350.0 \quad\left\{\left[\left(\mathrm{C}_{18} \mathrm{H}_{18} \mathrm{NO}_{2} \mathrm{~S}^{37} \mathrm{Cl}\right)+\mathrm{H}^{+}\right], \quad 8 \%\right\}, \quad 348.2$ $\left\{\left[\left(\mathrm{C}_{18} \mathrm{H}_{18} \mathrm{NO}_{2} \mathrm{~S}^{35} \mathrm{Cl}\right)+\mathrm{H}^{+}\right], 20 \%\right\}, 202.2\left\{\left[\left(\mathrm{C}_{11} \mathrm{H}_{11} \mathrm{~N}_{3} \mathrm{O}\right)+\mathrm{H}^{+}\right], 8 \%\right\}, 87.9(100 \%)$.

$\left(3 R^{*}, 4 R^{*}, 5 R^{*}, S_{\mathrm{S}} *\right)-N$-Benzyl-3-(benzylsulfinyl)-4-chloro-4,5-dihydro-5-phenyl-3Hpyrazole-3-carboxamide $78 \& N$-benzyl-5-phenyl-1 $H$-pyrazole-3-carboxamide 80

A solution of $N$-benzyl-Z-3-chloro-2-(benzylsulfinyl)propenamide 10 (0.21 g, $0.6 \mathrm{mmol})$ in ether $(12 \mathrm{~mL})$ was added dropwise to an excess of phenyldiazomethane [generated from benzaldehyde tosylhydrazone $(1.61 \mathrm{~g}, 5.9 \mathrm{mmol})]$ cooled to $-50{ }^{\circ} \mathrm{C}$ using a cryocooler. The solution was slowly allowed to return to room temperature and the reaction mixture was then stirred for $16 \mathrm{~h}$. A precipitate formed as the reaction progressed. The product was collected by filtration through a sintered glass funnel (grade $3)$ to give 78 as a white solid $(0.18 \mathrm{~g}, 64 \%)$ as a single diastereomer, mp $99-100{ }^{\circ} \mathrm{C}$; (Found C, 63.14; H, 4.76; N, 9.30. $\mathrm{C}_{24} \mathrm{H}_{22} \mathrm{ClN}_{3} \mathrm{O}_{2} \mathrm{~S}$ requires $\mathrm{C}, 63.78 ; \mathrm{H}, 4.91 ; \mathrm{N}, 9.30 \%$ ); $v_{\max } / \mathrm{cm}^{-1}(\mathrm{KBr}) 3310(\mathrm{NH}), 3029(\mathrm{CH}), 2983(\mathrm{CH}), 1678(\mathrm{CO}), 1525(\mathrm{~N}=\mathrm{N}), 1049(\mathrm{SO})$; $\delta_{\mathrm{H}}\left(300 \mathrm{MHz}, \mathrm{DMSO}-d_{6}\right) 4.17\left(1 \mathrm{H}, \mathrm{d}, \mathrm{A}\right.$ of $\mathrm{AB}$ system, $J_{\mathrm{AB}} 12.6$, one of $\left.\mathrm{SCH}_{2}\right), 4.27$ $\left(1 \mathrm{H}, \mathrm{d}, \mathrm{A}\right.$ of $\mathrm{AB}$ system, $J_{\mathrm{AB}} 12.6$, one of $\left.\mathrm{SCH}_{2}\right), 4.31\left(1 \mathrm{H}, \mathrm{dd}, \mathrm{A}\right.$ of $\mathrm{ABX}, J_{\mathrm{AB}} 14.4, J_{\mathrm{AX}}$ 5.7, one of $\left.\mathrm{NHCH}_{2}\right), 4.59\left(1 \mathrm{H}, \mathrm{dd}, \mathrm{A}\right.$ of $\mathrm{ABX}, J_{\mathrm{AB}} 14.4, J_{\mathrm{BX}} 6.6$, one of $\left.\mathrm{NHCH}_{2}\right), 4.86$ [1H, d, $J$ 8.7, C(4)HCl], 5.59 [1H, d, $J$ 8.7, C(5)HPh], 7.11-7.56 (15H, m, ArH), 9.44 $(1 \mathrm{H}$, br t $, J 6.3, \mathrm{~N} H)$. There was also evidence for decomposition to the pyrazole $\mathbf{8 0}$, with characteristic peaks at $\delta_{\mathrm{H}}\left(300 \mathrm{MHz}, \mathrm{DMSO}-d_{6}\right) 4.48\left(2 \mathrm{H}, \mathrm{d}, J 6.0, \mathrm{NHCH}_{2}\right), 7.18[1 \mathrm{H}, \mathrm{s}$, $\mathrm{C}(4) H], 7.81(2 \mathrm{H}, \mathrm{d}, J 8.4, \mathrm{Ar} H), 8.89(1 \mathrm{H}$, br t, $J 6.0, \mathrm{~N} H$ of carboxamide), a broad signal for water was also observed at $3.61 \mathrm{ppm}$ (water signal normally observed at 3.40 ppm). When the ${ }^{1} \mathrm{H}$ NMR spectrum was re-run after 20 hours, complete decomposition to the pyrazole $\mathbf{8 0}$ had occurred, with spectroscopic details consistent with a genuine sample of 80.

HRMS (ES+): Exact mass calculated for $\mathrm{C}_{24} \mathrm{H}_{23} \mathrm{NO}_{2} \mathrm{~S}^{35} \mathrm{Cl}\left[(\mathrm{M}+\mathrm{H})^{+}-\mathrm{N}_{2}\right], 424.1138$. Found 424.1136; m/z $(\mathrm{ES}+) 424.2\left\{\left[\left(\mathrm{C}_{24} \mathrm{H}_{22} \mathrm{NO}_{2} \mathrm{~S}^{35} \mathrm{Cl}\right)+\mathrm{H}^{+}\right], \quad 4 \%\right\}, \quad 278.2$ $\left\{\left[\left(\mathrm{C}_{17} \mathrm{H}_{15} \mathrm{~N}_{3} \mathrm{O}\right)+\mathrm{H}^{+}\right], 16 \%\right\}, 87.9(100 \%)$.

\section{$N$-Benzyl-5-phenyl-1 $H$-pyrazole-3-carboxamide 80}


a) Prepared from N-benzyl-Z-3-chloro-2-(benzenesulfinyl)propenamide 14 ('H NMR spectrum recorded in DMSO-d ${ }_{6}$ )

A solution of $N$-benzyl-Z-3-chloro-2-(benzenesulfinyl)propenamide $14(0.19 \mathrm{~g}, 0.6$ $\mathrm{mmol})$ in ether $(10 \mathrm{~mL})$ and acetone $(1 \mathrm{~mL})$ was added dropwise to an excess of phenyldiazomethane [generated from benzaldehyde tosylhydrazone $(1.67 \mathrm{~g}, 6.0 \mathrm{mmol})]$ cooled to $-50{ }^{\circ} \mathrm{C}$ with a cryocooler. The solution was allowed to return slowly to room temperature while stirring for $16 \mathrm{~h}$. After removal of the solvent by evaporation at reduced pressure, the crude product was obtained as a red oil. Following purification by column chromatography on silica gel using hexane: ethyl acetate (gradient elution 10$40 \%$ ethyl acetate), 80 was isolated as a white solid (0.13 g, 79\%), mp 178-180 ${ }^{\circ} \mathrm{C}$; $v_{\max } / \mathrm{cm}^{-1}(\mathrm{KBr}) 3410(\mathrm{NH}), 3127(\mathrm{NH}), 3064(\mathrm{CH}), 2924(\mathrm{CH}), 1645(\mathrm{CO}), 1553 ; \delta_{\mathrm{H}}$ $\left(300 \mathrm{MHz}, \mathrm{DMSO}-d_{6}\right) 4.49\left(2 \mathrm{H}, \mathrm{d}, J 6.3, \mathrm{NHCH}_{2}\right), 7.18[1 \mathrm{H}, \mathrm{s}, \mathrm{C}(4) H], 7.22-7.55(8 \mathrm{H}$, m, $\operatorname{Ar} H), 7.81(2 \mathrm{H}, \mathrm{d}, J 7.8, \operatorname{Ar} H), 8.89\left(1 \mathrm{H}\right.$, br s, $\mathrm{NH}$ of carboxamide); $\delta_{\mathrm{C}}(75.5 \mathrm{MHz}$, DMSO- $\left.d_{6}\right) 42.0\left(\mathrm{CH}_{2}, \mathrm{NHCH}_{2}\right), 102.4[\mathrm{CH}, C(4) \mathrm{H}], 125.2,126.7,127.3,128.15,128.22$, $128.9(6 \times \mathrm{CH}, 6 \times$ aromatic $\mathrm{CH}), 139.6(\mathrm{C}$, aromatic $C), 160.6(\mathrm{C}, \mathrm{br}, \mathrm{CO})$. The signals for $\mathrm{C}(3), \mathrm{C}(5)$ and one aromatic $\mathrm{C}$ were not detected in the ${ }^{13} \mathrm{C}$ NMR spectrum.

HRMS (ES+): Exact mass calculated for $\mathrm{C}_{17} \mathrm{H}_{16} \mathrm{~N}_{3} \mathrm{O}[\mathrm{M}+\mathrm{H}]^{+}, 278.1293$. Found $278.1297 ; \mathrm{m} / \mathrm{z}(\mathrm{ES}+) 278.3\left\{\left[\left(\mathrm{C}_{17} \mathrm{H}_{15} \mathrm{~N}_{3} \mathrm{O}\right)+\mathrm{H}^{+}\right], 100 \%\right\}$.

b) Prepared from N-benzyl-Z-3-chloro-2-(n-butylsulfinyl)propenamide $27{ }^{1} \mathrm{H} N M R$ spectrum recorded in $\mathrm{CDCl}_{3}$ and $\mathrm{DMSO}-d_{6}$ )

The title compound was also prepared by addition of a solution of $N$-benzyl-Z-3-chloro2-(n-butylsulfinyl)propenamide $27(0.10 \mathrm{~g}, 0.3 \mathrm{mmol})$ in ether $(10 \mathrm{~mL})$ to an excess of phenyldiazomethane [generated from benzaldehyde tosylhydrazone (1.56 g, $5.6 \mathrm{mmol})$ ]. A precipitate formed as the reaction progressed. Following stirring at room temperature for $16 \mathrm{~h}$ the reaction was filtered through a sintered glass funnel (grade 4 ) to give $\mathbf{8 0}$ as a white solid (0.03 g, 36\%), mp 177-178 ${ }^{\circ} \mathrm{C} ; v_{\max } / \mathrm{cm}^{-1}(\mathrm{KBr}) 3410(\mathrm{NH}), 3128(\mathrm{NH}), 3066$ $(\mathrm{CH}), 2924(\mathrm{CH}), 1643(\mathrm{CO}), 1554 ; \delta_{\mathrm{H}}\left(300 \mathrm{MHz}, \mathrm{CDCl}_{3}\right) 4.66\left(2 \mathrm{H}, \mathrm{d}, J\right.$ 6.0, $\left.\mathrm{NHCH}_{2}\right)$, $7.08[1 \mathrm{H}, \mathrm{s}, \mathrm{C}(4) H], 7.13(1 \mathrm{H}, \mathrm{br} \mathrm{s}, \mathrm{N} H$ of carboxamide $), 7.22-7.51(8 \mathrm{H}, \mathrm{m}, \mathrm{Ar} H), 7.60$ $(2 \mathrm{H}, \mathrm{d}, J 7.5, \operatorname{Ar} H) ; \delta_{\mathrm{H}}\left(300 \mathrm{MHz}, \mathrm{DMSO}-d_{6}\right) 4.48\left(2 \mathrm{H}, \mathrm{d}, J 6.0, \mathrm{NHCH}_{2}\right), 7.09$ [1H, br s, $\mathrm{C}(4) H], 7.18-7.56(8 \mathrm{H}, \mathrm{m}, \operatorname{Ar} H), 7.80(2 \mathrm{H}, \mathrm{d}, J 7.5, \operatorname{Ar} H), 8.75(1 \mathrm{H}, \mathrm{br}, \mathrm{N} H$ of 
carboxamide). HRMS (ES+): Exact mass calculated for $\mathrm{C}_{17} \mathrm{H}_{16} \mathrm{~N}_{3} \mathrm{O}[\mathrm{M}+\mathrm{H}]^{+}, 278.1293$. Found 278.1307; m/z (ES+) $278.3\left\{\left[\left(\mathrm{C}_{17} \mathrm{H}_{15} \mathrm{~N}_{3} \mathrm{O}\right)+\mathrm{H}^{+}\right], 100 \%\right\}$.

$N$-Methyl-5-phenyl-1 $H$-pyrazole-3-carboxamide $82 \&\left(3 R^{*}, 4 R^{*}, 5 R^{*}, S_{\mathrm{S}}^{*}\right)$-4-Chloro4,5-dihydro- $N$-methyl-5-phenyl-3-(benzenesulfinyl)-3H-pyrazole-3-carboxamide 81

A solution of $N$-methyl-Z-3-chloro-2-(benzenesulfinyl)propenamide 15 (0.21 g, 0.9 $\mathrm{mmol})$ in ether $(10 \mathrm{~mL})$ was added dropwise to an excess of phenyldiazomethane [generated from benzaldehyde tosylhydrazone $(1.67 \mathrm{~g}, 6.0 \mathrm{mmol})$ ] cooled to $-50{ }^{\circ} \mathrm{C}$ with a cryocooler. The solution was allowed to return slowly to room temperature and the reaction mixture was then stirred for $16 \mathrm{~h}$. A precipitate formed as the reaction progressed. The crude product was collected by filtration through a sintered glass funnel (grade 3) to give a mixture of $\mathbf{8 2}$ and $\mathbf{8 1}$ (82:81 9:1 by ${ }^{1} \mathrm{H}$ NMR spectroscopic analysis) as white solid $(0.18 \mathrm{~g}, 64 \%)$. Following trituration in ether, $\mathbf{8 2}$ and $\mathbf{8 1}$ were obtained as a pale yellow solid (0.03 g, 17\%) (82:81 9:1 by ${ }^{1} \mathrm{H}$ NMR spectroscopy), mp 161-162 ${ }^{\circ} \mathrm{C}$; $v_{\max } / \mathrm{cm}^{-1}(\mathrm{KBr}) 3401(\mathrm{NH}), 3272(\mathrm{NH}), 3044(\mathrm{CH}), 1669(\mathrm{CO}), 1571$;

82: $\delta_{\mathrm{H}}\left(300 \mathrm{MHz}, \mathrm{CDCl}_{3}\right) 3.00\left(3 \mathrm{H}, \mathrm{d}, J 4.8, \mathrm{NHCH}_{3}\right), 6.99(1 \mathrm{H}, \mathrm{br}, \mathrm{N} H$ of carboxamide), $7.07[1 \mathrm{H}, \mathrm{s}, \mathrm{C}(4) H], 7.33-7.51(3 \mathrm{H}, \mathrm{m}, \operatorname{Ar} H), 7.58-7.70(2 \mathrm{H}, \mathrm{m}, \operatorname{Ar} H), \mathrm{a}$ broad water signal was observed at $2.88 \mathrm{ppm}$ (water signal is normally seen at $1.60 \mathrm{ppm}$ ); $\delta_{\mathrm{H}}\left(300 \mathrm{MHz}, \mathrm{DMSO}-d_{6}\right) 2.78\left(3 \mathrm{H}\right.$, br s, $\left.\mathrm{NHCH}_{3}\right), 7.01-7.54[4 \mathrm{H}, \mathrm{m}, \mathrm{C}(4) H \& \mathrm{Ar} H]$, 7.75-7.84 (2H, br m, $\operatorname{Ar} H), 8.33(1 \mathrm{H}$, br s, $\mathrm{N} H$ of carboxamide). $\mathrm{N}(1) H$ of pyrazole was unresolved in both $\mathrm{CDCl}_{3}$ and DMSO- $d_{6} ; \delta_{\mathrm{C}}\left(75.5 \mathrm{MHz}, \mathrm{DMSO}-d_{6}\right) 25.5\left(\mathrm{CH}_{3}, \mathrm{NHCH}_{3}\right)$, 102.2 [CH, $C(4) \mathrm{H}], 125.1,128.1,128.9(3 \times \mathrm{CH}, 3 \times$ aromatic $\mathrm{CH}), 130.3(\mathrm{C}$, aromatic C), 144.6, $145.8[2 \times \mathrm{C}, C(3) \& C(5)], 161.1(\mathrm{C}, C \mathrm{O})$; HRMS (ES+): Exact mass calculated for $\mathrm{C}_{11} \mathrm{H}_{12} \mathrm{~N}_{3} \mathrm{O}[\mathrm{M}+\mathrm{H}]^{+}, 202.0980$. Found 202.0984; m/z (ES+) 202.0 $\left\{\left[\left(\mathrm{C}_{11} \mathrm{H}_{11} \mathrm{~N}_{3} \mathrm{O}\right)+\mathrm{H}^{+}\right], 100 \%\right\}$.

Characteristic peaks for 81 were seen at $\delta_{\mathrm{H}}\left(300 \mathrm{MHz}, \mathrm{CDCl}_{3}\right) 4.68[1 \mathrm{H}, \mathrm{d}, J 10.8$, $\mathrm{C}(4) H \mathrm{Cl}], 5.53[1 \mathrm{H}, \mathrm{d}, J 10.5, \mathrm{C}(5) H \mathrm{Ph}], 9.24(1 \mathrm{H}, \mathrm{br} \mathrm{s}, \mathrm{NH})$ and at $\delta_{\mathrm{H}}(300 \mathrm{MHz}$, DMSO-d $\left.d_{6}\right) 5.04$ [1H, br s, C(4)HCl], $5.55[1 \mathrm{H}, \mathrm{d}, J 10.0, \mathrm{C}(5) H \mathrm{Ph}], 8.98(1 \mathrm{H}$, br s, $\mathrm{N} H)$.

\section{$N$-Benzyl-4-(benzylthio)-5-phenyl-1 $H$-pyrazole-3-carboxamide 83}


A solution of $N$-benzyl-Z-3-chloro-2-(benzylthio)propenamide $28(0.20 \mathrm{~g}, 0.6 \mathrm{mmol})$ in ether $(10 \mathrm{~mL})$ was added dropwise to an excess of phenyldiazomethane [generated from benzaldehyde tosylhydrazone $(1.51 \mathrm{~g}, 5.4 \mathrm{mmol})]$ cooled to $-50{ }^{\circ} \mathrm{C}$ with a cryocooler. The solution was allowed to return slowly to room temperature while stirring for $16 \mathrm{~h}$. Following removal of the solvent and excess phenyldiazomethane by evaporation under reduced pressure, the crude product was obtained as a red oil. The ${ }^{1} \mathrm{H}$ NMR spectrum of the crude product was very complex, with evidence for the pyrazoline at $\delta_{\mathrm{H}} 5.60[\mathrm{~d}, J 8.4$, $\mathrm{C}(5) H \mathrm{Ph}$ ]. Purification by column chromatography using hexane-ethyl acetate (gradient elution $10-20 \%$ ethyl acetate) gave 83 as a white solid (0.05 g, 19\%), mp 157-158 ${ }^{\circ} \mathrm{C}$; $v_{\max } / \mathrm{cm}^{-1}(\mathrm{KBr}) 3264(\mathrm{NH}), 3181(\mathrm{NH}), 2924(\mathrm{CH}), 1645(\mathrm{CO}), 1602,1559 ; \delta_{\mathrm{H}}(300$ $\left.\mathrm{MHz} \mathrm{CDCl}_{3}\right) 3.65(2 \mathrm{H}, \mathrm{s}, \mathrm{SCH}), 4.48\left(2 \mathrm{H}, \mathrm{d}, J\right.$ 5.8, $\left.\mathrm{NHCH}_{2}\right), 6.73-6.82(2 \mathrm{H}, \mathrm{m}, \mathrm{ArH})$, 7.04-7.50 (11H, m, ArH), $7.93(2 \mathrm{H}, \mathrm{dd}, J$ 8.1, 1.8, $\mathrm{Ar} H), 8.19(1 \mathrm{H}$, br t, $\mathrm{N} H$ of carboxamide); $\delta_{\mathrm{C}}\left(75.5 \mathrm{MHz}, \mathrm{CDCl}_{3}\right)$ 41.0, $43.4\left(2 \times \mathrm{CH}_{2}, \mathrm{SCH}_{2} \& \mathrm{NHCH}_{2}\right), 105.3[\mathrm{C}$, $C(4)], 127.5,127.7,127.85,127.88,128.2,128.5,128.6,128.8,129.1(9 \times \mathrm{CH}, 9 \times$ aromatic $\mathrm{CH}), 131.8,136.5,137.5(3 \times \mathrm{C}, 3 \times$ aromatic $C), 158.9(\mathrm{C}, \mathrm{CO})$, the signals for $\mathrm{C}(3)$ and $\mathrm{C}(5)$ were not detected in the ${ }^{13} \mathrm{C}$ NMR spectrum; HRMS (ES+): Exact mass calculated for $\mathrm{C}_{24} \mathrm{H}_{22} \mathrm{~N}_{3} \mathrm{OS}[\mathrm{M}+\mathrm{H}]^{+}$, 400.1484. Found 400.1497; m/z (ES+) 400.0 $\left\{\left[\left(\mathrm{C}_{24} \mathrm{H}_{21} \mathrm{~N}_{3} \mathrm{OS}\right)+\mathrm{H}^{+}\right], 100 \%\right\}$.

\section{4-(Benzylthio)-5-phenyl- $N$-(4-methylphenyl)-1 $H$-pyrazole-3-carboxamide 84}

A solution of $N$-(4-methylphenyl)-Z-3-chloro-2-(benzylthio)propenamide 20 (0.17 g, 0.5 $\mathrm{mmol})$ in ether $(10 \mathrm{~mL})$ was added dropwise to an excess of phenyldiazomethane [generated from benzaldehyde tosylhydrazone $(1.54 \mathrm{~g}, 5.5 \mathrm{mmol})$ ] cooled to $-50{ }^{\circ} \mathrm{C}$ with a cryocooler. The solution was allowed to return slowly to room temperature while stirring for $16 \mathrm{~h}$. Following removal of the solvent and excess phenyldiazomethane by evaporation under reduced pressure, the crude product was obtained as a red oil. In the ${ }^{1} \mathrm{H}$ NMR spectrum of the crude product, there was evidence for the pyrazoline at $\delta_{\mathrm{H}} 4.49$ [d, $J$ 8.4, C(4)HCl], 5.63 [d, $J$ 8.4, C(5)HPh]. Purification by column chromatography using hexane-ethyl acetate (gradient elution 10-15\% ethyl acetate) gave 84 as a white solid (0.05 g, 24\%), mp 191-192 ${ }^{\circ} \mathrm{C}$; (Found C, 71.98; H, 5.58; N, 10.12; S, 8.09. $\mathrm{C}_{24} \mathrm{H}_{21} \mathrm{~N}_{3} \mathrm{OS}$ requires $\mathrm{C}, 72.15 ; \mathrm{H}, 5.30 ; \mathrm{N}, 10.52 ; \mathrm{S}, 8.03 \%) ; v_{\max } / \mathrm{cm}^{-1}(\mathrm{KBr}) 3398(\mathrm{NH}), 3190(\mathrm{NH})$, $2921(\mathrm{CH}), 1660(\mathrm{CO}), 1604,1525 ; \delta_{\mathrm{H}}\left(300 \mathrm{MHz}, \mathrm{CDCl}_{3}\right) 2.35\left(3 \mathrm{H}, \mathrm{s}, \mathrm{ArCH}_{3}\right), 3.78$ 
(2H, s, SCH$), 6.85-6.96(2 \mathrm{H}, \mathrm{m}, \mathrm{ArH}), 7.03-7.12(3 \mathrm{H}, \mathrm{m}, \mathrm{ArH}), 7.16(2 \mathrm{H}, \mathrm{d}, J$ 8.1, $\mathrm{ArH})$, 7.38-7.52 (5H, m, ArH), 7.95 (2H, br d, J 6.3, $\mathrm{Ar} H), 9.70$ (1H, br s, NH of carboxamide), $11.65[1 \mathrm{H}, \mathrm{v}$ br s, $\mathrm{N}(1) H]$; $\delta_{\mathrm{C}}\left(75.5 \mathrm{MHz}, \mathrm{DMSO}-d_{6}\right) 20.4\left(\mathrm{CH}_{3}, \mathrm{ArCH}\right), 40.0\left(\mathrm{CH}_{2}\right.$, $\left.\mathrm{SCH}_{2}\right), 106.5$ [C, br, $\left.C(4)\right], 120.0,126.8,128.0,128.1,128.3,128.7,129.1(7 \times \mathrm{CH}, 7 \times$ aromatic $\mathrm{CH}, 7$ signals for 8 carbons $), 132.7,136.0,137.6(3 \times \mathrm{C}, 3 \times$ aromatic $C), 146.4$, 149.5 [2 $\times \mathrm{C}, C(3)$ and $C(5)], 160.4(\mathrm{C}, C \mathrm{O})$; HRMS (ES+): Exact mass calculated for $\mathrm{C}_{24} \mathrm{H}_{22} \mathrm{~N}_{3} \mathrm{OS} \quad[\mathrm{M}+\mathrm{H}]^{+}, \quad 400.1484$. Found 400.1476; m/z $\quad$ (ES+) 400.0 $\left\{\left[\left(\mathrm{C}_{24} \mathrm{H}_{21} \mathrm{~N}_{3} \mathrm{OS}\right)+\mathrm{H}^{+}\right], 100 \%\right\}$.

$\left(3 R^{*}, 4 R^{*}, 5 R^{*}\right)-4-C h l o r o-4,5-d i h y d r o-N$-methyl-5-phenyl-3-(phenylthio)-3Hpyrazole-3-carboxamide $86, \mathrm{~N}$-methyl-5-phenyl-1 $\mathrm{H}$-pyrazole-3-carboxamide $82^{47} \&$ $\mathrm{N}$-methyl-5-phenyl-4-(phenylthio)-1 $\mathrm{H}$-pyrazole-3-carboxamide 90

A solution of $N$-methyl-Z-3-chloro-2-(phenylthio)propenamide $25(0.15 \mathrm{~g}, 0.7 \mathrm{mmol})$ in ether $(10 \mathrm{~mL})$ was added dropwise to an excess of phenyldiazomethane [generated from benzaldehyde tosylhydrazone $(1.57 \mathrm{~g}, 5.6 \mathrm{mmol})]$ cooled to $-50{ }^{\circ} \mathrm{C}$ with a cryocooler. The solution was allowed to return slowly to room temperature while stirring for $16 \mathrm{~h}$. A precipitate formed as the reaction progressed. The product was collected by filtration through a sintered glass funnel (grade 3) to give 86 as a white solid $(0.06 \mathrm{~g}, 25 \%)$ as a single diastereomer, mp 93-94 ${ }^{\circ} \mathrm{C} ; v_{\max } / \mathrm{cm}^{-1}(\mathrm{KBr}) 3399(\mathrm{NH}), 2945(\mathrm{CH}), 1667$ (CO), $1528(\mathrm{~N}=\mathrm{N}) ; \delta_{\mathrm{H}}\left(300 \mathrm{MHz}, \mathrm{CDCl}_{3}\right) 2.65\left(3 \mathrm{H}, \mathrm{d}, J 5.0, \mathrm{NHCH}_{3}\right), 4.48[1 \mathrm{H}, \mathrm{d}, J$ 7.6, $\mathrm{C}(4) H \mathrm{Cl}], 5.40[1 \mathrm{H}, \mathrm{d}, J$ 7.6, C(5)HPh], $6.28(1 \mathrm{H}, \mathrm{br} \mathrm{s}, \mathrm{NH}), 7.21-7.29(2 \mathrm{H}, \mathrm{m}, \operatorname{Ar} H)$, 7.35-7.51 (6H, m, ArH), 7.66-7.73 (2H, m, ArH); $\delta_{\mathrm{H}}\left(400 \mathrm{MHz}, \mathrm{DMSO}-\mathrm{d}_{6}\right) 2.49(3 \mathrm{H}, \mathrm{d}$, $J$ 4.4, $\left.\mathrm{NHCH}_{3}\right), 4.62,5.59[2 \times 1 \mathrm{H}, 2 \times \mathrm{d}, J 7.6, \mathrm{C}(5) H \mathrm{Ph} \& \mathrm{C}(4) H \mathrm{Cl}], 7.27-7.32(2 \mathrm{H}, \mathrm{m}$, $\operatorname{Ar} H), 7.40-7.55(6 \mathrm{H}, \mathrm{m}, \operatorname{Ar} H), 7.61-7.68(2 \mathrm{H}, \mathrm{m}, \operatorname{Ar} H), 8.08(1 \mathrm{H}, \mathrm{br} \mathrm{q}, J 4.4, \mathrm{~N} H)$; HRMS (ES+): Exact mass calculated for $\mathrm{C}_{17} \mathrm{H}_{17} \mathrm{NOS}^{35} \mathrm{Cl}\left[(\mathrm{M}+\mathrm{H})^{+}-\mathrm{N}_{2}\right], 318.0719$. Found $318.0730 ; \mathrm{m} / \mathrm{z}(\mathrm{ES}+) 320.0\left\{\left[\left(\mathrm{C}_{17} \mathrm{H}_{16} \mathrm{NOS}^{37} \mathrm{Cl}\right)+\mathrm{H}^{+}\right], \quad 16 \%\right\}, \quad 318.0$ $\left\{\left[\left(\mathrm{C}_{17} \mathrm{H}_{16} \mathrm{NOS}^{35} \mathrm{Cl}\right)+\mathrm{H}^{+}\right], 34 \%\right\}, 87.8(100 \%)$.

This compound decomposed in DMSO- $d_{6}$ to the rearranged pyrazole 90 and the pyrazole 82 (ratio of 90:82 1.5:1) with characteristic peaks for 90 seen at $\delta_{\mathrm{H}}\left(300 \mathrm{MHz}, \mathrm{DMSO}-d_{6}\right)$ $2.79\left(3 \mathrm{H}, \mathrm{d}, J 4.4, \mathrm{NHCH}_{3}\right), 8.30(1 \mathrm{H}, \mathrm{br} \mathrm{q}, J 4.4, \mathrm{~N} H$ of carboxamide $)$ and at $\delta_{\mathrm{C}}(75.5$ MHz, DMSO- $\left.d_{6}\right) 25.5\left(\mathrm{CH}_{3}, \mathrm{NHCH}_{3}\right), 102.1[\mathrm{C}, \mathrm{C}(4)], 125.1,128.1,128.9(3 \times \mathrm{CH}, 3 \times$ 
aromatic $C \mathrm{H}), 130.3(\mathrm{C}$, aromatic $C), 144.6,145.8[2 \times \mathrm{C}, C(3) \& C(5)], 161.1(\mathrm{C}, C \mathrm{O})$; and characteristic peaks for 82 seen at $\delta_{\mathrm{H}}\left(300 \mathrm{MHz}, \mathrm{DMSO}-d_{6}\right) 2.74(3 \mathrm{H}, \mathrm{d}, J 4.4$, $\left.\mathrm{NHCH}_{3}\right), 8.23\left(1 \mathrm{H}\right.$, br q, $J 4.8, \mathrm{~N} H$ of carboxamide) and at $\delta_{\mathrm{C}}\left(75.5 \mathrm{MHz}, \mathrm{DMSO}-d_{6}\right)$ $27.7\left(\mathrm{CH}_{3}, \mathrm{NHCH}_{3}\right), 102.2[\mathrm{CH}, \mathrm{C}(4) \mathrm{H}], 124.1,125.3(2 \times \mathrm{CH}$, aromatic $\mathrm{CH}), 125.0(\mathrm{C}$, aromatic $C), 127.6,129.0,129.3,129.6(4 \times \mathrm{CH}$, aromatic $C H), 130.5,138.1,143.6[3 \times$ $\mathrm{C}$, aromatic $C, C(3)$ or $C(5)], 160.9(\mathrm{C}, C \mathrm{O})$. Spectroscopic details agreed with a genuine sample of 82 .

\section{4-(Phenylthio)-5-phenyl- $N$-(4-methylphenyl)- $1 H$-pyrazole-3-carboxamide 85}

A solution of $N$-(4-methylphenyl)-Z-3-chloro-2-(phenylthio)propenamide 24 (0.20 g, 0.7 $\mathrm{mmol})$ in ether $(10 \mathrm{~mL})$ was added dropwise to an excess of phenyldiazomethane [generated from benzaldehyde tosylhydrazone $(1.55 \mathrm{~g}, 5.5 \mathrm{mmol})$ ] cooled to $-50{ }^{\circ} \mathrm{C}$ with a cryocooler. The solution was allowed to return slowly to room temperature while stirring for $16 \mathrm{~h}$. Following removal of the solvent and excess phenyldiazomethane by evaporation under reduced pressure, the crude product was obtained as an orange oil. The ${ }^{1} \mathrm{H}$ NMR spectrum of the crude product was very complex, with evidence for the pyrazoline at $\delta_{\mathrm{H}} 5.53[\mathrm{~d}, J 8.1, \mathrm{C}(5) H \mathrm{Ph}]$. Purification by column chromatography using hexane-ethyl acetate (gradient elution 10-20\% ethyl acetate) gave 85 as a white solid (0.05 g, 20\%), mp 187-188 ${ }^{\circ} \mathrm{C}$; (Found C, 70.99; H, 5.04; N, 11.27; S, 8.10. $\mathrm{C}_{23} \mathrm{H}_{19} \mathrm{~N}_{3} \mathrm{OS}$ requires $\mathrm{C}, 71.66 ; \mathrm{H}, 4.97 ; \mathrm{N}, 10.90 ; \mathrm{S}, 8.32 \%) ; v_{\max } / \mathrm{cm}^{-1}(\mathrm{KBr}) 3392(\mathrm{NH}), 3189(\mathrm{NH})$, $2918(\mathrm{CH}), 1661(\mathrm{CO}), 1602,1523 ; \delta_{\mathrm{H}}\left(300 \mathrm{MHz}, \mathrm{CDCl}_{3}\right) 2.32\left(3 \mathrm{H}, \mathrm{s}, \mathrm{ArCH}_{3}\right), 7.10-$ $7.30(7 \mathrm{H}, \mathrm{m}, \mathrm{ArH}), 7.36-7.45$ (5H, m, ArH), 7.82 (2H, dd, J 7.8, 1.8, ArH), 9.75 (1H, br s, $\mathrm{N} H$ of carboxamide); $\delta_{\mathrm{C}}\left(75.5 \mathrm{MHz}, \mathrm{CDCl}_{3}\right) 20.9\left(\mathrm{CH}_{3}, \mathrm{ArCH}_{3}\right), 103.1[\mathrm{C}, C(4)]$, $120.3,126.3,126.5,128.1,128.5,128.9,129.6(7 \times \mathrm{CH}, 7 \times$ aromatic $\mathrm{CH}, 7$ signals for 8 carbons), 134.5, 134.8, $135.6(3 \times \mathrm{C}, 3 \times$ aromatic $C), 141.2[\mathrm{C}, \mathrm{br}, C(3)$ or $C(5)], 156.6$ $(\mathrm{C}, \mathrm{CO})$. The signal for either $\mathrm{C}(3)$ or $\mathrm{C}(5)$ was not detected; HRMS (ES+): Exact mass calculated for $\mathrm{C}_{23} \mathrm{H}_{20} \mathrm{~N}_{3} \mathrm{OS}[\mathrm{M}+\mathrm{H}]^{+}$, 386.1327. Found 386.1338; m/z (ES+) 771.2 $\left\{\left[\left(\mathrm{C}_{46} \mathrm{H}_{38} \mathrm{~N}_{6} \mathrm{O}_{2} \mathrm{~S}_{2}\right)+\mathrm{H}^{+}\right], 26 \%\right\}, 386.0\left\{\left[\left(\mathrm{C}_{23} \mathrm{H}_{19} \mathrm{~N}_{3} \mathrm{OS}\right)+\mathrm{H}^{+}\right], 100 \%\right\}$. 
Reference List

1. J. A. Moore, W. F. Holton, E. L. Wittle, J.Am.Chem.Soc., 1962, 84, 390-395.

2. D. E. McGreer, I. M. E. Masters, M. T. H. Liu, J.Chem.Soc., Perkin Trans.2, 1975, 1791-1794.

3. M. Kissane, D. Lynch, J. Chopra, S. E. Lawrence, A. R. Maguire, Tetrahedron: Asymmetry, 2008, 19, 1256-1273.

4. L. G. Tensmeyer, C. Ainsworth, J.Org.Chem., 1966, 31, 1878-1883.

5. J. L. Huppatz, J. N. Phillips, B. Witrzens, Agric.Biol.Chem., 1984, 48, 45-50.

6. T. van Herk, J. Brussee, A. M. C. H. van den Nieuwendijk, P. A. M. van der Klein, A. P. Ijzerman, C. Stannek, A. Burmeister, A. Lorenzen, J.Med.Chem., 2003, 46, 3945-3951. 